\title{
Periodically Driven Quantum Systems: Effective Hamiltonians and Engineered Gauge Fields
}

\author{
N. Goldman ${ }^{1,2, *}$ and J. Dalibard ${ }^{1,2, \dagger}$ \\ ${ }^{1}$ Collège de France, 11, place Marcelin Berthelot, 75005 Paris, France \\ ${ }^{2}$ Laboratoire Kastler Brossel, CNRS, UPMC, ENS, 24 rue Lhomond, 75005 Paris, France
}

(Received 16 April 2014; published 18 August 2014)

\begin{abstract}
Driving a quantum system periodically in time can profoundly alter its long-time dynamics and trigger topological order. Such schemes are particularly promising for generating nontrivial energy bands and gauge structures in quantum-matter systems. Here, we develop a general formalism that captures the essential features ruling the dynamics: the effective Hamiltonian, but also the effects related to the initial phase of the modulation and the micromotion. This framework allows for the identification of driving schemes, based on general $N$-step modulations, which lead to configurations relevant for quantum simulation. In particular, we explore methods to generate synthetic spin-orbit couplings and magnetic fields in cold-atom setups.
\end{abstract}

DOI: 10.1103/PhysRevX.4.031027

\section{INTRODUCTION}

Realizing novel states of matter using controllable quantum systems constitutes a common interest that connects various fields of condensed-matter physics. Two main routes are currently investigated to reach this goal. The first method consists in fabricating materials [1,2], or artificial materials [3-10], which present intrinsic effects that potentially give rise to interesting phases of matter. For instance, one can create topological insulating materials, which present large intrinsic spin-orbit couplings [1]. The second method, which is now commonly considered in the field of quantum simulation, consists in driving a system using external fields [11-13] or mechanical deformations $[4,14]$ to generate synthetic, or effective, gauge structures. Formally, these driven-induced gauge fields enter an effective Hamiltonian that captures the essential characteristics of the modulated system. This strategy exploits the fact that modulation schemes can be tailored in such a way that effective Hamiltonians reproduce the Hamiltonians of interesting static systems. Furthermore, the versatility of driving schemes might enable one to explore situations that remain unreachable in static fabricated systems.

In this context, several works have proposed methods to engineer effective magnetic fields or spin-orbit coupling based on driven cold-atom or ion-trap systems [15-29]. Recently, these proposals led to the realization of the

\footnotetext{
*nathan.goldman@lkb.ens.fr jean.dalibard@lkb.ens.fr
}

Published by the American Physical Society under the terms of the Creative Commons Attribution 3.0 License. Further distribution of this work must maintain attribution to the author(s) and the published article's title, journal citation, and DOI.
Subject Areas: Atomic and Molecular Physics

Hofstadter model (i.e., a lattice system penetrated by a uniform magnetic field [30]), using "shaken" optical lattices [31-34], and to frustrated magnetism, using triangular optical lattices $[35,36]$. Modulating a honeycomb optical lattice [37] also led to the experimental realization of the Haldane model [38]. Besides, artificial magnetic fields have been created in strained graphene [14]. Another field of research focuses on the possibility to create "Floquet topological insulating states" by subjecting trivial insulators or semimetals (e.g., semiconductors or graphene) to external electromagnetic radiation [39-46]. This strategy has been generalized for superfluids, where "Floquet Majorana fermions" could be created by driving superconducting systems [47-51]. The topological invariants (i.e., winding numbers) and the edge-state structures proper to driven systems were analyzed in Refs. [52-58]. Finally, we note that time-periodic modulations can also be simulated in photonics crystals, where time is replaced by a spatial direction [6]; such photonics systems have been fabricated recently [8] with a view to observing the anomalous quantum Hall effect [38], through the imaging of the related topological edge states.

In this work, we develop and explore a general framework that describes periodically driven quantum systems and that generalizes the formalism introduced by Rahav et al. in Ref. [59]. In contrast with the standard Floquet analysis $[60,61]$ or the effective Hamiltonian method presented by Avan et al. in Ref. [62], the present method clearly isolates and identifies the three main characteristics of modulated systems: (1) the effective Hamiltonian underlying the longtime dynamics, (2) the micromotion, and (3) the effects associated with the initial phase of the modulation. These distinct effects will be largely illustrated in this work, based on different examples relevant for the quantum simulation of 
gauge structures, e.g., magnetic fields and spin-orbit couplings. Moreover, this work provides general formulas and methods that can be easily exploited to identify wide families of promising driving schemes.

Before presenting the outline of the paper (Sec. I C), we briefly summarize some important notions related to driven quantum systems, based on basic illustrative examples.

\section{A. Effective Hamiltonians and the micromotion: Two simple illustrations}

We start the discussion by presenting two very simple situations that illustrate in a minimal manner the basic notions and effects encountered in the following.

\section{The Paul trap}

This first illustrative and basic example consists in a particle moving in a modulated harmonic trap [63]. The Hamiltonian is taken in the form

$$
\hat{H}(t)=\hat{H}_{0}+\hat{V} \cos (\omega t)=\frac{\hat{p}^{2}}{2 m}+\frac{1}{2} m \omega_{0}^{2} \hat{x}^{2} \cos (\omega t),
$$

where $\omega=2 \pi / T$ (respectively, $\omega_{0}$ ) denotes the modulation (respectively, harmonic-trap) frequency. The evolution operator after one period of the modulation is evaluated in Appendix A, and it reads

$$
\hat{U}(T)=\exp \left(-i T \hat{H}_{\mathrm{eff}}\right), \quad \hat{H}_{\mathrm{eff}} \approx \hat{H}_{0}+\frac{1}{2} m \Omega^{2} \hat{x}^{2},
$$

expressing the fact that the particle effectively moves in a harmonic trap with frequency $\Omega=\omega_{0}^{2} / \sqrt{2} \omega$. An additional insight is provided by a classical treatment, in which one partitions the motion $x(t)=\bar{x}(t)+\xi(t)$ into a slow and a fast (micromotion) component. As shown in Appendix A, this analysis shows that the effective harmonic potential with frequency $\Omega$ that rules the slow motion $\bar{x}(t)$ is equal to the average kinetic energy associated with the micromotion:

$$
\frac{1}{2} m \Omega^{2} \bar{x}^{2}=\frac{1}{2} m\left\langle\dot{\xi}^{2}\right\rangle,
$$

where the angled brackets denote the average over one period. This classical result illustrates the important role played by the micromotion in modulated systems.

\section{The modulated optical lattice}

As a second example, we consider a modulated 1D lattice, treated in the single-band tight-binding approximation [64-68]. The Hamiltonian is taken in the form (see Appendix B)

$$
\hat{H}(t)=\hat{H}_{0}+\kappa \cos (\omega t) \hat{V}, \quad \hat{V}=\sum_{j} j \hat{a}_{j}^{\dagger} \hat{a}_{j}=\hat{x},
$$

where $\hat{H}_{0}$ describes the nearest-neighbor hopping on the lattice, the operator $\hat{a}_{j}^{\dagger}$ creates a particle at lattice site $x=j a$, and $a$ is the lattice spacing. The effective Hamiltonian describing the slow motion of a particle moving on the modulated lattice can be derived exactly (see Appendix B), yielding the well-known renormalization of the hopping rate by a Bessel function of the first kind

$$
\hat{H}_{\text {eff }}=\mathcal{J}_{0}(\kappa / \omega) \hat{H}_{0} \approx\left(1-\frac{\kappa^{2}}{4 \omega^{2}}+\cdots\right) \hat{H}_{0} .
$$

This effect has been observed experimentally with cold atoms in optical lattices [65,67].

The micromotion also plays an important role in this second example, where it is associated with large oscillations in quasimomentum space. Indeed, within the singleband approximation, a significant modification of the tunneling rate is found when the micromotion oscillation is comparable to the width of the Brillouin zone.

We point out that the Paul trap and the modulated lattice share similar structures (see also Appendixes A and B): Both systems are driven by a modulation of the form $\hat{H}_{0}+\hat{V} \cos (\omega t)$, and their effective Hamiltonians both contain a nontrivial term that is second order in the period $T$. In the present case of the modulated lattice, the term proportional to $(\kappa / \omega)^{2}$ is the first nontrivial term of an infinite series, which can be truncated for $\kappa / \omega<1$; see Eq. (5).

\section{B. The two-step modulation and the ambiguity inherent to the Trotter approach}

Motivated by the two simple examples described above, we consider a general quantum system described by a static Hamiltonian $\hat{H}_{0}$, which is periodically driven by a repeated two-step sequence of the form

$$
\gamma:\left\{\hat{H}_{0}+\hat{V}, \hat{H}_{0}-\hat{V}\right\},
$$

where $\hat{V}$ is some operator. For simplicity, we suppose that the duration of each step is $T / 2$, where $T=2 \pi / \omega$ is the period of the driving sequence $\gamma$. Thus, the square-wave sequence $\gamma$ is qualitatively equivalent to the smooth driving $\hat{H}_{0}+\hat{V} \cos (\omega t)$ encountered in the two examples discussed above.

In the following, the energy $\hbar \omega$ will be considered to be very large compared to all the energies present in the problem, justifying a perturbative treatment in $(1 / \omega)$. The small dimensionless quantity associated with this expansion $\Omega_{\text {eff }} / \omega$ will be made explicit for the various physical problems encountered in the following sections. Typically, $\Omega_{\text {eff }}$ will be identified with the cyclotron frequency in the case of synthetic magnetism (see Sec. VI) or with the spinorbit-coupling strength (see Sec. VII); see also Sec. VIII A.

Starting in an initial state $\left|\psi_{0}\right\rangle$ at time $t_{i}=0$, the state at time $t=N T(N \in \mathbb{N})$ is obtained through the evolution operator 


$$
\begin{aligned}
\hat{U}(t=N T)\left|\psi_{0}\right\rangle & =\left(e^{-i T\left(\hat{H}_{0}-\hat{V}\right) / 2} e^{-i T\left(\hat{H}_{0}+\hat{V}\right) / 2}\right)^{N}\left|\psi_{0}\right\rangle, \\
& =e^{-i N T \hat{H}_{\mathrm{eff}}^{T}}\left|\psi_{0}\right\rangle,
\end{aligned}
$$

where we introduce a time-independent effective Hamiltonian

$$
\hat{U}(T)=e^{-i T \hat{H}_{\mathrm{eff}}^{T}}=e^{-i T\left(\hat{H}_{0}-\hat{V}\right) / 2} e^{-i T\left(\hat{H}_{0}+\hat{V}\right) / 2} .
$$

The product of two exponentials can be simplified through the Baker-Campbell-Hausdorff $(\mathrm{BCH})$ formula, hereafter referred to as the Trotter expansion:

$$
e^{X} e^{Y}=\exp \left(X+Y+\frac{1}{2}[X, Y]+\frac{1}{12}[X-Y,[X, Y]]+\cdots\right),
$$

yielding a simple expression for the effective "Trotter" Hamiltonian

$$
\hat{H}_{\mathrm{eff}}^{\mathcal{T}}=\hat{H}_{0}-i \frac{T}{4}\left[\hat{H}_{0}, \hat{V}\right]+\mathcal{O}\left(T^{2}\right) .
$$

Importantly, the sign in front of the first-order term depends on the starting pulse $\left(\hat{H}_{0}+\hat{V}\right.$ or $\left.\hat{H}_{0}-\hat{V}\right)$ of the driving sequence (6) or, equivalently, on the definition of the starting time $t_{i}$ : Indeed, shifting the starting time $t_{i} \rightarrow$ $t_{i}+(T / 2)$ leads to the opposite term $+i(T / 4)\left[\hat{H}_{0}, \hat{V}\right]$. Thus, the first-order term arising from the Trotter expansion is sensitive to the initial phase $\omega t_{i}$ of the driving. We emphasize that the sign ambiguity is different from the phase of the micromotion sampling, which will be illustrated later in this work. Furthermore, we note that the firstorder term in Eq. (10) can be eliminated by a unitary transformation

$$
\hat{H}_{\mathrm{eff}}^{\mathcal{T}}=\hat{S}^{\dagger} \hat{H}_{0} \hat{S}+\mathcal{O}\left(T^{2}\right), \quad \hat{S}=e^{-i T \hat{V} / 4},
$$

indicating its trivial role in the effective Hamiltonian. Indeed, the long-time behavior of the system described by Eq. (7) can be expressed as

$$
\begin{aligned}
\hat{U}(t=N T)\left|\psi_{0}\right\rangle & =e^{-i N T \hat{H}_{\mathrm{eff}}^{T}}\left|\psi_{0}\right\rangle \\
& =\hat{S}^{\dagger}\left(e^{-i N T \hat{H}_{0}}\right) \hat{S}\left|\psi_{0}\right\rangle+\mathcal{O}\left(T^{2}\right),
\end{aligned}
$$

which indicates that the system first undergoes an initial kick $\hat{S}=e^{-(i T / 4) V}$ (when the sequence $\gamma$ is applied in this order), then evolves "freely" for a long time $t=N T$, and finally undergoes a final sudden kick (i.e., a micromotion). From Eq. (11), we conclude that the first-order term in Eq. (10) cannot be exploited to modify the band structure of $\hat{H}_{0}$ or, equivalently, to generate nontrivial gauge structures (e.g., effective magnetic fields or spin-orbit couplings). This observation is in agreement with the effective Hamiltonians obtained for the Paul trap (2) and the modulated lattice (5), where the first nontrivial terms are found to be second order in the period $T$; see Secs. I A 1 and I A 2.

\section{Outline of the paper}

The rest of the main article is structured as follows. Section II presents the general formalism used to treat timedependent Hamiltonians. The method is then applied to the simple two-step modulation introduced in Eq. (6). Section III illustrates the impact of the initial phase of the modulation on long-time dynamics, based on a simple example. This section highlights the importance of the "kick" operator $\hat{K}(t)$ introduced in Sec. II. Section IV derives useful formulas for the effective Hamiltonian and kick operators in the general case of $N$-step modulations $(N \in \mathbb{Z})$. Section $\mathrm{V}$ explores two specific classes of modulations, characterized by $N=4$ different steps. Section VI applies the latter results to a modulation generating an effective magnetic field in two-dimensional systems. This sequence is explored both in the absence and in the presence of a lattice. Section VII proposes and explores several driving sequences realizing effective spinorbit couplings in two-dimensional spin-1/2 systems. These sequences are also analyzed in the absence and in the presence of a lattice. Section VIII is dedicated to general discussions and conclusions. This final part analyzes the convergence of the perturbative approach introduced in Sec. II. It also briefly discusses the possibility to launch the modulation adiabatically. Finally, we present concluding remarks and outlooks.

\section{THE NOTION OF EFFECTIVE HAMILTONIANS: USING A RELIABLE APPROACH}

\section{A. The formalism}

Having identified the subtleties proper to the analysis based on the BCH Trotter formula in Sec. IB, we now consider an alternative approach inspired by Ref. [59]. Let us first rephrase the general problem based on our previous analysis. We act on an initial state $\left|\psi_{0}\right\rangle$ with a time-periodic Hamiltonian

$$
\begin{gathered}
\hat{H}(t)=\hat{H}_{0}+\hat{V}(t), \\
\hat{V}(t)=\sum_{j=1}^{\infty} \hat{V}^{(j)} e^{i j \omega t}+\hat{V}^{(-j)} e^{-i j \omega t},
\end{gathered}
$$

between times $t_{i}$ and $t_{f}$, the period of the driving being $T=2 \pi / \omega$. In Eq. (14), we explicitly Fourier expand the time-dependent potential, to take higher harmonics into account. There are three distinct notions: (1) the initial phase of the Hamiltonian at time $t_{i}$ (i.e., $\omega t_{i} \bmod 2 \pi$ ): The way the driving starts, namely, $\hat{V}\left(t_{i}\right)$, may have an important impact on the dynamics; (2) the evolution of the system between the interval $\Delta t=t_{f}-t_{i}$, which can be 
arbitrarily long, and during which the Hamiltonian $\hat{H}(t)$ is applied; and (3) the final phase of the Hamiltonian at time $t_{f}$ (i.e., $\omega t_{f}$ modulo $2 \pi$ ): This final step describes the micromotion. These concepts were illustrated in Eq. (12) for the simple two-step sequence (6) presented in Sec. I B. In order to separate these three effects in a clear manner, we generalize the approach of Ref. [59] and reexpress the evolution operator as

$$
\begin{aligned}
\hat{U}\left(t_{i} \rightarrow t_{f}\right) & =\hat{\mathcal{U}}^{\dagger}\left(t_{f}\right) e^{-i\left(t_{f}-t_{i}\right) \hat{H}_{\mathrm{eff}} \hat{\mathcal{U}}\left(t_{i}\right),} \\
& =e^{-i \hat{K}\left(t_{f}\right)} e^{-i\left(t_{f}-t_{i}\right) \hat{H}_{\mathrm{eff}}} e^{i \hat{K}\left(t_{i}\right),}
\end{aligned}
$$

where we impose that (i) $\hat{H}_{\text {eff }}$ is a time-independent operator, (ii) $\hat{K}(t)$ is a time-periodic operator $\hat{K}(t+T)=$ $\hat{K}(t)$ with zero average over one period, and (iii) $\hat{H}_{\text {eff }}$ does not depend on the starting time $t_{i}$, which can be realized by transferring all undesired terms into the kick operator $\hat{K}\left(t_{i}\right)$. Similarly, $\hat{H}_{\text {eff }}$ does not depend on the final time $t_{f}$.

Following a perturbative expansion in powers of $(1 / \omega)$, we obtain (see Appendix C)

$$
\begin{aligned}
\hat{H}_{\text {eff }}= & \hat{H}_{0}+\frac{1}{\omega} \sum_{j=1}^{\infty} \frac{1}{j}\left[\hat{V}^{(j)}, \hat{V}^{(-j)}\right] \\
& +\frac{1}{2 \omega^{2}} \sum_{j=1}^{\infty} \frac{1}{j^{2}}\left(\left[\left[\hat{V}^{(j)}, \hat{H}_{0}\right], \hat{V}^{(-j)}\right]+\left[\left[\hat{V}^{(-j)}, \hat{H}_{0}\right], \hat{V}^{(j)}\right]\right) \\
& +\mathcal{O}\left(T^{3}\right) \\
\hat{K}(t)= & \int^{t} \hat{V}(\tau) d \tau+\mathcal{O}\left(T^{2}\right) \\
= & \sum_{j \neq 0} \frac{1}{i j \omega} \hat{V}^{(j)} e^{i j \omega t}+\mathcal{O}\left(T^{2}\right) .
\end{aligned}
$$

In Eq. (16), we have omitted the second-order terms that mix different harmonics, noting that these terms do not contribute in the situations presented in this work; the complete second-order terms contained in $\hat{H}_{\text {eff }}$ and $\hat{K}(t)$ are presented in Appendix C; see Eqs. (C10) and (C11). By construction, and in contrast to the Trotter approach, the expressions (16) and (17) constitute a strong basis to evaluate the relevance of periodic driving schemes in view of realizing nontrivial and robust effects, such as nonzero effective magnetic fields.

We conclude this section by pointing out that effective Hamiltonians can also be obtained through Floquet theory [60]. However, as is apparent in Refs. [60,61], there is a priori no natural constraint within Floquet theory that prevents the "Floquet" effective Hamiltonians to contain $t_{i}$-dependent terms. A possible way to get rid of these terms in Floquet theory is to consider an adiabatic launching of the driving [66,67], such that the evolving state is constrained to remain in the same (principal) quasienergy multiplicity at all times (the multiplicity being well separated by the large energy $\hbar \omega$ ). However, for the sake of generality and clarity, we will follow here the approach based on the partitionment (15) discussed in this section, which provides an unambiguous and physically relevant definition for the effective Hamiltonian. Finally, we point out that the convergence of the perturbative expansion in powers of $(1 / \omega)$ and leading to Eq. (16) is by no means guaranteed, as will be discussed later in Sec. VIII A.

\section{B. Illustration of the formalism: Back to the two-step sequence}

As a first illustration, let us apply the expressions (16) and (17) to the simple two-step sequence in Eq. (6). The Hamiltonian is given by $\hat{H}(t)=\hat{H}_{0}+f(t) \hat{V}$, where $f(t)$ is the standard square-wave function. Expanding $f(t)$ into its Fourier components, we obtain a simple expression for the $\hat{V}^{(j)}$ operators introduced in Eqs. (13) and (14). A direct evaluation of Eqs. (16) and (17) then implies (see Appendix D)

$$
\begin{gathered}
\hat{H}_{\text {eff }}=\hat{H}_{0}+\frac{\pi^{2}}{24 \omega^{2}}\left[\left[\hat{V}, \hat{H}_{0}\right], \hat{V}\right]+\mathcal{O}\left(1 / \omega^{3}\right), \\
\hat{K}(t)=-\frac{\pi}{2 \omega} \hat{V}+|t| \hat{V}+\mathcal{O}\left(1 / \omega^{2}\right) \text { for } t \in\left[-\frac{T}{2}, \frac{T}{2}\right], \\
\text { such that the evolution operator is given by [Eq. (15)] } \\
\hat{U}(0 \rightarrow t)=e^{-i \hat{K}(t)} e^{-i t\left[\hat{H}_{0}+\mathcal{O}\left(1 / \omega^{2}\right)\right]} e^{-i T \hat{V} / 4}
\end{gathered}
$$

in agreement with Eq. (12). Note that the amplitude of the initial kick $\hat{K}\left(t_{i}\right)$ is maximal at the initial time $t_{i}=0$ and that it is 0 at time $t_{i}=T / 4$. In contrast with the Trotter analysis, the approach based on the partitionment (15) directly identifies (a) the absence of a first-order term in the effective Hamiltonian $\hat{H}_{\text {eff }}$, (b) the initial kick produced by the operator $\hat{S}=\exp [i \hat{K}(0)]=\exp (-i T \hat{V} / 4)$, and (c) the micromotion $\exp [-i \hat{K}(t)]$. We note that since the latter operator satisfies $\exp [-i \hat{K}(N T)]=\exp [(-i \hat{K}(0)]$ $=\exp (i T \hat{V} / 4)=\hat{S}^{\dagger}$, we exactly recover Eq. (12) for $t=N T$.

The result in Eq. (18), which is associated with the two-step sequence (6), is to be compared with the smooth driving considered in Secs. I A 1 and I A 2:

$$
\hat{H}(t)=\hat{H}_{0}+\hat{V}(t)=\hat{H}_{0}+\hat{V} \cos (\omega t),
$$

which is readily treated using Eq. (16). Setting $V^{(1)}=$ $V^{(-1)}=\hat{V} / 2$ yields

$$
\begin{gathered}
\hat{H}_{\mathrm{eff}}=\hat{H}_{0}+\frac{1}{4 \omega^{2}}\left[\left[\hat{V}, \hat{H}_{0}\right], \hat{V}\right]+\mathcal{O}\left(1 / \omega^{3}\right), \\
\hat{K}(t)=[\sin (\omega t) / \omega] \hat{V}+\mathcal{O}\left(1 / \omega^{2}\right),
\end{gathered}
$$


which is indeed qualitatively equivalent to Eqs. and (19).

We now apply the formalism to the two examples presented in Secs. I A 1 and I A 2.

(a) The Paul trap.-We readily recover the effective Hamiltonian in Eq. (2) by inserting the operators defined in Eq. (1) into Eq. (21). Furthermore, Eq. (22) provides an approximate expression for the micromotion underlying the slow motion in the Paul trap. (See also Appendix A for more details.)

(b) The modulated lattice.-Inserting the operators defined in Eq. (4) into Eqs. (21) and (22) yields

$$
\begin{gathered}
\hat{H}_{\text {eff }}=\left(1-\frac{\kappa^{2}}{4 \omega^{2}}\right) \hat{H}_{0}+\mathcal{O}\left(1 / \omega^{3}\right), \\
\hat{K}(t)=(\kappa / \omega) \hat{x} \sin (\omega t)+\mathcal{O}\left(1 / \omega^{2}\right),
\end{gathered}
$$

where we indeed recover the first terms of the Bessel function expansion in Eq. (5). We note that the maximal amplitude of the kick associated with the micromotion is given by $\exp [-i \hat{K}(T / 4)]=\exp [-i(\kappa / \omega) \hat{x}]$, which corresponds to a translation in the Brillouin zone by an amount $\Delta k=\kappa / \omega$. We thus recover the fact that the modification of the hopping rate becomes appreciable when the micromotion is comparable to the width of the Brillouin zone $\Delta k \approx \pi$ : Indeed, $\mathcal{J}_{0}[(\kappa / \omega)=\pi] \approx-1 / 3$ is very close to the minimal value of the Bessel function and thus corresponds to a dramatic change in the tunneling rate. [The maximal value of the Bessel function is $\mathcal{J}_{0}(0)=1$, which corresponds to the standard hopping rate in the absence of shaking.]

The result in Eq. (23) stems from the perturbative expansion in powers of $(1 / \omega)$. However, the formalism presented in Sec. II A also allows for an exact treatment of the modulated-lattice problem. The full derivation is given in Appendix E, where we recover the Bessel-renormalizedhopping result of Eq. (5). Moreover, this derivation provides an exact form for the kick operator; see Eq. (E10). The latter expression indicates that the maximal amplitude of the kick operator is given by $\hat{K}=\hat{x} \kappa / \omega$, which is precisely the result discussed above based on the perturbative treatment.

\section{LAUNCHING THE DRIVING: ILLUSTRATION OF THE INITIAL KICK}

In the last section, we introduced the partitionment of the evolution operator

$$
\hat{U}\left(t_{i} \rightarrow t\right)=e^{-i \hat{K}(t)} e^{-i\left(t-t_{i}\right) \hat{H}_{\text {eff }}} e^{i \hat{K}\left(t_{i}\right)},
$$

which highlights the fact that the system undergoes an initial kick $\exp \left[i \hat{K}\left(t_{i}\right)\right]$ before evolving according to the (time-independent) effective Hamiltonian. This initial kick depends on the launching time of the sequence $t_{i}$, through
Eq. (17), and it can have a great impact on long-time dynamics. It is the aim of this section to illustrate this effect, based on a basic but enlightening example.

Consider a particle driven by a uniform force that alternates its sign in a pulsed manner: $+F,-F, \ldots$. This system obeys the two-step sequence in Eq. (6) with $\hat{H}_{0}=\hat{p}^{2} / 2 m$ and $\hat{V}=-F \hat{x}$. The effective Hamiltonian and kick operators are readily obtained through Eqs. (18) and (19), yielding

$$
\hat{H}_{\text {eff }}=\hat{p}^{2} / 2 m+\text { cst, }
$$

$$
\hat{K}(t)=(F T / 4) \hat{x}-|t| F \hat{x}+\mathcal{O}\left(1 / \omega^{2}\right) \quad \text { for } t \in\left[-\frac{T}{2}, \frac{T}{2}\right] .
$$

The initial-kick operator is $\hat{K}\left(t_{i}\right)=\hat{x} F T / 4$ for $t_{i}=0$, and $\hat{K}\left(t_{i}\right)=-\hat{x} F T / 4$ for $t_{i}=T / 2$, and its effect is thus to modify the initial mean velocity $v\left(t_{i}\right) \rightarrow v\left(t_{i}\right) \pm F T / 4 m$ before the long-time free evolution. We emphasize that the initial-kick operator is 0 when starting the sequence at time $t_{i}= \pm T / 4$.

Further insight is provided by exactly computing the evolution operator, at time $t=T$ :

$$
\begin{aligned}
\hat{U}(T) & =\exp \left[-i T\left(\frac{1}{2 m}\left[\hat{p}+\mathcal{A}\left(t_{i}\right)\right]^{2}+\mathrm{cst}\right)\right] \\
& =\exp \left[-i T \tilde{H}_{\mathrm{eff}}\left(t_{i}\right)\right],
\end{aligned}
$$

where the gauge potential is given by $\mathcal{A}\left(t_{i}\right)= \pm F T / 4$, whether the starting time is $t_{i}=0$ or $t_{i}=T / 2$, respectively. We note that the effective Hamiltonian $\tilde{H}_{\text {eff }}\left(t_{i}\right)$ is related to the $t_{i}$-independent effective Hamiltonian $\hat{H}_{\text {eff }}$ in Eq. (25) through the unitary (gauge) transformation involving the kick operator $\exp [i \hat{K}(0)]=\exp [i \mathcal{A} \hat{x}]$, in agreement with Eq. (24).

Treating the basic mechanics problem associated with $\tilde{H}_{\text {eff }}\left(t_{i}\right)$ semiclassically, we recover that the initial kick modifies the initial velocity $v\left(t_{i}\right) \rightarrow v\left(t_{i}\right)+\mathcal{A} / m$. Hence, for an arbitrary $v\left(t_{i}\right)$, the position of the particle $x(t \gg T)$ will significantly depend on whether the pulse sequence has started with a pulse $+\hat{V}\left[t_{i}=0\right]$ or $-\hat{V}\left[t_{i}=T / 2\right]$ : The dynamics is strongly sensitive to the initial phase of the modulation. In Fig. 1, we compare these predictions to the real (classical) dynamics of the pulse-driven particle. This figure illustrates the sensitivity to the initial phase of the driving but also the effects of micromotion present at all times for all configurations.

In general, the effects related to the initial phase of the modulation may remain important in more sophisticated driven systems. The sensitivity to the initial phase will be further illustrated in the context of driven-induced Rashba spin-orbit couplings in Sec. VII (see Fig. 5). 


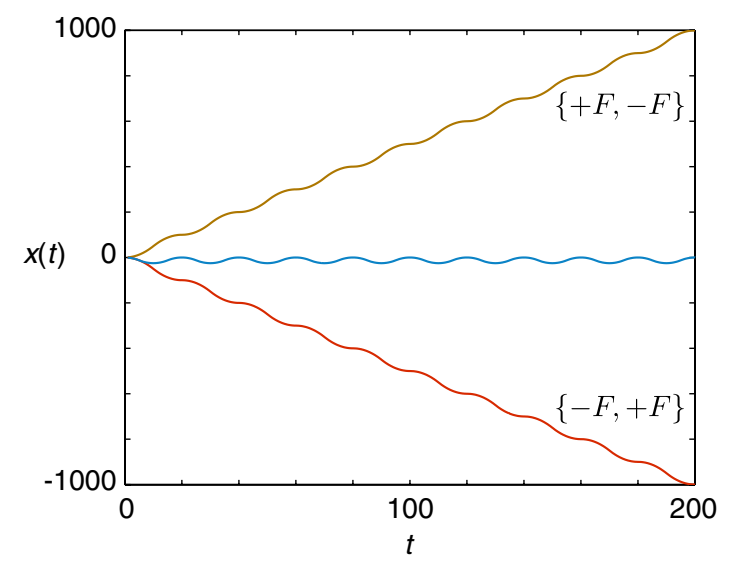

FIG. 1. Sensitivity to the initial phase of the driving. The brown curve represents the classical dynamics of a particle driven by a repeating pulse sequence $\{+F,-F\}$, where $F>0$ is a uniform force; the period is $T=20$, and the particle is initially at rest $v\left(t_{i}\right)=$ 0 at $x\left(t_{i}\right)=0$. The red curve is the same but considers the sequence $\{-F,+F\}$, i.e., shifts the starting time $t_{i} \rightarrow t_{i}+T / 2$. The blue curve is also the same but starts the sequence with $-F$ during a time $T / 4$ and then repeats the sequence $\{+F,-F\}$, i.e., shifts the starting time $t_{i} \rightarrow t_{i}-T / 4$. Note that the initial kick $\hat{K}\left(t_{i}\right)$ is inhibited in the latter case, while it is opposite in the two former cases [Eq. (26)]. In all the plots, the particle depicts a small micromotion, captured by $\hat{K}(t)$ in Eq. (26). The brown and red curves highlight the great sensitivity to the initial phase of the modulation.

\section{MULTISTEP SEQUENCES}

Going beyond the two-step driving sequence in Eq. (6) potentially increases the possibility to engineer interesting effective Hamiltonians and gauge structures. We now derive the effective Hamiltonian for the general situation where the pulse sequence is characterized by the repeated $N$-step sequence

$$
\gamma_{N}=\left\{\hat{H}_{0}+\hat{V}_{1}, \hat{H}_{0}+\hat{V}_{2}, \hat{H}_{0}+\hat{V}_{3}, \ldots, \hat{H}_{0}+\hat{V}_{N}\right\},
$$

where the $\hat{V}_{m}$ 's are arbitrary operators. In the following, we consider that the duration of each step is $\tau=T / N$, where $T$ is the driving period, and we further impose that

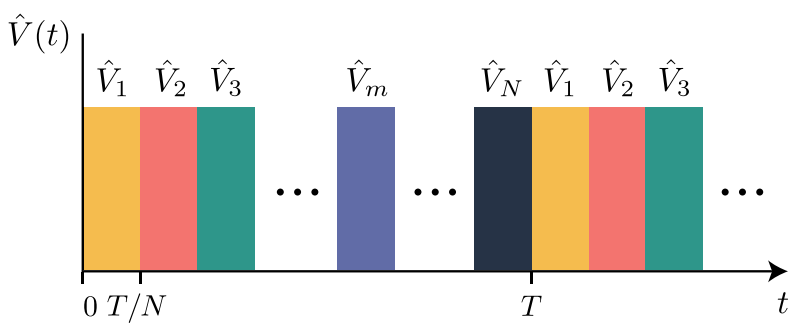

FIG. 2. Schematic representation of the general $N$-step driving sequence $\gamma_{N}$ introduced in Eqs. (27) and (28), where $\hat{H}(t)=$ $\hat{H}_{0}+\hat{V}(t)$.

$\sum_{m=1}^{N} \hat{V}_{m}=0$. The general driving sequence $\gamma_{N}$ is illustrated in Fig. 2. Note that $\gamma_{N}$ reduces to the simple sequence (6) of Sec. IB for $N=2$ and $\hat{V}_{1}=-\hat{V}_{2}=\hat{V}$.

The general Hamiltonian corresponding to the pulse sequence (27) is written as

$$
\begin{aligned}
\hat{H}(t) & =\hat{H}_{0}+\sum_{m=1}^{N} f_{m}(t) \hat{V}_{m} \\
f_{m}(t) & =1 \quad \text { for }(m-1) T / N \leq t \leq m T / N \text { (otherwise } 0) \\
& =\frac{1}{2 \pi i} \sum_{n \neq 0} \frac{1}{n} e^{-i 2 \pi n m / N}\left(e^{i n(2 \pi / N)}-1\right) e^{i n \omega t}
\end{aligned}
$$

where the last line of Eq. (28) provides the Fourier series of the square functions $f_{m}(t)$. In order to apply Eqs. (16) and (17), we expand the Hamiltonian in terms of the harmonics

$$
\begin{gathered}
\hat{H}(t)=\hat{H}_{0}+\sum_{j \neq 0} \hat{V}^{(j)} e^{i j \omega t}, \\
\hat{V}^{(j)}=\frac{1}{2 \pi i} \sum_{m=1}^{N} \frac{1}{j} e^{-i 2 \pi j m / N}\left(e^{i j(2 \pi / N)}-1\right) \hat{V}_{m},
\end{gathered}
$$

where we use the Fourier series in Eq. (28). The effective Hamiltonian and the initial-kick operator $\hat{K}(0)$ are then given by the general expressions (see Appendix F)

$$
\begin{aligned}
\hat{H}_{\mathrm{eff}}=\hat{H}_{0} & +\frac{2 \pi i}{N^{3} \omega} \sum_{m<n=2}^{N} \mathcal{C}_{m, n}\left[\hat{V}_{m}, \hat{V}_{n}\right]+\frac{\pi^{2}(N-1)^{2}}{6 N^{4} \omega^{2}} \sum_{m=1}^{N}\left[\left[\hat{V}_{m}, \hat{H}_{0}\right], \hat{V}_{m}\right] \\
& +\frac{\pi^{2}}{6 N^{4} \omega^{2}} \sum_{m<n=2}^{N} \mathcal{D}_{m, n}\left(\left[\left[\hat{V}_{m}, \hat{H}_{0}\right], \hat{V}_{n}\right]+\left[\left[\hat{V}_{n}, \hat{H}_{0}\right], \hat{V}_{m}\right]\right)+\mathcal{O}\left(1 / \omega^{3}\right) \\
\hat{K}(0)= & \frac{2 \pi}{N^{2} \omega} \sum_{m=1}^{N} \hat{V}_{m} m+\mathcal{O}\left(1 / \omega^{2}\right)
\end{aligned}
$$

where $\mathcal{C}_{m, n}=\frac{N}{2}+m-n$ and $\mathcal{D}_{m, n}=1+N^{2}-6 N(n-m)+6(n-m)^{2}$.

We have again omitted the harmonic-mixing terms given in Eq. (C10), which do not contribute for the sequences considered here. The result in Eq. (31) clearly highlights the fact that the initial kick $\hat{K}(0)$ depends on the way the pulse sequence starts, whereas the effective Hamiltonian $\hat{H}_{\text {eff }}$ is independent of this choice: Shifting the pulse sequence, 
namely, redefining the operators $\hat{V}_{m} \rightarrow \hat{V}_{m+p}$, with $p \in \mathbb{Z}$, results in a change in $\hat{K}(0)$ but leaves $\hat{H}_{\text {eff }}$ invariant.

We have applied the formula (31) to general sequences with $N=3$ and $N=4$ different steps, and we present the associated results in Eqs. (G1) and (G2) in Appendix G. In contrast with the case $N=2$, we find that sequences with $N=3$ or $N=4$ steps can potentially lead to nontrivial effects that are first order in $(1 / \omega)$. We point out that the scheme proposed by Kitagawa et al. [52] to realize the Haldane model using a modulated honeycomb lattice corresponds to the case $N=3$. Moreover, the model of Refs. [54,58], which features topological Floquet edge states, corresponds to the case $N=5$. In the following, we will further explore and illustrate the case $N=4$, with a view to creating synthetic magnetic fields and spin-orbit couplings with cold atoms (see also Refs. $[15,25,26]$ ).

\section{SEQUENCES WITH $N=4$ STEPS}

Motivated by the importance and versatility of four-step sequences to generate nontrivial effective potentials and gauge structures, we now explore two specific examples of such sequences that lead to different effects. The following paragraphs will constitute a useful guide for the applications presented in Secs. VI and VII.

\section{A. The class of sequences $\alpha$}

Let us first consider the following four-step sequence:

$$
\alpha:\left\{\hat{H}_{0}+\hat{A}, \hat{H}_{0}+\hat{B}, \hat{H}_{0}-\hat{A}, \hat{H}_{0}-\hat{B}\right\},
$$

which corresponds to $\gamma_{4}$ in Eq. (27) with $\hat{V}_{1}=-\hat{V}_{3}=\hat{A}$ and $\hat{V}_{2}=-\hat{V}_{4}=\hat{B}$. We now apply the formula (31) [see also Eq. (G2)] and we obtain

$$
\begin{aligned}
\hat{H}_{\mathrm{eff}}= & \hat{H}_{0}+\frac{i \pi}{8 \omega}[\hat{A}, \hat{B}]+\frac{\pi^{2}}{48 \omega^{2}}\left(\left[\left[\hat{A}, \hat{H}_{0}\right], \hat{A}\right]+\left[\left[\hat{B}, \hat{H}_{0}\right], \hat{B}\right]\right) \\
& +\mathcal{O}\left(1 / \omega^{3}\right), \\
\hat{K}(0)= & -\frac{\pi}{4 \omega}(\hat{A}+\hat{B})+\mathcal{O}\left(1 / \omega^{2}\right),
\end{aligned}
$$

where the expression for $\hat{K}(0)$ is obtained for a sequence $\alpha$ starting with the pulse $+\hat{A}$ [and we remind readers that the kick operator $\hat{K}(0)$ depends on this choice]. The result in Eq. (33) shows that the driving schemes belonging to the class $\alpha$ can generate a combination of first-order and second-order terms, which might potentially lead to interesting observable effects; see Secs. VI and VII. In particular, we note that the scheme of Ref. [15] to generate synthetic magnetic flux in optical lattices belongs to this class; see Sec. VI.

Finally, we note that the pulse sequence $\alpha$ can be approximated by the smooth driving $\hat{V}(t)=\hat{A} \cos (\omega t)+$ $\hat{B} \sin (\omega t)$. In this case, a direct evaluation of Eq. (16) yields

$$
\begin{aligned}
\hat{H}_{\mathrm{eff}}= & \hat{H}_{0}+\frac{i}{2 \omega}[\hat{A}, \hat{B}] \\
& +\frac{1}{4 \omega^{2}}\left(\left[\left[\hat{A}, \hat{H}_{0}\right], \hat{A}\right]+\left[\left[\hat{B}, \hat{H}_{0}\right], \hat{B}\right]\right)+\mathcal{O}\left(1 / \omega^{3}\right),
\end{aligned}
$$

which is indeed approximately equal to the effective Hamiltonian (33) associated with the pulsed system.

\section{B. The class of sequences $\beta$}

We now consider an apparently similar four-step sequence

$$
\beta:\left\{\hat{H}_{0}+\hat{A}, \hat{H}_{0}-\hat{A}, \hat{H}_{0}+\hat{B}, \hat{H}_{0}-\hat{B}\right\},
$$

which corresponds to $\gamma_{4}$ in Eq. (27) with $\hat{V}_{1}=-\hat{V}_{2}=\hat{A}$ and $\hat{V}_{3}=-\hat{V}_{4}=\hat{B}$. Applying the formula (31) [Eq. (G2)] yields

$$
\begin{aligned}
\hat{H}_{\mathrm{eff}}= & \hat{H}_{0}+\frac{5 \pi^{2}}{384 \omega^{2}}\left(\left[\left[\hat{A}, \hat{H}_{0}\right], \hat{A}\right]+\left[\left[\hat{B}, \hat{H}_{0}\right], \hat{B}\right]\right) \\
& -\frac{\pi^{2}}{128 \omega^{2}}\left(\left[\left[\hat{A}, \hat{H}_{0}\right], \hat{B}\right]+\left[\left[\hat{B}, \hat{H}_{0}\right], \hat{A}\right]\right)+\mathcal{O}\left(1 / \omega^{3}\right), \\
\hat{K}(0)= & -\frac{\pi}{8 \omega}(\hat{A}+\hat{B})+\mathcal{O}\left(1 / \omega^{2}\right),
\end{aligned}
$$

where $\hat{K}(0)$ corresponds to a sequence $\beta$ starting with the pulse $+\hat{A}$. The result in Eq. (35) emphasizes two major differences between the $\alpha$ and the $\beta$ classes: (a) The effective Hamiltonian associated with the $\beta$ sequence does not contain any first-order term, and in this sense, this $N=4$ sequence resembles the case $N=2$. (b) The $\beta$ sequence generates additional second-order terms that mix the pulsed operators $\hat{A}$ and $\hat{B}$, i.e., terms of the form $\left[\left[\hat{A}, \hat{H}_{0}\right], \hat{B}\right]$ that are not present in the class $\alpha$ [Eq. (33)].

As illustrated in Sec. VII, the schemes of Refs. $[25,26]$ to generate spin-orbit couplings in cold gases can be expressed in the form of sequences $\beta$.

\section{PHYSICAL ILLUSTRATION: GENERATING SYNTHETIC MAGNETIC FIELDS IN COLD GASES}

In this section, we apply the results of Sec. V to generate synthetic magnetic fields in one-component atomic gases, using a four-step sequence of type $\alpha$; see Eq. (32).

\section{A. Without a lattice}

We first consider atoms moving in two-dimensional free space, such that $\hat{H}_{0}=\left(\hat{p}_{x}^{2}+\hat{p}_{y}^{2}\right) / 2 m$. Inspired by Ref. [15], we drive the system with a pulse sequence $\alpha$ [see Eq. (32)] with the operators $\hat{A}=\left(\hat{p}_{x}^{2}-\hat{p}_{y}^{2}\right) / 2 m$ and $\hat{B}=\kappa \hat{x} \hat{y}$. We will comment later on the possibility to implement such a scheme practically. Over a period, the evolution is thus given by the sequence 


$$
\left\{\frac{\hat{p}_{x}^{2}}{m}, \frac{\hat{p}_{x}^{2}+\hat{p}_{y}^{2}}{2 m}+\kappa \hat{x} \hat{y}, \frac{\hat{p}_{y}^{2}}{m}, \frac{\hat{p}_{x}^{2}+\hat{p}_{y}^{2}}{2 m}-\kappa \hat{x} \hat{y}\right\}
$$

which consists in repeatedly allowing for the movement in a pulsed and directional manner while subjecting the cloud to an alternating quadrupolar field. The corresponding effective Hamiltonian is given by Eq. (33), which yields, up to second order $\left(1 / \omega^{2}\right)$,

$$
\begin{aligned}
\hat{H}_{\mathrm{eff}} & =\frac{1}{2 m}\left[\left(\hat{p}_{x}-A_{x}\right)^{2}+\left(\hat{p}_{y}-A_{y}\right)^{2}\right]+\frac{1}{2} m \omega_{h}^{2}\left(\hat{x}^{2}+\hat{y}^{2}\right), \\
\mathcal{A} & =(-m \Omega \hat{y}, m \Omega \hat{x}), \quad \Omega=\frac{\pi \kappa}{8 m \omega}, \quad \omega_{h}=\sqrt{\frac{5}{3}} \Omega,
\end{aligned}
$$

which corresponds to the realization of a perpendicular and uniform synthetic magnetic field

$$
\boldsymbol{B}=2 m \Omega \mathbf{1}_{z}=\frac{\pi \kappa}{4 \omega} \mathbf{1}_{z} .
$$

We point out that the second-order corrections in Eq. (33) lead to a harmonic confinement, which dominates over the centrifugal force generated by the first-order term, resulting in an overall trapping potential in Eq. (37). Defining the cyclotron frequency $\omega_{c}=B / m$, we obtain the ratio between the confinement and cyclotron frequencies

$$
\omega_{h} / \omega_{c}=(1 / 2) \sqrt{5 / 3} \approx 0.64 .
$$

The induced confinement is a special feature of the driving scheme (36). It has a significant impact on the dynamics, which illustrates the fact that the perturbative expansion in $(1 / \omega)$ should not be limited, in typical applications, to its first-order terms. (See Sec. VIII A for a more detailed discussion.)

\section{B. With optical lattices}

A similar scheme can be applied to cold atoms in optical lattices, where a uniform synthetic magnetic field would provide a platform to simulate the Hofstadter model $[30,32,33,69,70]$. Here, we suppose that the atoms evolve within a two-dimensional optical square lattice and that their dynamics is well captured by a single-band tight-binding description. The static Hamiltonian is thus taken in the form

$$
\begin{aligned}
\hat{H}_{0} & =-J \sum_{m, n} \hat{a}_{m+1, n}^{\dagger} \hat{a}_{m, n}+\hat{a}_{m, n+1}^{\dagger} \hat{a}_{m, n}+\text { H.c. } \\
& =\bar{p}_{x}^{2} / 2 m^{*}+\bar{p}_{y}^{2} / 2 m^{*}
\end{aligned}
$$

where $J$ is the hopping amplitude, $\hat{a}_{m, n}^{\dagger}$ creates a particle at lattice site $\boldsymbol{x}=(m a, n a)$, and $a$ is the lattice spacing. In Eq. (41), we introduce the notation $\bar{p}_{x, y}^{2} / 2 m^{*}$ to denote hopping along the $(x, y)$ directions and also the effective mass $m^{*}=1 /\left(2 \mathrm{Ja}^{2}\right)$. In the following, any operator denoted by $\bar{O}$ will be defined on a lattice, with the convention that $\bar{O} \rightarrow \hat{O}$ in the continuum limit (see Appendix H).

We now apply the pulse sequence in Eq. (36) to the lattice system, by substituting $\hat{p}_{x, y}^{2} / 2 m \rightarrow \bar{p}_{x, y}^{2} / 2 m^{*}$ and $\hat{x} \hat{y} \rightarrow \bar{x} \bar{y}$. The lattice analogue of the sequence (36), which was originally introduced in Ref. [15], now involves pulsed directional hoppings on a lattice, and it can thus be realized using optical-lattice technologies. In this lattice framework, the effective Hamiltonian in Eq. (33) yields

$$
\begin{aligned}
\hat{H}_{\text {eff }}= & -J \sum_{m, n}\left(1-i \pi \Phi n-\frac{4}{3}(\Phi n \pi)^{2}\right) \hat{a}_{m+1, n}^{\dagger} \hat{a}_{m, n}, \\
& +\left(1+i \pi \Phi m-\frac{4}{3}(\Phi m \pi)^{2}\right) \hat{a}_{m, n+1}^{\dagger} \hat{a}_{m, n}+\text { H.c. },
\end{aligned}
$$

where we introduce the "flux" $\Phi=a^{2} \kappa / 8 \omega$ and use the commutators presented in Appendix H 1. In the small flux regime $\Phi \ll 1$, we obtain the Hofstadter Hamiltonian [30]

$$
\begin{aligned}
\hat{H}_{\text {eff }}= & -J \sum_{m, n} e^{-i \pi \Phi n} \hat{a}_{m+1, n}^{\dagger} \hat{a}_{m, n}+e^{i \pi \Phi m} \hat{a}_{m, n+1}^{\dagger} \hat{a}_{m, n}+\text { H.c. } \\
& +\frac{1}{2} m^{*} \omega_{h}^{2}\left(\bar{x}^{2}+\bar{y}^{2}\right)
\end{aligned}
$$

where the additional term acts as a harmonic confinement in the continuum limit, with frequency $\omega_{h}=2 \pi \Phi J \sqrt{5 / 3}$. Noting that $\Phi$ is the number of (synthetic) magnetic flux quanta per unit cell [30], and denoting the cyclotron frequency by $\omega_{c}=B / m^{*}$, we recover the free-space results (38) and (39), namely,

$\boldsymbol{B}=\left(2 \pi \Phi / a^{2}\right) \mathbf{1}_{z}=\frac{\pi \kappa}{4 \omega} \mathbf{1}_{z}, \quad \omega_{h} / \omega_{c}=(1 / 2) \sqrt{5 / 3}$,

which validates the analogy between the free-space and lattice systems. In the present perturbative framework, the flux is limited to $\Phi \ll 1$; however, a partial resummation of the series (42), similar to that of Sec. VII D, allows one to extend the flux range to $\Phi \sim 1$; see Ref. [15].

The lattice system is convenient for physical implementation, since optical lattices offer a platform to activate and deactivate the hopping terms in a controllable manner, e.g., by simply varying the lattice depths in a directional way. Besides, the lattice configuration described in this section also allows for direct numerical simulations of the Schrödinger equation (the lattice discretization being physical). We have performed two types of simulations illustrating (a) the dynamics of a Gaussian wave packet subjected to the pulse sequence (36) and (b) the dynamics of the same wave packet evolving according to the effective Hamiltonian (42) with $\Phi=a^{2} \kappa / 8 \omega$. In Fig. 3, we show the center-of-mass dynamics of a wave packet initially prepared around $\boldsymbol{x}(0)=0$ with a nonzero group velocity 


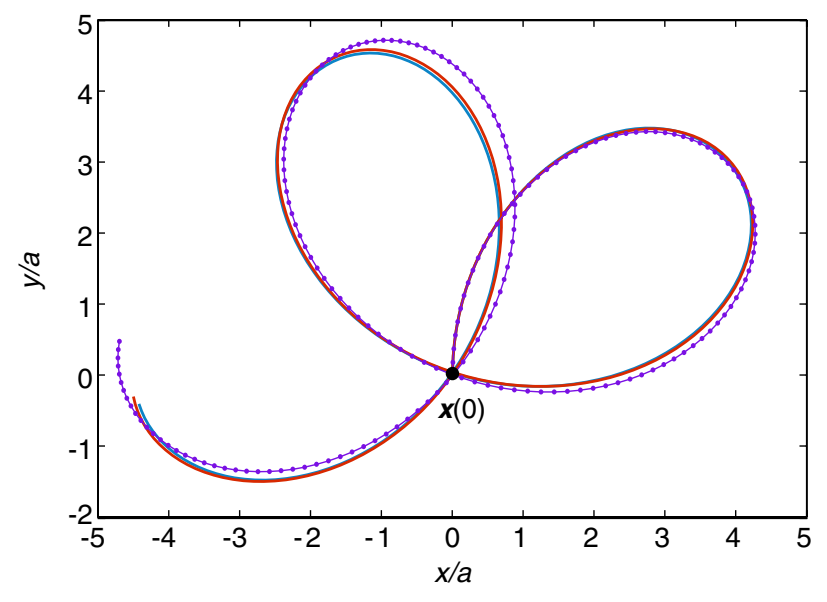

FIG. 3. Comparison between the dynamics of the driven system following the protocol in Eq. (36) (solid red and blue curves) and the dynamics predicted by the effective Hamiltonian (42) (dotted purple curve). Shown is the center-of-mass trajectory in the $x-y$ plane for time $t \in[0,100](1 / J)$. For all simulations, a Gaussian wave packet is initially prepared around $\boldsymbol{x}(0)=0$ with a nonzero group velocity along the $+y$ direction. The blue and red curves correspond to different initial phases of the driving sequence: The blue (respectively, red) curve is obtained by starting the sequence (36) with $\hat{H}_{0}+\hat{A}=\bar{p}_{x}^{2} / m^{*}$ (respectively, $\hat{H}_{0}-\hat{A}=\bar{p}_{y}^{2} / m^{*}$ ). We set the values $\kappa=10$ and $T=\pi / 80(1 / J)$, such as to fulfill the low-flux condition $\Phi=1 / 128 \ll 1$.

$\boldsymbol{v}_{g}(0)=v \mathbf{1}_{y}, v>0$. The nontrivial dynamics associated with the effective Hamiltonian (42) is in very good agreement with the real dynamics of the pulsed system. The spirographic and chiral motion depicted in Fig. 3 is well understood in terms of the effective Hofstadter-like Hamiltonian in Eq. (43): It involves the interplay between the cyclotron motion induced by the synthetic magnetic field $\boldsymbol{B}$ and the presence of the harmonic potential with frequency $\omega_{h}$. Moreover, Fig. 3 compares the trajectories resulting from a different choice of the initial phase of the modulation: The almost identical red and blue trajectories highlight the robustness of this scheme against perturbations in the driving's initial conditions, and incidentally, it shows the negligible role played by the initial kick $\hat{K}\left(t_{i}\right)$ in this example. Similarly, we also note that the micromotion is negligible in real space.

The numerical results presented in Fig. 3 confirm that the driving sequence (36), introduced in Ref. [15], produces an effective magnetic field in the optical-lattice setup; moreover, it illustrates the relevant effects associated with second-order $\left(1 / \omega^{2}\right)$ corrections, which will be present even at low flux $\Phi \ll 1$. Moreover, we point out that adding terms in the static Hamiltonian $\hat{H}_{0}$, for instance, to further control the confinement of the gas or to combine several effects, should be treated with care, as these extra terms will potentially contribute to second-order corrections through the commutators $\left[\left[\hat{A}, \hat{H}_{0}\right], \hat{A}\right]+\left[\left[\hat{B}, \hat{H}_{0}\right], \hat{B}\right]$; see Eq. (33). Finally, we note that the operators $\hat{A}$ and $\hat{B}$ entering the sequence in Eq. (36) could be slightly modified for the sake of experimental simplicity.

\section{GENERATING SYNTHETIC SPIN-ORBIT COUPLINGS IN COLD GASES}

\section{A. Spin-orbit coupling in 2D: General considerations}

In this section, we investigate the possibility to generate spin-orbit-coupling (SOC) terms $\sum_{\mu \nu} \alpha_{\mu \nu} \hat{p}_{\mu} \hat{J}_{\nu}$ in a coldatom gas, where $\hat{\boldsymbol{J}}$ denotes the spin operator associated with the atoms, $\hat{\boldsymbol{p}}$ is the momentum, and $\alpha_{\mu \nu}$ are some coefficients. Here, for the sake of simplicity, the focus will be set on the case of two-dimensional and spin-1/2 Rashba SOC, which is given by $\hat{H}_{R}=\lambda_{R} \hat{\boldsymbol{p}} \cdot \hat{\boldsymbol{\sigma}}$. In the following, we will also encounter another SOC term $\hat{H}_{L \sigma}=\Omega_{\mathrm{SO}} \hat{L}_{z} \hat{\sigma}_{z}$, the so-called "intrinsic" or "helical" SOC, which is responsible for the quantum spin Hall effect in topological insulators [71,72]. The combination of both terms, e.g., as in the Kane-Mele model [72], will be referred to as the "helicalRashba" configuration.

Before presenting different schemes leading to SOC terms, we point out a subtlety associated with Rashba spinorbit-coupled systems that arises when considering a perturbative treatment in powers of $\lambda_{R}$. Consider $\hat{H}_{0}$ the free Hamiltonian in 2D space, and let us perform the following unitary transformation:

$$
\begin{aligned}
e^{-i \hat{G}} \hat{H}_{0} e^{i \hat{G}} & =\hat{H}_{0}+\lambda_{R} \hat{\boldsymbol{p}} \cdot \hat{\boldsymbol{\sigma}}+\Omega_{\mathrm{SO}} \hat{L}_{z} \hat{\sigma}_{z}+m \lambda_{R}^{2}+\mathcal{O}\left(\lambda_{R}^{3}\right), \\
& =\hat{H}_{0}+\hat{H}_{R}+\hat{H}_{L \sigma}+m \lambda_{R}^{2}+\mathcal{O}\left(\lambda_{R}^{3}\right), \\
\hat{H}_{0} & =\hat{p}^{2} / 2 m, \quad \hat{G}=m \lambda_{R} \hat{\boldsymbol{x}} \cdot \hat{\boldsymbol{\sigma}}, \quad \Omega_{\mathrm{SO}}=m \lambda_{R}^{2},
\end{aligned}
$$

where we introduce the short notation $\mathcal{O}\left(\lambda_{R}\right) \equiv \mathcal{O}\left(m \lambda_{R} L\right)$, and $L$ is the system's length. The result in Eq. (45) shows that - up to third order in $\lambda_{R}$ - any model combining the Rashba $\hat{H}_{R}$ and helical $\hat{H}_{L \sigma}$ SOC terms with the weight ratio $\Omega_{\mathrm{SO}} / \lambda_{R}=m \lambda_{R}$ is equivalent to a trivial system described by the free Hamiltonian $\hat{H}_{0}$ [26]. This observation highlights the importance of evaluating the SOC terms up to second order in $\lambda_{R}$, to identify the schemes producing genuinely nontrivial spin-orbit effects. Moreover, in this perturbative framework, Eq. (45) indicates that the (first-order) Rashba SOC Hamiltonian $\hat{H}=\hat{H}_{0}+\hat{H}_{R}$ is equivalent to the (second-order) helical SOC Hamiltonian $\hat{H}=\hat{H}_{0}+\hat{H}_{L \sigma}$, with $\Omega_{\mathrm{SO}}=m \lambda_{R}^{2}$.

Finally, we point out that the expansion in powers of $m \lambda_{R} L$ introduced in Eq. (45) should be handled with care for two main reasons. First, it should not be mistaken with the $(1 / \omega)$ expansion stemming from the effective Hamiltonian formalism of Sec. II A, which is typically characterized by the small dimensionless quantity $\Omega_{\mathrm{SO}} / \omega$. Second, the ground states of the Rashba SOC Hamiltonian $\hat{p}^{2} / 2 m+\lambda_{R} \hat{\boldsymbol{p}} \cdot \hat{\boldsymbol{\sigma}}$ are situated along the "Rashba ring" at $p=p_{R}=m \lambda_{R}$ (i.e., the bottom of the Mexican-hat dispersion $[12,13])$, so that probing this region of the dispersion relation requires us to prepare states with $\Delta p \lesssim m \lambda_{R}$; in this regime, the expansion in Eq. (45) 
becomes problematic as $m \lambda_{R} \Delta x \gtrsim 1 / 2$, which is imposed by Heisenberg inequality.

\section{B. Generating spin-orbit couplings with the $\alpha$ sequence \\ 1. The helical-Rashba scheme}

Inspired by the result presented in Sec. VI A, we propose a scheme to realize spin-orbit couplings, based on the fourstep sequence $\alpha$ in Eq. (32). Considering the operators

$$
\hat{H}_{0}=\hat{p}^{2} / 2 m, \quad \hat{A}=\left(\hat{p}_{x}^{2}-\hat{p}_{y}^{2}\right) / 2 m, \quad \hat{B}=\kappa\left(\hat{x} \hat{\sigma}_{x}-\hat{y} \hat{\sigma}_{y}\right),
$$

the time evolution of the driven system is characterized by the repeated sequence

$$
\left\{\frac{\hat{p}_{x}^{2}}{m}, \hat{H}_{0}+\kappa\left(\hat{x} \hat{\sigma}_{x}-\hat{y} \hat{\sigma}_{y}\right), \frac{\hat{p}_{y}^{2}}{m}, \hat{H}_{0}-\kappa\left(\hat{x} \hat{\sigma}_{x}-\hat{y} \hat{\sigma}_{y}\right)\right\} .
$$

The general expression for the effective Hamiltonian (33) then yields

$$
\begin{aligned}
\hat{H}_{\mathrm{eff}} & =\hat{H}_{0}+\lambda_{R} \hat{\boldsymbol{p}} \cdot \hat{\boldsymbol{\sigma}}+\Omega_{\mathrm{SO}} \hat{L}_{z} \hat{\sigma}_{z}+\mathcal{O}\left(1 / \omega^{3}\right), \\
\lambda_{R} & =\pi \kappa / 8 m \omega, \quad \Omega_{\mathrm{SO}}=-(8 m / 3) \lambda_{R}^{2},
\end{aligned}
$$

providing a helical-Rashba configuration. In this perturbative approach, we note that one can eliminate the Rashba or the helical SOC term in Eq. (48) via a unitary transformation, but not both, since $\Omega_{\mathrm{SO}} \neq m \lambda_{R}^{2}$ (see Sec. VII A). The kick operator $\hat{K}(t)$ is obtained from Eq. (17); in particular, the initial kick at $t_{i}=0$ is given by [Eq. (33)]

$\hat{K}\left(t_{i}=0\right)=-\frac{\pi}{4 \omega}\left[\left(\hat{p}_{x}^{2}-\hat{p}_{y}^{2}\right) / 2 m+\kappa\left(\hat{x} \hat{\sigma}_{x}-\hat{y} \hat{\sigma}_{y}\right)\right]+\mathcal{O}\left(1 / \omega^{2}\right)$.

In direct analogy with the discussion presented in Sec. III, we find that this initial kick can profoundly alter the dynamics of wave packets in the strong SOC regime, where $\kappa / \omega \sim \lambda_{R}$ is large. In this regime, it is thus desirable to launch the dynamics at a subsequent time $t_{i}=3 T / 8$, in which case

$$
\hat{K}\left(t_{i}=3 T / 8\right)=\frac{\pi}{4 \omega}\left[\left(\hat{p}_{x}^{2}-\hat{p}_{y}^{2}\right) / 2 m\right]+\mathcal{O}\left(1 / \omega^{2}\right)
$$

no longer depends on the parameter $\kappa / \omega$.

In the same spirit as in Sec. VIB, we now consider the lattice analogue of the driven system characterized by the pulse sequence (47). The corresponding tight-binding operators and commutators are presented in Appendix $\mathrm{H} 2$. Similarly as in Sec. VIB, this scheme involves a combination of pulsed directional hoppings on the lattice and oscillating quadrupole fields. We obtain that the corresponding effective Hamiltonian $\hat{H}_{\text {eff }}$ reproduces the helicalRashba Hamiltonian in Eq. (48), after substituting $m$ by the effective mass $m^{*}=1 /\left(2 J a^{2}\right)$ and taking the continuum limit. A specificity of the lattice framework is that the second-order contributions also lead to a renormalization of the hopping amplitude $J \rightarrow J\left(1-\eta^{2}\right)$, where $\eta=(a \pi \kappa / 4 \sqrt{3} \omega)$; this specificity is due to the fact that $\left[\left[\bar{p}_{x}^{2}, \bar{x}\right], \bar{x}\right]=\bar{p}_{x}^{2} a^{2}$ in the lattice formulation (Appendix H 2).

We now demonstrate that the dynamics of the pulse sequence (47) is well captured by the predictions of the effective Hamiltonian formalism. We consider that the system is initially prepared in a Gaussian wave packet with nonzero group velocity along the $+x$ direction and spin component $\sigma=+$. Figure 4 compares the dynamics generated by the pulse sequence (47) with the one associated with the evolution operator in Eq. (15), with $\hat{H}_{\text {eff }}$ and $\hat{K}(t)$ given by Eqs. (17), (48), and (G4). We show in Figs. 4(a) and 4(b) the spin populations as a function of time: Using a time step $\Delta t<T$, we observe a wide micromotion in spin space, which is very well captured by the kick operator $\hat{K}(t)$ of the effective model. Figure 4(c) compares the center-of-mass motion of the real and effective evolution operators. The curved trajectory, together with the evolution of the spin populations, signals the presence of the effective SOC generated by the driving. In agreement with the discussion of Sec. VII A, we recover the fact that it is necessary to evaluate the effective Hamiltonian up to (at least) second order in $1 / \omega$ to reach a good agreement with the dynamics of the real pulsed system. Adding third-order corrections to the effective Hamiltonian [Eq. (G4) in Appendix G] leads to an even better agreement. Finally, similarly as in Fig. 3, we find that the micromotion is small in real space.

\section{The pure Dirac regime}

Interestingly, Eq. (48) suggests that inhibiting the effect of $\hat{H}_{0}$ during the evolution will lead to a pure Dirac system

$$
\hat{H}_{\mathrm{eff}}^{D}=\lambda_{R} \hat{\boldsymbol{p}} \cdot \hat{\boldsymbol{\sigma}}+\mathcal{O}\left(1 / \omega^{3}\right),
$$

noting that the helical SOC term in Eq. (48) is due to the commutator $\left[\left[\hat{B}, \hat{H}_{0}\right], \hat{B}\right]$ in Eq. (33). This Dirac Hamiltonian is obtained with the pulse sequence

$$
\begin{aligned}
& \left\{\left(\bar{p}_{x}^{2}-\bar{p}_{y}^{2}\right) / 2 m, \kappa\left(\bar{x} \hat{\sigma}_{x}-\bar{y} \hat{\sigma}_{y}\right),\right. \\
& \left.\quad-\left(\bar{p}_{y}^{2}-\bar{p}_{x}^{2}\right) / 2 m,-\kappa\left(\bar{x} \hat{\sigma}_{x}-\bar{y} \hat{\sigma}_{y}\right)\right\},
\end{aligned}
$$

which, in principle, can be realized in an optical-lattice setup. Hence, controlling the static Hamiltonian $\hat{H}_{0}$, which is conceivable in a lattice framework, offers the possibility to tune the first-order effective energy spectrum but also to annihilate the second-order contributions to the SOC effects. We note that the pulsed operators $\hat{A}$ and $\hat{B}$ in Eq. (46) could also be slightly modified to reach other regimes of interest. 

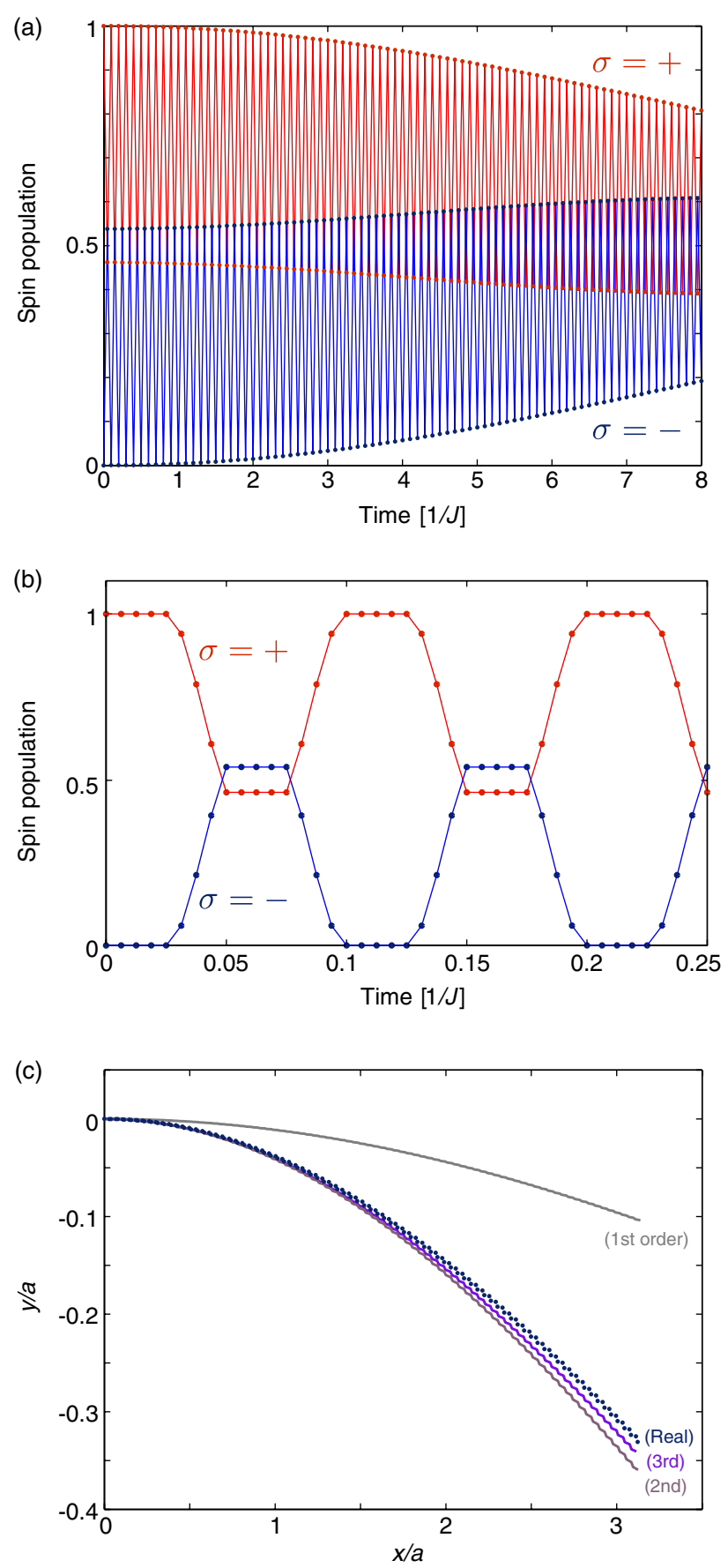

FIG. 4. The helical-Rashba scheme: dynamics of the driven system associated with the driving sequence (47). Here, $\kappa=10 \mathrm{~J} / a$ and $T=$ $0.1 / J$. (a) Spin populations as a function of time. The dynamics of the pulsed system (blue and red dots) is compared with the evolution dictated by the evolution operator in Eq. (15) (blue and red lines), where $\hat{H}_{\text {eff }}$ and $\hat{K}(t)$ are given by Eqs. (17), (48), and (G4). The effective and real dynamics are sampled using the time step $\Delta t=T / 2$. (b) Same as above, but using a time step $\Delta t=T / 16$ to highlight the micromotion captured by the kick operator $\hat{K}(t)$ in Eq. (17). (c) Center-of-mass displacement in the $x$ - $y$ plane after a time $t=8 / J$. Here, the real trajectory of the driven system (blue dots) is compared with the evolution predicted by the effective Hamiltonian formalism [Eq. (15)], considering $\hat{H}_{\text {eff }}$ at first, second, and third order in $1 / \omega$. The trajectories are sampled using the time step $\Delta t=T / 2$.
3. Adding terms to the static Hamiltonian $\hat{H}_{0}:$ A route toward topological superfluids and topological insulators

The scheme based on the driving sequence (46) and (47) allows for adding a Zeeman term $\hat{H}_{Z}=\lambda_{Z} \hat{\sigma}_{z}$ to the effective Hamiltonian (48), whose association with the Rashba SOC term could be useful for the quantum simulation of topological superfluids [73]. Subjecting the system to a static Zeeman field $\hat{H}_{0} \rightarrow \hat{H}_{0}+\hat{H}_{Z}$ will not perturb the first-order Rashba term $\hat{H}_{R} \sim[\hat{A}, \hat{B}]$ in Eq. (48) but will add an extra second-order term

$$
\begin{aligned}
\hat{H}_{\text {eff }}= & \hat{H}_{0}+\hat{H}_{Z}+\hat{H}_{R}+\hat{H}_{L \sigma} \\
& +\frac{1}{2} m \Omega_{Z}^{2}\left(x^{2}+y^{2}\right) \hat{\sigma}_{z}+\mathcal{O}\left(1 / \omega^{3}\right),
\end{aligned}
$$

which corresponds to a spin-dependent harmonic potential with frequency $\Omega_{Z}=2 \sqrt{\lambda_{Z} \Omega_{\text {SO }}}$. The survival of the topological superfluid phase in the presence of the additional harmonic potential constitutes an interesting open question. In general, we note that any driving scheme aiming to produce Rashba SOC typically presents this potential drawback, namely, the fact that the additional Zeeman term will necessarily generate additional (possibly spoiling) effects.

Adding terms to the static Hamiltonian $\hat{H}_{0}$ could also be envisaged to generate systems exhibiting the anomalous quantum Hall effect $[38,74]$, the so-called Chern insulators. For instance, the anomalous quantum Hall model of Ref. [74] could be realized by considering the driving sequence (46) and (47) on a square lattice but replacing the static Hamiltonian $\hat{H}_{0} \rightarrow \hat{H}_{Z}+\left(\bar{p}^{2} / 2 m\right) \hat{\sigma}_{z}$, where the last term corresponds to a spin-dependent hopping term on the lattice. Another possibility would be to drive lattice systems with more complex geometries (e.g., honeycomb lattice), where the association of Rashba and Zeeman terms directly leads to Chern insulating phases $[75,76]$.

\section{The xy scheme}

Finally, we introduce a second scheme based on the $\alpha$ sequence (32), which features the standard static Hamiltonian $\hat{H}_{0}=\hat{p}^{2} / 2 m$ and the pulsed operators

$$
\begin{aligned}
& \hat{A}=\left(\hat{p}_{y}^{2}-\hat{p}_{x}^{2}\right) / 2 m-\kappa \hat{x} \hat{\sigma}_{x}, \\
& \hat{B}=\left(\hat{p}_{y}^{2}-\hat{p}_{x}^{2}\right) / 2 m-\kappa \hat{y} \hat{\sigma}_{y} .
\end{aligned}
$$

The repeated driving sequence is similar to Eq. (47), reading

$$
\left\{\frac{\hat{p}_{y}^{2}}{m}-\kappa \hat{x} \hat{\sigma}_{x}, \frac{\hat{p}_{y}^{2}}{m}-\kappa \hat{y} \hat{\sigma}_{y}, \frac{\hat{p}_{x}^{2}}{m}+\kappa \hat{x} \hat{\sigma}_{x}, \frac{\hat{p}_{x}^{2}}{m}+\kappa \hat{y} \hat{\sigma}_{y}\right\},
$$

so that this scheme is based on a more regular sequence involving pulsed directional motion and magnetic fields. The effective Hamiltonian (33) then reads 


$$
\begin{aligned}
\hat{H}_{\mathrm{eff}} & =\hat{H}_{0}+\lambda_{R} \hat{\boldsymbol{p}} \cdot \hat{\boldsymbol{\sigma}}-\gamma \hat{x} \hat{y} \hat{\sigma}_{z}+\mathrm{cst}+\mathcal{O}\left(1 / \omega^{3}\right), \\
\lambda_{R} & =-\pi \kappa / 8 m \omega, \quad \gamma=\pi \kappa^{2} / 4 \omega .
\end{aligned}
$$

Here, the second-order terms do not contribute to the effective Hamiltonian, but the first-order terms include a spin-dependent hyperbolic potential. Although the " $x y$ " term is potentially problematic, especially in the strong SOC regime $\kappa / \omega \gg$, we will show in Sec. VII D that this pulse sequence allows for an almost exact treatment; incidentally, we will see that the $x y$ term can be inhibited in the lattice framework through a fine-tuning of the driving parameters.

\section{Generating spin-orbit couplings with the $\beta$ sequence: The XA scheme}

The XA scheme introduced by Xu et al. in Ref. [26] and Anderson et al. in Ref. [25] is based on a repeated pulse sequence, which can be expressed through the evolution operator over one period $T$

$$
\begin{aligned}
\hat{U}(T)= & e^{-i\left(\hat{H}_{0}-\hat{B}\right) \tau} e^{-i \hat{H}_{0} \tilde{T} / 2} e^{-i\left(\hat{H}_{0}+\hat{B}\right) \tau} \\
& \times e^{-i\left(\hat{H}_{0}-\hat{A}\right) \tau} e^{-i \hat{H}_{0} \tilde{T} / 2} e^{-i\left(\hat{H}_{0}+\hat{A}\right) \tau},
\end{aligned}
$$

where $\tau \ll T \approx \tilde{T}$, and where the operators are explicitly given by

$$
\hat{H}_{0}=\hat{p}^{2} / 2 m, \quad \hat{A}=\kappa \hat{x} \hat{\sigma}_{x}, \quad \hat{B}=\kappa \hat{y} \hat{\sigma}_{y} .
$$

Noting that the sequence (53) essentially features four nontrivial steps (with pulses $\pm \hat{A}$ and $\pm \hat{B}$ ), we find that it can be qualitatively described by the associated four-step sequence $\beta$ in Eq. (34). The driving sequence studied in this section, hereafter referred to as the XA scheme, will thus be taken of the form $\beta$ :

$$
\left\{\hat{H}_{0}+\kappa \hat{x} \hat{\sigma}_{x}, \hat{H}_{0}-\kappa \hat{x} \hat{\sigma}_{x}, \hat{H}_{0}+\kappa \hat{y} \hat{\sigma}_{y}, \hat{H}_{0}-\kappa \hat{y} \hat{\sigma}_{y}\right\} .
$$

The corresponding effective Hamiltonian and initial-kick operators then read [Eq. (35)]

$$
\begin{aligned}
\hat{H}_{\mathrm{eff}} & =\hat{H}_{0}-\Omega_{\mathrm{SO}} \hat{L}_{z} \hat{\sigma}_{z}+\mathcal{O}\left(1 / \omega^{3}\right), \quad \Omega_{\mathrm{SO}}=m \lambda_{R}^{2}, \\
\hat{K}(0) & =-m \lambda_{R} \hat{\boldsymbol{x}} \cdot \hat{\boldsymbol{\sigma}}+\mathcal{O}\left(1 / \omega^{2}\right), \quad \lambda_{R}=\pi \kappa / 8 m \omega,
\end{aligned}
$$

where the absence of first-order (Rashba) terms is a characteristic of the $\beta$ sequence [Eq. (35)]. However, using Eq. (15) and comparing Eqs. (56) and (45), one recovers an effective Rashba SOC term via the unitary transform $[\hat{K}(T)=\hat{K}(0)]$

$$
\begin{aligned}
\hat{U}(T) & =e^{-i \hat{K}(T)} e^{-i T \hat{H}_{\mathrm{eff}}} e^{i \hat{K}(0)}=e^{-i T \hat{H}_{\mathrm{eff}}^{T}}, \\
\hat{H}_{\mathrm{eff}}^{\mathcal{T}} & =\hat{H}_{0}-\lambda_{R} \hat{\boldsymbol{p}} \cdot \hat{\boldsymbol{\sigma}}+\mathcal{O}\left(1 / \omega^{3}\right)+\mathcal{O}\left(\lambda_{R}^{3}\right),
\end{aligned}
$$

where we have introduced the effective Hamiltonian $\hat{H}_{\text {eff }}^{\mathcal{T}}$. As anticipated at the end of Sec. VII A, Eq. (57) implicitly contains two intertwined perturbative expansions: the expansion in powers of $\left(\Omega_{\mathrm{SO}} / \omega\right)$ inherent to $\hat{H}_{\text {eff }}$ and $\hat{K}(t)$ (Sec. II A) and the expansion in powers of $\left(m \lambda_{R} L\right)$ introduced in Eq. (45).

\section{Quasiexact treatments on special cases}

In this section, we show that specific driving schemes leading to SOC benefit from the fact that they can be treated almost exactly. Such an approach is useful, as it allows us to go beyond the perturbative treatment of Sec. II A, which has been considered until now to evaluate the evolution operator. Actually, we have already encountered such a scheme in our study of the oscillating force in Sec. III.

\section{The XA scheme}

Following Refs. [25,26], the time-evolution operator associated with the sequence (55) can be conveniently partitioned as $\hat{U}(T)=\hat{U}_{y} \hat{U}_{x}$, where each of the two subsequences

$\hat{U}_{\mu}=e^{-i\left(\hat{H}_{0}-\kappa \hat{\mu} \hat{\sigma}_{\mu}\right) T / 4} e^{-i\left(\hat{H}_{0}+\kappa \hat{\mu} \hat{\sigma}_{\mu}\right) T / 4}, \quad \mu=x, y$,

can be treated exactly. The calculations presented in Appendix I yield the exact result (see also Refs. [25,26])

$$
\hat{U}_{\mu}=e^{-i T\left[\hat{H}_{0} / 2-\lambda_{R} \hat{p}_{\mu} \hat{\sigma}_{\mu}\right]}, \quad \mu=x, y,
$$

where $\lambda_{R}=\pi \kappa / 8 m \omega$. Finally, the evolution operator after one period is obtained by using the Trotter expansion to the lowest order $\exp A \exp B \approx \exp (A+B)$, reading

$$
\begin{aligned}
\hat{U}(T) & =\hat{U}_{y} \hat{U}_{x}=\exp \left(-i \hat{H}_{\mathrm{eff}}^{\mathcal{T}} T\right), \\
\hat{H}_{\mathrm{eff}}^{\mathcal{T}} & =\hat{H}_{0}-\lambda_{R} \hat{\boldsymbol{p}} \cdot \hat{\boldsymbol{\sigma}}+\mathcal{O}\left[\left(\Omega_{\mathrm{SO}} / \omega\right)^{2}\right] .
\end{aligned}
$$

We thus recover the result in Eq. (57), with the notable difference that the quasiexact treatment leads to a partial resummation of the infinite series inherent to Eq. (57): Indeed, the dimensionless parameter $m \lambda_{R} L$ no longer plays any role in the expression for the evolution operator in Eq. (60).

Before discussing the result in Eq. (60) any further, we derive its lattice analogue by substituting the operators of the XA sequence (55) by their lattice counterparts (Appendix H2). Following the computations presented in Appendix I, we obtain the effective Hamiltonian up to first order in $\left(\Omega_{\mathrm{SO}} / \omega\right)^{2}$

$$
\begin{aligned}
\hat{U}(T)= & \bar{U}_{y} \bar{U}_{x}=\exp \left[-i \bar{H}_{\mathrm{eff}}^{\mathcal{T}} T\right], \\
\bar{H}_{\mathrm{eff}}^{\mathcal{T}}= & \frac{\bar{p}^{2}}{2 m^{*}}\left\{\frac{1}{2}+\frac{1}{2} \operatorname{sinc}\left(4 a m^{*} \lambda_{R}\right)\right\} \\
& +\overline{\boldsymbol{p}} \cdot \hat{\boldsymbol{\sigma}}\left\{\frac{\cos \left(4 a m^{*} \lambda_{R}\right)-1}{8\left(a m^{*}\right)^{2} \lambda_{R}}\right\},
\end{aligned}
$$

where $\lambda_{R}=\pi \kappa / 8 m^{*} \omega$ and $m^{*}=1 / 2 J a^{2}$ is the effective mass. Hence, we recover the result in Eq. (60) for weak 
driving $\lambda_{R}<a J / 2$ and by taking the continuum limit. However, in the lattice framework, the maximum value of the effective Rashba SOC strength is limited: Using Eq. (61), we find that the ratio of Rashba to hopping is maximized for $\lambda_{R}=\pi / 4 a m^{*}=(\pi / 2) a J$ :

$$
\bar{H}_{\mathrm{eff}}^{\mathcal{T}}=\frac{1}{2}\left(\frac{\bar{p}^{2}}{2 m^{*}}\right)-\lambda_{R}^{*} \overline{\boldsymbol{p}} \cdot \hat{\boldsymbol{\sigma}}, \quad \lambda_{R}^{*}=(2 / \pi) a J
$$

Note that the appearance of sinc functions in Eq. (61) is a characteristic of lattice systems driven by square-wave modulations [67].

The quasiexact method presented in this section allows us to partially resum the infinite series contained in $\hat{H}_{\text {eff }}$ and $\hat{K}(t)$ [Eq. (57)]. However, we stress that the evolution operators and the associated effective Hamiltonians in Eqs. (60) and (61) depend on the initial phase of the modulation, in direct analogy with the situation presented in Secs. I B and III: The analysis performed in this section imposes that the $\beta$ sequence exactly starts with the pulse $+\hat{A}$ and ends with the pulse $-\hat{B}$. Any deviation in the initial phase will alter the evolution operator in Eqs. (60) and (61), and potentially, the long-time dynamics. Indeed, suppose that the launching time is shifted $t_{i}=0 \rightarrow-T / 4$, so that the $\beta$ sequence starts with the pulse $-\hat{B}$ instead of $+\hat{A}$ : The system will first undergo a kick

$$
\exp \left(i \kappa T \hat{y} \hat{\sigma}_{y} / 4\right)=\exp \left(i \delta p \hat{y} \hat{\sigma}_{y}\right), \quad \delta p=4 m \lambda_{R},
$$

before evolving according to the Rashba Hamiltonian in Eqs. (60) and (61). Note that $\delta p=4 p_{R}$, where $p_{R}$ is the radius of the Rashba ring along which the ground states are situated. Hence, this initial kick, which modifies the group velocity and spin structure of the prepared system, typically has an impact on long-time dynamics (in direct analogy with Fig. 1). We illustrate this sensitivity to the initial phase of the driving in Fig. 5, which shows the time-evolved

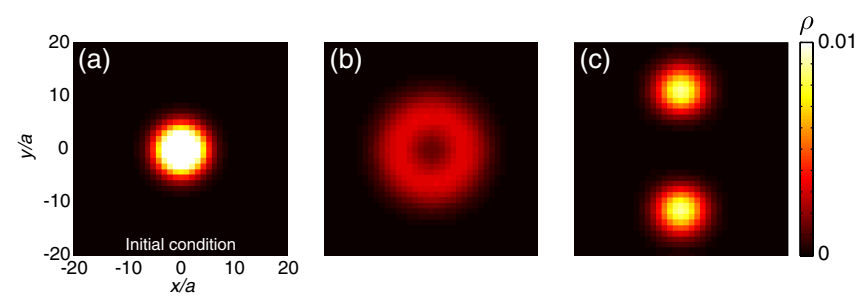

FIG. 5. The dynamics associated with the driving sequence (55) on a lattice. (a) The Gaussian wave packet is initially prepared around $\boldsymbol{x}(0)=0$ and momentum $\boldsymbol{k}=0$, so as to probe the Dirac dispersion relation of the effective Rashba Hamiltonian in Eq. (61) $\left(\Delta k \ll m^{*} \lambda_{R}\right)$. (b), (c) Shown is the time-evolved particle density $\rho(x, y)$ in the $x-y$ plane at time $t=7.3(1 / J)$, for $\kappa=40 J / a$, and $T=7 \pi / 100(1 / J)$, i.e., $\lambda_{R} \approx(\pi / 3) J a$. (b) The driving follows the sequence (55) starting with the pulse $+\hat{A}$. (c) Same but starting with the pulse $-\hat{B}$, i.e., changing the initial phase of the modulation. density of a lattice system driven by the sequence (55). Figure 5(a) shows the initial wave packet in real space, with mean position $\boldsymbol{x}=0$ and momentum $\boldsymbol{k}=0$. The width of the Gaussian satisfies $\Delta k \ll m^{*} \lambda_{R}$ in $k$ space; namely, the wave packet is prepared such as to probe the Dirac dispersion relation around $\boldsymbol{k}=0$ (i.e., within the Rashba ring). Figure 5(b) shows the time-evolved density at time $t=7.3 / J$, in the case where the $\beta$ sequence (55) is launched with the pulse $+\hat{A}$ : In agreement with the evolution operator in Eq. (61), the cloud expands according to the isotropic Dirac dispersion relation associated with the effective Rashba Hamiltonian $\bar{H}_{\text {eff }}^{\mathcal{T}}$. Figure 5(c) shows the same time-evolution protocol but for a driving sequence $\beta$ launched with the pulse $-\hat{B}$ (i.e., shifting the launching time $t_{i}=0 \rightarrow-T / 4$ ). As predicted by Eq. (63), the cloud undergoes a sudden kick along the $y$ direction, which ejects the initial momentum distribution out of the Rashba ring, before evolving according to the Rashba Hamiltonian (61); hence, changing the initial phase of the modulation results in a highly anisotropic dynamics that no longer probes the Dirac dispersion relation proper to the Rashba Hamiltonian (61).

We also note that the treatment considered in this section implies that the system is probed stroboscopically $(t=N T)$, and in this sense, it does not describe the micromotion associated with the driving. More importantly, we point out that the exact treatment leading to Eq. (59) cannot be performed when adding Pauli matrices into the static Hamiltonian (e.g., an extra Zeeman term). Finally, we point out that a similar quasiexact treatment was considered in Ref. [15], for the one-component driven lattice discussed in Sec. VI.

\section{The xy scheme}

The $x y$ scheme introduced in Sec. VII B 4 can also be treated in an almost exact manner, which leads to a partial resummation of the series in Eq. (52). The method differs from the one presented in Sec. VII D 1 for the XA scheme, and importantly, it is only possible under two conditions: (1) The $x y$ sequence in Eq. (51) should be realized on the lattice, in which case all the operators are replaced by their lattice analogue (Appendix H2). (2) The driving parameter $\kappa$ and the period $T$ should satisfy the condition $\kappa T=4 \pi / a$, or equally, using our previous definition, $\lambda_{R}=\pi \kappa / 8 m^{*} \omega=(\pi / 2) a J$. We note that this particular value was already discussed below Eq. (61), where it corresponds to the regime where the effective Rashba SOC is maximized on the lattice. In the following, we assume that these two conditions are satisfied.

Here, in contrast with the analysis performed in Sec. VII D 1, we split the evolution operator $\bar{U}(T)$ associated with the sequence (51) into its four primitive parts

$$
\bar{U}(T)=\bar{U}_{-B} \bar{U}_{-A} \bar{U}_{+B} \bar{U}_{+A},
$$


and we analyze each operator $\bar{U}_{ \pm A, B}$ separately. Using the Zassenhaus formula [77], we find factorized expressions for the individual operators (see Appendix J for details)

$$
\begin{array}{ll}
\bar{U}_{+A}=e^{-i T \bar{p}_{y}^{2} / 4 m^{*}} e^{i \pi \bar{x} / a}, & \bar{U}_{-A}=e^{i \pi \bar{x} / a} e^{i T \lambda_{R}^{*} \bar{p}_{x} \bar{\sigma}_{x},} \\
\bar{U}_{+B}=e^{i \pi \bar{y} / a} e^{-i T \lambda_{R}^{*} \bar{p}_{y} \bar{\sigma}_{y}}, & \bar{U}_{-B}=e^{-i T \bar{p}_{x}^{2} / 4 m^{*}} e^{i \pi \bar{y} / a},
\end{array}
$$

where $\lambda_{R}^{*}$ was defined in Eq. (62). Finally, using Eq. (H6), and applying the Trotter expansion to minimal order, we find

$$
\begin{aligned}
\bar{U}(T) & =\bar{U}_{-B} \bar{U}_{-A} \bar{U}_{+B} \bar{U}_{+A}=\exp \left(-i \bar{H}_{\mathrm{eff}}^{\mathcal{T}} T\right), \\
\bar{H}_{\mathrm{eff}}^{\mathcal{T}} & =\frac{1}{2}\left(\frac{\bar{p}^{2}}{2 m^{*}}\right)+\lambda_{R}^{*} \overline{\boldsymbol{p}} \cdot \hat{\boldsymbol{\sigma}}+\mathcal{O}\left[\left(\Omega_{\mathrm{SO}} / \omega\right)^{2}\right],
\end{aligned}
$$

which is precisely the Rashba Hamiltonian in Eq. (62) up to the sign change $\lambda_{R}^{*} \rightarrow-\lambda_{R}^{*}$ (i.e., a gauge transformation). Interestingly, this result shows that the $x y$ scheme in Eq. (51) is equivalent to the XA scheme in Eq. (55), in the limit $\lambda_{R} \rightarrow(\pi / 2) a J$, where the Rashba SOC is maximized. Furthermore, Eq. (66) shows that the hyperbolic potential, which is predicted by the perturbative treatment [Eq. (52)] for the lattice-free case, totally disappears in this special regime;
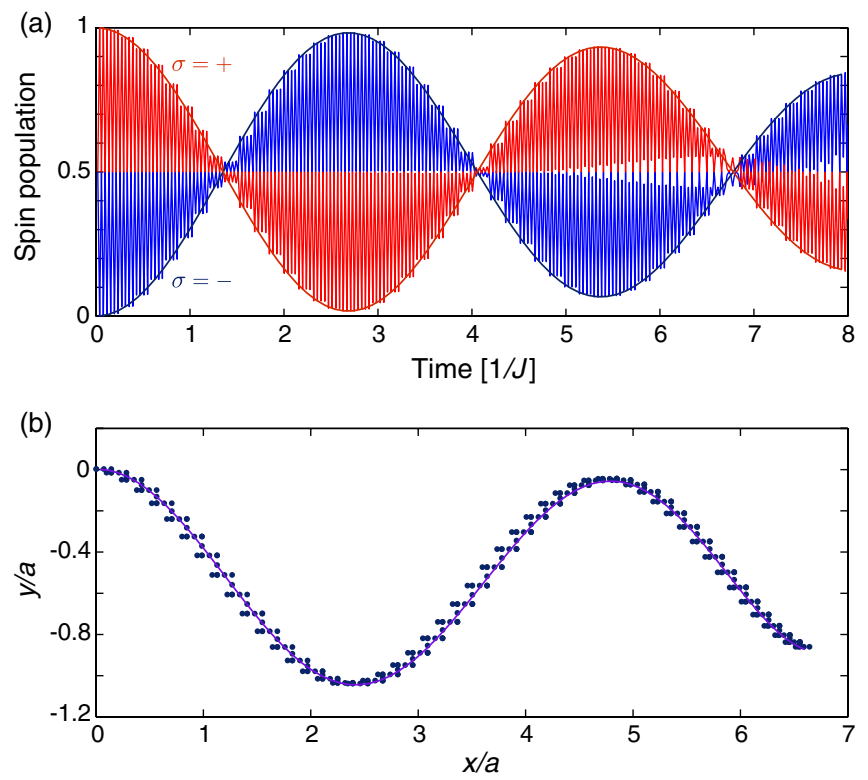

FIG. 6. The $x y$ scheme: dynamics of the driven system associated with the driving sequence (51). Here, $\kappa=80 \mathrm{~J} / \mathrm{a}$ and $T=\pi / 20 J^{-1}$, which corresponds to the special regime where $\lambda_{R}=(\pi / 2) a J$. (a) Spin populations as a function of time. Bold (respectively, thin) lines correspond to the predictions of the effective Hamiltonian in Eq. (66) (respectively, to the real dynamics of the pulsed system). (b) Center-of-mass displacement in the $x-y$ plane after a time $t=8 / J$. The blue dots (respectively, purple line) correspond to the real dynamics of the pulsed system [respectively, to the effective Hamiltonian in Eq. (66)]. In both figures, the real dynamics are sampled using the time step $\Delta t=$ $T / 8$ to highlight the micromotion, which is not captured by the effective Hamiltonian. this surprising result is due to the underlying lattice structure (Appendix J).

We show in Fig. 6 the perfect agreement between the dynamics predicted by the effective Hamiltonian (66) and the real dynamics of the pulsed-lattice system. One should note that this agreement is only valid in the special regime where $\lambda_{R} \approx(\pi / 2) a J$ : The spoiling effects associated with the effective hyperbolic potential [Eq. (52)] become appreciable as soon as $\Delta \lambda_{R} \sim 1 \%$. Finally, we stress that the analysis leading to Eq. (66) cannot be performed when the static Hamiltonian $\hat{H}_{0}$ features Pauli matrices (e.g., a Zeeman term).

\section{DISCUSSIONS AND CONCLUSIONS}

\section{A. Convergence of the $(1 / \omega)$ expansion}

As already pointed out in Ref. [59], one cannot affirm that the perturbative expansion proper to the formalism of Sec. II A converges, in general. Indeed, the small dimensionless parameter associated with the $1 / \omega$ expansion that leads to the effective Hamiltonian $\hat{H}_{\text {eff }}$ in Eq. (16) can only be determined a posteriori, on a case-by-case basis.

\section{Illustration of the convergence issue}

Let us illustrate this fact based on the driven system discussed in Sec. VI A. Using Eq. (16), we obtain the second-order effective Hamiltonian in Eq. (37), which we now decompose in terms of the $1 / \omega$ expansion:

$$
\begin{aligned}
\hat{H}_{\mathrm{eff}} & =\hat{H}^{0}+\hat{H}^{1}+\hat{H}^{2}+\mathcal{O}\left(1 / \omega^{3}\right), \\
\hat{H}^{0} & =\frac{\hat{p}_{x}^{2}}{2 m}, \quad \hat{H}^{1}=-\Omega\left(\hat{x} \hat{p}_{y}-\hat{y} \hat{p}_{x}\right), \\
\hat{H}^{2} & =\frac{1}{2} m \omega_{\mathrm{conf}}^{2}\left(\hat{x}^{2}+\hat{y}^{2}\right),
\end{aligned}
$$

where $\Omega \sim \kappa / m \omega$ and $\omega_{\text {conf }}^{2} \sim \Omega^{2}$. At this point, no element justifies the fact that the perturbative expansion should be carried up to second order. In order to simplify the present discussion, we slightly modify the sequence in Eq. (36) so that the Hamiltonian in Eq. (67) can be recast in the familiar form

$$
\begin{aligned}
\hat{H}_{\text {eff }} & =(\hat{\boldsymbol{p}}-\mathcal{A})^{2} / 2 m+\mathcal{O}\left(1 / \omega^{3}\right), \\
\mathcal{A} & =\Omega m(-\hat{y}, \hat{x}, 0),
\end{aligned}
$$

which is easily done by changing the prefactor in front of the pulse operator $\hat{A} \sim \hat{p}_{x}^{2}-\hat{p}_{y}^{2}$ entering the sequence in Eq. (36). Having obtained the effective Hamiltonian in Eq. (68) up to second order in $1 / \omega$, one can determine its general characteristics: For instance, let us focus on its ground state, which is the standard lowest Landau level (LLL) with cyclotron frequency $\Omega$. This ground state is characterized by the cyclotron radius $r_{0} \sim 1 / \sqrt{m \Omega}$, such that the typical momentum associated with this state is 
$p_{0} \sim \sqrt{m \Omega} \sim \sqrt{\kappa / \omega}$. Hence, as far as the LLL is concerned, we find that all the terms in Eq. (67) are of the same order $\hat{H}^{0,1,2} \sim \Omega \sim \kappa / m \omega$, which indicates that the perturbative expansion leading to Eq. (67) should necessarily be undertaken up to second order (included). Building on this result, we now evaluate the third-order terms, which have been neglected up to now: Including the pulse operators $\hat{A} \sim \hat{p}_{x}^{2}-\hat{p}_{y}^{2}$ and $\hat{B} \sim \hat{x} \hat{y}$ into Eqs. (G3) and (G4), we find a cancellation of all the third-order terms. We thus need to evaluate the fourth-order terms, which are necessarily of the form

$$
\begin{aligned}
& \frac{1}{\omega^{4}}[[[[\hat{\mathcal{Q}}, \hat{B}], \hat{B}], \hat{\mathcal{Q}}], \hat{\mathcal{Q}}] \sim \frac{\kappa^{2} p^{2}}{\omega^{4} m^{3}}, \\
& \frac{1}{\omega^{4}}[[[[\hat{\mathcal{Q}}, \hat{B}], \hat{B}], \hat{\mathcal{Q}}], \hat{B}] \sim \frac{\kappa^{3} x^{2}}{\omega^{4} m^{2}}, \\
& \frac{1}{\omega^{4}}[[[[\hat{\mathcal{Q}}, \hat{B}], \hat{\mathcal{Q}}], \hat{B}], \hat{\mathcal{Q}}] \sim \frac{\kappa^{2} p^{2}}{\omega^{4} m^{3}}, \\
& \frac{1}{\omega^{4}}[[[[\hat{\mathcal{Q}}, \hat{B}], \hat{\mathcal{Q}}], \hat{B}], \hat{B}] \sim \frac{\kappa^{3} x^{2}}{\omega^{4} m^{2}},
\end{aligned}
$$

where $\hat{\mathcal{Q}}=\left(\hat{H}_{0}, \hat{A}\right) \sim p^{2} / m$. In the LLL, these fourthorder terms are all of order $\Omega^{2} / \omega$ or $\Omega^{3} / \omega^{2}$. Hence, we have identified that $\Omega / \omega \sim \kappa / m \omega^{2}$ constitutes the relevant dimensionless parameter in the problem: The perturbative expansion leading to the effective Hamiltonian in Eq. (67) can indeed be safely limited to the second order as long as $\Omega \ll \omega$, namely, when $\kappa \ll m \omega^{2}$.

Based on this example, we propose a guideline that should be followed in order to validate the convergence of the perturbative expansion. 1. Compute the effective Hamiltonian $\hat{H}_{\text {eff }}$ up to some order $1 / \omega^{n}$, using the formalism presented in Sec. II A. 2. Determine the interesting characteristics associated with $\hat{H}_{\text {eff }}$, e.g., based on its eigenstates or its dispersion relation. 3. Evaluate the $(n+1)$-order term and identify the condition according to which this term can (possibly) be neglected. This condition defines the small dimensionless parameter of the problem, or equally, a region in the parameter space, out of which higher-order terms could come into play.

\section{The perturbative approach revisited}

We emphasize an important aspect related to timedependent Hamiltonians $\hat{H}(t)=\hat{H}_{0}+\sum_{m} f_{m}(\omega t) \hat{V}_{m}$, which is the fact that the operators $\hat{H}_{0}$ and $\hat{V}_{m}$ are generally not independent with respect to the modulation frequency $\omega$. For instance, in the example discussed above in Sec. VIII A 1 , we find that $\hat{H}_{0} \sim \hat{A} \sim \Omega$ and $\hat{B}=\kappa \hat{x} \hat{y} \sim \omega$, based on the LLL characteristics (with $\Omega \ll \omega$ ). The fact that some driving operators may be proportional to the frequency $\hat{V}_{m} \sim \omega$, which can only be determined a posteriori according to the guideline established above, seems problematic when building a perturbative expansion in powers of $1 / \omega$ (Sec. II A). To treat this seemingly pathological situation, we propose an alternative perturbative approach in Appendix $\mathrm{K}$ that is specifically dedicated to the general time-dependent problem

$$
\hat{H}(t)=\hat{H}_{0}+\hat{\mathcal{A}} f(t)+\omega \hat{\mathcal{B}} g(t)
$$

where the functions $f$ and $g$ are assumed to be time periodic with a zero mean value over one period $T=2 \pi / \omega$. Applying this alternative method to lowest order in $1 / \omega$ provides a compact form for the effective Hamiltonian $\hat{H}_{\text {eff }}=\hat{H}_{\text {eff }}^{0}+\mathcal{O}(1 / \omega)$, where

$$
\begin{aligned}
\hat{H}_{\mathrm{eff}}^{0}= & \overline{\exp (i G(t) \hat{\mathcal{B}})\left\{\hat{H}_{0}+\hat{\mathcal{A}} f(t)\right\} \exp (-i G(t) \hat{\mathcal{B}})} \\
= & \hat{H}_{0}+i \overline{G f}[\hat{\mathcal{B}}, \hat{\mathcal{A}}]-\frac{1}{2} \overline{G^{2}}\left[\hat{\mathcal{B}},\left[\hat{\mathcal{B}}, \hat{H}_{0}\right]\right] \\
& -\frac{1}{2} \overline{G^{2} f}[\hat{\mathcal{B}},[\hat{\mathcal{B}}, \hat{\mathcal{A}}]]-\frac{i}{6} \overline{G^{3}}\left[\hat{\mathcal{B}}\left[\hat{\mathcal{B}},\left[\hat{\mathcal{B}}, \hat{H}_{0}\right]\right]\right] \\
& -\frac{i}{6} \overline{G^{3} f}[\hat{\mathcal{B}}[\hat{\mathcal{B}},[\hat{\mathcal{B}}, \hat{\mathcal{A}}]]+\cdots, \\
\hat{K}(t)= & G(t) \hat{\mathcal{B}}+\mathcal{O}(1 / \omega),
\end{aligned}
$$

where $\overline{\zeta(t)}=(1 / T) \int_{0}^{T} \zeta(t) d t$ denotes the mean value over one period, and where $G(t)=\omega \int{ }^{t} g(\tau) d \tau$ satisfies $\overline{G(t)}=0$.

Importantly, the formula in Eq. (70) potentially allows for a partial resummation of the perturbative expansion stemming from the formalism introduced in Sec. II A. To illustrate this point, we again consider the driven system in Sec. VI A, for which we find that $\hat{B} \sim \omega$. Substituting $\hat{\mathcal{A}} \rightarrow \hat{A}=$ $\left(\hat{p}_{x}^{2}-\hat{p}_{y}^{2}\right) / 2 m$ and $\omega \hat{\mathcal{B}} \rightarrow \hat{B}=\kappa \hat{x} \hat{y}$ into Eq. (70), and computing the time averages associated with the $\alpha$ pulse sequence (Appendix K), we recover the effective Hamiltonian in Eq. (37). We find that only a few terms in Eq. (70) have a nonzero contribution, and we stress that, in contrast with the result (37) obtained using the formalism of Sec. II A, the present result based on the formula (70) guarantees the convergence of the perturbative expansion. Indeed, one readily verifies that all the terms that have been identified at this order of the computations [Eq. (70)] are of the same order $\Omega \sim \kappa / m \omega$. (See Appendix K for more details.)

\section{B. Adiabatic launching}

In this work, we assume that the periodic modulation that drives the system is launched abruptly at some initial time $t_{i}$. In Sec. III, we demonstrate that the long-time dynamics could strongly depend on this choice (Figs. 1 and 5). Hence, after a long time $t \gg T$, the system "remembers" the initial phase of the modulation, or equivalently, the very first pulse that was activated (e.g., $+\hat{A}$ or $-\hat{B}$ in Fig. 5). This sensitivity to the initial phase constitutes an important issue with regard to experimental implementation, where the phase is controlled with a certain uncertainty. One way to "erase" the memory of the system is to ramp up the modulation $\hat{H}(t)=\hat{H}_{0}+\lambda(t) \hat{V}(t)$, where $\lambda(t)=0 \rightarrow 1$ is 
turned on very smoothly. This "adiabatic launching" will effectively annihilate any effect associated with the initial kick $\hat{K}\left(t_{i}\right)$ in Eq. (15). We observe that the time scales of such memory-eraser ramps depend on the scheme under scrutiny; for example, we find that the ramping time needed to erase the memory in the situation shown in Fig. 5 is of the order of $1 / J$.

Furthermore, the adiabatic launching could be exploited to generate specific target states, although we stress that this strategy is inevitably affected by the micromotion dictated by the operator $\hat{K}\left(t_{f}\right)$ in Eq. (15). Indeed, if one initially prepares the system in the ground state of the static Hamiltonian $\hat{H}_{0}$ and then smoothly ramps up the modulation, the system will eventually oscillate around the (target) ground state of the effective Hamiltonian $\hat{H}_{\text {eff }}$. These micromotion oscillations may be problematic for specific applications, where the stability of the target state particularly matters; we remind readers that the micromotion is inherent to the physics of driven systems, and although it is generally limited in real space, it is typically large in momentum or spin space for the various examples treated in this work (Figs. 4 and 6). In view of applications, this discussion also highlights the difference between two possible targets: (1) reaching a specific state (e.g., the ground state of $\hat{H}_{\text {eff }}$ ) or (2) engineering an effective band structure (also associated with $\hat{H}_{\text {eff }}$ ). This aspect of periodically driven systems is left as an open problem for future works.

Finally, we note that the adiabatic launching is not unique, in the sense that the ramping function $\lambda(t)$ can take arbitrarily many different forms. In particular, one could consider a "sequence-preserving adiabatic" protocol where the function $\lambda(t)$ remains constant during each primitive pulse sequence $\left\{\hat{H}_{0}+\hat{V}_{1}, \ldots, \hat{H}_{0}+\hat{V}_{N}\right\}$ [Eq. (27)]. Let us briefly analyze the time evolution of such a driving scheme, based on the simple two-step sequence $\gamma_{+}=\left\{\hat{H}_{0}+\hat{V}, \hat{H}_{0}-\hat{V}\right\}$, where the + index indicates that the sequence starts with the pulse $+\hat{V}$. Suppose that the system is initially prepared in the ground state $\psi_{0}$ of the static Hamiltonian $\hat{H}_{0}$. At the end of the sequence-preserving ramping process, the state has evolved into $\psi_{0} \rightarrow \psi_{+}$, which is an eigenstate of the effective Hamiltonian $\hat{H}_{+}$associated with the primitive sequence $\gamma_{+}$, namely,

$$
\begin{aligned}
\hat{H}_{+} \psi_{+} & =E \psi_{+}, \quad e^{-i \hat{H}_{+} T}=e^{-i\left(\hat{H}_{0}-\hat{V}\right) T / 2} e^{-i\left(\hat{H}_{0}+\hat{V}\right) T / 2}, \\
\hat{H}_{+} & =\hat{H}_{0}-i \frac{T}{4}\left[\hat{H}_{0}, \hat{V}\right]+\mathcal{O}\left(T^{2}\right) .
\end{aligned}
$$

To estimate the micromotion undergone by the target state $\psi_{+}$after the ramp, we compute its evolution after half a period. We find

$$
\begin{aligned}
e^{-i\left(\hat{H}_{0}+\hat{V}\right) T / 2} \psi_{+} & =\psi_{\text {micro }} \\
\hat{H}_{-} \psi_{\text {micro }} & =E \psi_{\text {micro }} \\
\hat{H}_{-} & =\hat{H}_{0}+i \frac{T}{4}\left[\hat{H}_{0}, \hat{V}\right]+\mathcal{O}\left(T^{2}\right) .
\end{aligned}
$$

Now, suppose that the same driving scheme is performed, but using the alternative sequence $\gamma_{-}=\left\{\hat{H}_{0}-\hat{V}, \hat{H}_{0}+\hat{V}\right\}$. In this case, the target state after the ramp $\psi_{0} \rightarrow \psi_{-}$satisfies

$\hat{H}_{-} \psi_{-}=E \psi_{-}, \quad e^{-i \hat{H}_{-} T}=e^{-i\left(\hat{H}_{0}+\hat{V}\right) T / 2} e^{-i\left(\hat{H}_{0}-\hat{V}\right) T / 2}$.

If the eigenvalue $E$ is not degenerate, i.e., $\psi_{\text {micro }}=\psi_{-}$, we find that the target states associated with the two sequences $\gamma_{ \pm}$are sent into each other $\psi_{+} \leftrightarrow \psi_{-}$through the micromotion [Eqs. (71) and (72)]. If the eigenvalue $E$ is degenerate, which is the case for the ground level of spinorbit-coupled Hamiltonians $[12,13]$, the evolution is nontrivial and should be studied on a case-by-case basis. In both situations, this analysis further highlights the relevance of the micromotion in modulated systems.

\section{Conclusions}

This work was dedicated to the physics of periodically modulated quantum systems, with a view to realizing gauge structures in a wide range of physical contexts.

Our approach was based on the perturbative formalism introduced in Ref. [59], which clearly highlights three relevant notions associated with the driving: the initial kick captured by the operator $\hat{K}\left(t_{i}\right)$, the effective Hamiltonian $\hat{H}_{\text {eff }}$ that rules the long-time dynamics, and the micromotion described by $\hat{K}\left(t_{f}\right)$ (Sec. II A). Based on this perturbative method, we have obtained general formulas and identified diverse driving schemes leading to "nontrivial" effective Hamiltonians, whose characteristics could be useful for the quantum simulation of gauge structures and topological order. In particular, we have discussed the convergence of the perturbative expansion; building on specific examples, we have also presented methods allowing for the partial resummation of infinite series.

This work addresses the general situation where the driving frequency $\omega$ is off resonant with respect to any energy separation present in the problem. However, the effective Hamiltonian method presented here can also be generalized to describe schemes based on resonant driving [78], as recently implemented to generate synthetic magnetic fields in optical lattices [31-34].

We have also mentioned the possibility to switch on the modulations adiabatically; such an adiabatic launching minimizes the effects attributed to the initial kick $\hat{K}\left(t_{i}\right)$ that was shown to be considerable when abruptly launching the driving (Fig. 5). Schemes based on adiabatic launching could also be exploited to reach interesting target states.

This work also aimed to highlight the important role played by the micromotion in periodically driven systems. We have shown that these unavoidable oscillations are typically large in momentum and spin space, for the different examples treated in this work. Taking into account this micromotion is particularly relevant from a detection point of view, noting that various probes are built on (possibly spin-dependent) momentum-distribution imaging. 
Although these results were obtained in the noninteracting regime, they also suggest that dissipation due to interparticle collisions could lead to significant heating in cold-matter systems presenting large micromotion. This heating could be particularly problematic in spin systems, where spindependent micromotion could possibly lead to drastic collision processes. The thermodynamics of driven quantum systems has been recently investigated in Refs. [79-82] (see also Refs. [83,84]). We note that interactions could also be modulated in cold-atom systems, using time-dependent magnetic fields in a Feshbach resonance $[85,86]$.

Finally, we point out that probing interesting effects in cold-matter systems, such as topological order, generally requires us to act on the system with additional potentials $\hat{V}_{\text {probe }}$. For instance, measuring the topologically invariant Chern number in quantum Hall atomic systems [34] can be realized by acting on the cloud with an external force $\hat{V}_{\text {probe }} \sim \hat{x}$ (see also Refs. [87-90]). These additional potentials will contribute to the static Hamiltonian $\hat{H}_{0} \rightarrow \hat{H}_{0}+\hat{V}_{\text {probe }}$, and hence, they will potentially alter the effective Hamiltonian [Eq. (16)] and the corresponding band structure: "Measuring the topological order associated with an effective Hamiltonian may destroy it." This phenomenon will be particularly pronounced when $\hat{V}_{\text {probe }}$ includes Pauli matrices. More generally, adding terms to the static Hamiltonian, either to probe interesting characteristics of the system or to enrich its topological features, should be handled with care. This issue, which is particularly relevant for the field of quantum simulation, is left as an avenue for future works.

\section{ACKNOWLEDGMENTS}

The authors are pleased to acknowledge H. Pichler and F. Gerbier for valuable discussions. They also thank P. Zoller, N. R. Cooper, and S. Nascimbène for stimulating inputs, and G. Juzeliūnas, M. Ueda, and Z. Xu for comments on a preliminary version of this manuscript. N. G. is supported by the Universite Libre de Bruxelles (ULB). This research was also funded by IFRAF and ANR (AGAFON) and by the European Research Council Synergy Grant UQUAM.

\section{APPENDIX A: THE PAUL TRAP}

The Paul trap consists in a particle moving in a modulated harmonic trap. The Hamiltonian is taken in the form

$$
\hat{H}(t)=\hat{H}_{0}+\hat{V} \cos (\omega t)=\frac{\hat{p}^{2}}{2 m}+\frac{1}{2} m \omega_{0}^{2} \hat{x}^{2} \cos (\omega t),
$$

where $\omega=2 \pi / T$ (respectively, $\omega_{0}$ ) denotes the modulation (respectively, harmonic-trap) frequency. It is convenient to write the Schrödinger equation $i \partial_{t} \psi=\hat{H}(t) \psi$ in a moving frame by considering the unitary transformation

$$
\psi=\hat{R} \tilde{\psi}=\exp \left(-i \hat{V} \int_{0}^{t} \cos (\omega \tau) d \tau\right) \tilde{\psi}=e^{-i \sin (\omega t) \hat{V} / \omega} \tilde{\psi}
$$

so that the transformed state satisfies the Schrödinger equation $i \partial_{t} \tilde{\psi}=\tilde{H}(t) \tilde{\psi}$, with the modified Hamiltonian

$$
\begin{aligned}
\tilde{H}(t)=\hat{R}^{\dagger} \hat{H}_{0} \hat{R}= & \hat{H}_{0}-\sin (\omega t) \omega_{0}^{2}(\hat{x} \hat{p}+\hat{p} \hat{x}) / 2 \omega \\
& +m \omega_{0}^{4} \sin ^{2}(\omega t) \hat{x}^{2} / 2 \omega^{2} .
\end{aligned}
$$

Using the Magnus expansion [60] to lowest order, we find that the evolution operator after one period reads

$$
\begin{gathered}
\hat{U}(T) \approx \exp \left(-i \int_{0}^{T} \tilde{H}(\tau) d \tau\right)=\exp \left(-i T \hat{H}_{\mathrm{eff}}\right), \\
\hat{H}_{\mathrm{eff}}=\frac{\hat{p}^{2}}{2 m}+\frac{1}{2} m \Omega^{2} \hat{x}^{2}, \quad \Omega=\omega_{0}^{2} / \sqrt{2} \omega,
\end{gathered}
$$

so that the particle effectively moves in a harmonic trap with frequency $\Omega$. We point out that the nontrivial term in the effective Hamiltonian in Eq. (A4) is second order in the period $T$.

To gain further insight on this result, we consider a classical analysis, which consists in seeking a solution $x(t)$ in the form $x(t)=\bar{x}(t)+\xi(t)$, where $\bar{x}(t)$ evolves slowly and where $\xi(t)$ describes the micromotion. The equations of motion read

$$
\begin{aligned}
m \ddot{\ddot{x}} & =-m \Omega^{2} \bar{x}(t), \\
\xi(t) & =\left(\omega_{0}^{2} / \omega^{2}\right) \cos (\omega t) \bar{x}(t) .
\end{aligned}
$$

Hence, the effective harmonic potential with frequency $\Omega$ that rules the slow motion $\bar{x}(t)$ is equal to the average kinetic energy associated with the micromotion:

$$
\frac{1}{2} m \Omega^{2} \bar{x}^{2}=\frac{1}{2} m\left\langle\dot{\xi}^{2}\right\rangle,
$$

where the angled brackets denote the average over one period. Furthermore, the momentum dispersion in the ground state of the effective Hamiltonian $\hat{H}_{\text {eff }}$ [Eq. (A4)] is $\Delta p=\sqrt{m \Omega}$, which is similar to the momentum associated with the average micromotion $m \dot{\xi}$ over the extension of this ground state. This classical analysis illustrates the important role played by the micromotion in modulated systems.

We now present the first corrections to the result presented in Eq. (A4). The Magnus expansion reads [60] 


$$
\begin{aligned}
\hat{U}(T) & =\exp [-i \mathcal{H}(T)] \\
& =\exp \left(-i\left[\mathcal{H}^{(0)}(T)+\mathcal{H}^{(1)}(T)+\cdots\right]\right), \\
\mathcal{H}^{(0)}(T) & =\int_{0}^{T} \tilde{H}(\tau) d \tau, \\
\mathcal{H}^{(1)}(T) & =-\frac{i}{2} \int_{0}^{T} \int_{0}^{t}[\tilde{H}(t), \tilde{H}(\tau)] d \tau d t,
\end{aligned}
$$

where $\tilde{H}(t)$ is defined in Eq. (A3). The zeroth-order term is given in Eq. (A4), and the first-order corrections read

$\mathcal{H}^{(1)}(T)=4 \gamma T\left(\hat{H}_{0}-\frac{1}{2} m \Omega^{2} \hat{x}^{2}\right), \quad \gamma=\left(\Omega / \omega_{0}\right)^{2}$.

The evolution operator including first-order corrections is finally given by [Eqs. (A7) and (A8)]

$$
\begin{aligned}
\hat{U}(T) & \approx \exp \left(-i T \tilde{H}_{\mathrm{eff}}\right), \\
\tilde{H}_{\mathrm{eff}} & =\frac{\hat{p}^{2}}{2 m}(1+4 \gamma)+\frac{1}{2} m \Omega^{2} \hat{x}^{2}(1-4 \gamma),
\end{aligned}
$$

where we note that the corrections are small $\gamma \ll 1$ for $\omega_{0} \ll \omega$.

Finally, we show how the formalism of Appendix C allows one to recover the result in Eq. (A9). To second order in $(1 / \omega)$, the effective Hamiltonian and kick operators read [Eqs. (C10) and (C11)]

$$
\begin{aligned}
& \hat{H}_{\mathrm{eff}}=\hat{H}_{0}+\frac{1}{4 \omega^{2}}\left[\left[\hat{V}, \hat{H}_{0}\right], \hat{V}\right]+\mathcal{O}\left(1 / \omega^{3}\right), \\
& \hat{K}(0)=\frac{1}{i \omega^{2}}\left[\hat{V}, \hat{H}_{0}\right]+\mathcal{O}\left(1 / \omega^{3}\right),
\end{aligned}
$$

where we use the fact that $V^{(j)}=V^{(-j)}=\hat{V} / 2$ in the single-harmonic case $\hat{H}(t)=\hat{H}_{0}+\hat{V} \cos (\omega t)$. The commutators in Eq. (A10) are readily computed using the operators defined in Eq. (A1), which yields

$$
\begin{aligned}
\hat{H}_{\mathrm{eff}} & =\frac{\hat{p}^{2}}{2 m}+\frac{1}{2} m \Omega^{2} \hat{x}^{2}+\mathcal{O}\left(1 / \omega^{3}\right), \\
\hat{K}(0) & =\gamma(\hat{x} \hat{p}+\hat{p} \hat{x})+\mathcal{O}\left(1 / \omega^{3}\right),
\end{aligned}
$$

where $\Omega$ and $\gamma$ are defined in Eqs. (A4)-(A8). Using Eq. (C5), we obtain the evolution operator after one period

$$
\begin{aligned}
\hat{U}(T) & =e^{-i \hat{K}(0)} e^{-i T \hat{H}_{\mathrm{eff}}} e^{i \hat{K}(0)}=e^{-i T \hat{H}_{\mathrm{eff}}^{T}}, \\
\hat{H}_{\mathrm{eff}}^{\mathcal{T}} & =\frac{\hat{p}^{2}}{2 m}(1+4 \gamma)+\frac{1}{2} m \Omega^{2} \hat{x}^{2}(1-4 \gamma)+\mathcal{O}\left(1 / \omega^{4}\right),
\end{aligned}
$$

such that we recover the result in Eq. (A9).
APPENDIX B: RENORMALIZATION OF THE HOPPING IN MODULATED OPTICAL LATTICES

In this Appendix, we consider a modulated 1D optical lattice, described by the single-particle Hamiltonian

$$
\hat{h}(t)=\hat{p}^{2} / 2 m+V_{\mathrm{OL}}\left[\hat{x}-x_{0}(t)\right],
$$

where the periodic function $x_{0}(t)=x_{0}(t+T)$ is considered to have a zero mean value over one period $T=2 \pi / \omega$, and where $V_{\mathrm{OL}}(\hat{x})$ is the optical-lattice potential. In the absence of driving, the static Hamiltonian $\hat{H}_{0}$ is written in the form of a second-quantized tight-binding Hamiltonian

$$
\hat{H}_{0}=-J\left(\hat{T}+\hat{T}^{\dagger}\right), \quad \hat{T}=\sum_{j} \hat{a}_{j+1}^{\dagger} \hat{a}_{j},
$$

where the operator $\hat{a}_{j}^{\dagger}$ creates a particle at lattice site $x=j a, a$ is the lattice spacing, and $J$ is the hopping-matrix element. The modulated lattice is generally studied in a moving frame, in which case the driving acts through an inertial force

$$
\hat{H}(t)=\hat{H}_{0}+\omega \xi(t) \hat{V}=\hat{H}_{0}+\omega \xi(t) \sum_{j} j \hat{a}_{j}^{\dagger} \hat{a}_{j},
$$

where $\xi(t)=(m a / \omega) \ddot{x}_{0}(t)$. In the following, we set

$$
\xi(t)=\xi_{0} \cos (\omega t),
$$

so that the Hamiltonian reads

$$
\hat{H}(t)=\hat{H}_{0}+\omega \xi_{0} \hat{V} \cos (\omega t) .
$$

Note that the parameter $\kappa$ introduced in Eq. (4) (main text) is given by $\kappa=\omega \xi_{0}$. We also introduce the operator

$$
\hat{H}_{1}=i J\left(\hat{T}-\hat{T}^{\dagger}\right),
$$

which, together with $\hat{H}_{0}$ and $\hat{V}$, form a close set under the action of the commutator

$\left[\hat{H}_{0}, \hat{V}\right]=-i \hat{H}_{1}, \quad\left[\hat{H}_{1}, \hat{V}\right]=i \hat{H}_{0}, \quad\left[\hat{H}_{0}, \hat{H}_{1}\right]=0$.

The latter relations lead to the useful formula

$$
e^{i \gamma \hat{V}} \hat{H}_{0} e^{-i \gamma \hat{V}}=\hat{H}_{0} \cos \gamma-\hat{H}_{1} \sin \gamma,
$$

which is indeed valid for any triple of operators $\left\{\hat{H}_{0}, \hat{H}_{1}, \hat{V}\right\}$ satisfying Eq. (B7); see also Eq. (C7). Following the same procedure as in Appendix A [Eqs. (A2) and (A3)], we consider the unitary transformation

$$
\psi=\hat{R} \tilde{\psi}=e^{-i \xi_{0} \sin (\omega t) \hat{V}} \tilde{\psi}
$$

and the associated modified Hamiltonian 


$$
\begin{aligned}
\tilde{H}(t)= & \hat{R}^{\dagger} \hat{H}_{0} \hat{R}=\hat{H}_{0} \cos \left[\xi_{0} \sin (\omega t)\right] \\
& -\hat{H}_{1} \sin \left[\xi_{0} \sin (\omega t)\right],
\end{aligned}
$$

where we use Eq. (B8). Finally, the operator evolution after one period is given by

$$
\begin{aligned}
\hat{U}(T) & =\exp \left(-i \int_{0}^{T} \tilde{H}(\tau) d \tau\right)=\exp \left(-i T \hat{H}_{\mathrm{eff}}\right), \\
\hat{H}_{\text {eff }} & =\mathcal{J}_{0}\left(\xi_{0}\right) \hat{H}_{0}
\end{aligned}
$$

where we recover the renormalization of the hopping by the Bessel function of the first kind

$$
\mathcal{J}_{0}(x)=\frac{1}{\pi} \int_{0}^{\pi} \cos [x \sin (\tau)] d \tau .
$$

We emphasize that the effective Hamiltonian in Eq. (B9) is exact.

\section{APPENDIX C: GENERAL EXPRESSION FOR THE EFFECTIVE HAMILTONIAN: DERIVATION OF EQS. (16) AND (17)}

We start with the Schrödinger equation

$$
\begin{aligned}
i \partial_{t} \psi(t) & =\hat{H}(t) \psi(t), \\
\hat{H}(t) & =\hat{H}_{0}+\hat{V}(t), \quad \hat{V}(t+T)=\hat{V}(t)
\end{aligned}
$$

and consider the unitary transformation

$$
\phi(t)=\hat{\mathcal{U}}(t) \psi(t)=e^{i \hat{K}(t)} \psi(t) .
$$

The new state $\phi(t)$ satisfies the Schrödinger equation

$$
\begin{aligned}
i \partial_{t} \phi(t) & =\hat{H}_{\mathrm{eff}} \phi(t), \\
\hat{H}_{\mathrm{eff}} & =e^{i \hat{K}(t)} \hat{H}(t) e^{-i \hat{K}(t)}+i\left(\frac{\partial e^{i \hat{K}(t)}}{\partial t}\right) e^{-i \hat{K}(t)},
\end{aligned}
$$

where we introduce the effective Hamiltonian $\hat{H}_{\text {eff }}$. The method of Ref. [59] then consists in constructing the timeindependent effective Hamiltonian $\hat{H}_{\text {eff }}$, by transferring all undesired (time-dependent) terms into the operator $\hat{K}(t)$. The latter has a simple interpretation that becomes obvious when writing the evolution operator

$$
\hat{U}\left(t_{i} \rightarrow t_{f}\right) \psi\left(t_{i}\right)=e^{-i \hat{K}\left(t_{f}\right)} e^{-i \hat{H}_{\text {eff }}\left(t_{f}-t_{i}\right)} e^{i \hat{K}\left(t_{i}\right)} \psi\left(t_{i}\right) .
$$

This expression indicates that the evolution can be split into three parts: (a) an initial kick associated with the operator $\hat{K}\left(t_{i}\right)$, (b) the evolution dictated by the time-independent effective Hamiltonian $\hat{H}_{\text {eff }}$, and (c) a final kick associated with the operator $\hat{K}\left(t_{f}\right)$.

In general, it is not possible to give an analytical expression for the operators $\hat{H}_{\text {eff }}$ and $\hat{K}(t)$ in Eq. (C4). (See, however, Appendix E for an exactly solvable example.) Thus, it is convenient to build these operators perturbatively, by expanding them in powers of the driving period $T=2 \pi / \omega$, which is assumed to be small in the problem. Following Ref. [59], we write

$$
\hat{H}_{\mathrm{eff}}=\sum_{n=0}^{\infty} \frac{1}{\omega^{n}} \hat{H}_{\mathrm{eff}}^{(n)}, \quad \hat{K}=\sum_{n=1}^{\infty} \frac{1}{\omega^{n}} \hat{K}^{(n)}
$$

and consider the expansions

$$
\begin{gathered}
e^{i \hat{K}} \hat{H} e^{-i \hat{K}}=\hat{H}+i[\hat{K}, \hat{H}]-\frac{1}{2}[\hat{K},[\hat{K}, \hat{H}]] \\
-\frac{i}{6}[\hat{K},[\hat{K},[\hat{K}, \hat{H}]]] \ldots \\
\left(\frac{\partial e^{i \hat{K}}}{\partial t}\right) e^{-i \hat{K}}=i \frac{\partial \hat{K}}{\partial t}-\frac{1}{2}\left[\hat{K}, \frac{\partial \hat{K}}{\partial t}\right]-\frac{i}{6}\left[\hat{K},\left[\hat{K}, \frac{\partial \hat{K}}{\partial t}\right]\right] \ldots
\end{gathered}
$$

to determine the operators $\hat{H}_{\text {eff }}$ and $\hat{K}(t)$ at the desired order $\mathcal{O}\left(1 / \omega^{n}\right)$. Note that we further impose that $\hat{K}(t)$ should be periodic $\hat{K}(t)=\hat{K}(t+T)$, with a zero mean value over one period.

We now apply this strategy to the general situation in Eq. (13), where the Hamiltonian $\hat{H}(t)$ of the driven system is given by

$$
\hat{H}(t)=\hat{H}_{0}+\hat{V}(t)=\hat{H}_{0}+\sum_{j=1}^{\infty} \hat{V}^{(j)} e^{i j \omega t}+\hat{V}^{(-j)} e^{-i j \omega t} .
$$

Following the expansion procedure (C6)-(C8) up to second order $\mathcal{O}\left(1 / \omega^{2}\right)$, we obtain the general expressions for the effective Hamiltonian

$$
\begin{aligned}
\hat{H}_{\mathrm{eff}}=\hat{H}_{0}+\frac{1}{\omega} \sum_{j=1}^{\infty} \frac{1}{j}\left[\hat{V}^{(j)}, \hat{V}^{(-j)}\right] & +\frac{1}{2 \omega^{2}} \sum_{j=1}^{\infty} \frac{1}{j^{2}}\left(\left[\left[\hat{V}^{(j)}, \hat{H}_{0}\right], \hat{V}^{(-j)}\right]+\text { H.c. }\right) \\
& +\frac{1}{3 \omega^{2}} \sum_{j, l=1}^{\infty} \frac{1}{j l}\left(\left[\hat{V}^{(j)},\left[\hat{V}^{(l)}, \hat{V}^{(-j-l)}\right]\right]-2\left[\hat{V}^{(j)},\left[\hat{V}^{(-l)}, \hat{V}^{(l-j)}\right]\right]+\text { H.c. }\right)
\end{aligned}
$$

and for the kick operator at time $t$ 


$$
\begin{aligned}
\hat{K}(t)= & \frac{1}{i \omega} \sum_{j=1}^{\infty} \frac{1}{j}\left(\hat{V}^{(j)} e^{i j \omega t}-\hat{V}^{(-j)} e^{-i j \omega t}\right)+\frac{1}{i \omega^{2}} \sum_{j=1}^{\infty} \frac{1}{j^{2}}\left(\left[\hat{V}^{(j)}, \hat{H}_{0}\right] e^{i j \omega t}-\text { H.c. }\right) \\
& +\frac{1}{2 i \omega^{2}} \sum_{j, l=1}^{\infty} \frac{1}{j(j+l)}\left(\left[\hat{V}^{(j)}, \hat{V}^{(l)}\right] e^{i(j+l) \omega t}-\text { H.c. }\right)+\frac{1}{2 i \omega^{2}} \sum_{j \neq l=1}^{\infty} \frac{1}{j(j-l)}\left(\left[\hat{V}^{(j)}, \hat{V}^{(-l)}\right] e^{i(j-l) \omega t}-\text { H.c. }\right) .
\end{aligned}
$$

\section{APPENDIX D: THE TWO-STEP SEQUENCE: DERIVATION OF EQS. (18) AND (19)}

In the simple case of the two-step sequence $\left\{\hat{H}_{0}+\hat{V}, \hat{H}_{0}-\hat{V}\right\}$, the Hamiltonian is given by $\hat{H}(t)=\hat{H}_{0}+f(t) \hat{V}$, where $f(t)$ is the standard squarewave function. Expanding $f(t)$ into its Fourier components, we obtain a simple expression for the $\hat{V}^{(j)}$ operators introduced in Eq. (C9)

$$
\begin{aligned}
\hat{V}^{(j)} & =-2 i \hat{V} / \pi j=\left(\hat{V}^{(-j)}\right)^{\dagger} \text { for } j \text { odd } \\
& =0 \text { otherwise. }
\end{aligned}
$$

Inserting these operators into Eqs. (16) and (17) yields the result

$$
\begin{array}{r}
\hat{H}_{\text {eff }}=\hat{H}_{0}+\frac{\pi^{2}}{24 \omega^{2}}\left[\left[\hat{V}, \hat{H}_{0}\right], \hat{V}\right]+\mathcal{O}\left(1 / \omega^{3}\right), \\
\hat{K}(t)=-\frac{\pi}{2 \omega} \hat{V}+|t| \hat{V}+\mathcal{O}\left(1 / \omega^{2}\right) \text { for } t \in\left[-\frac{T}{2}, \frac{T}{2}\right] .
\end{array}
$$

To derive Eq. (D2), we use the formula

$$
\sum_{j=1}^{\infty} 1 /(2 j-1)^{4}=\pi^{4} / 96 .
$$

To derive Eq. (D3), we use the formula

$$
\sum_{j=1}^{\infty} \frac{\cos }{(2 j-1)^{2}}=\frac{\pi}{4}\left(\frac{\pi}{2}-|x|\right),
$$

which is valid for $-\pi \leq x \leq \pi$; see Ref. [91].

\section{APPENDIX E: THE MODULATED OPTICAL LATTICE REVISITED}

In this Appendix, we illustrate how the method described in Appendix $\mathrm{C}$ can be used in a nonperturbative manner, based on the modulated-optical-lattice problem treated in Appendix B. The present analysis allows us to recover the well-known renormalization of the hopping amplitude by a Bessel function; see Eq. (B9).

As in Appendix B, we start with the Hamiltonian

$$
\hat{H}(t)=\hat{H}_{0}+m a \ddot{x}_{0}(t) \hat{V}=\hat{H}_{0}+\omega \xi(t) \sum_{j} j \hat{a}_{j}^{\dagger} \hat{a}_{j},
$$

where the tight-binding Hamiltonians $\hat{H}_{0}$ and $\hat{V}$ are defined in Eqs. (B2) and (B3). Following the strategy described in Eqs. (C2)-(C4), we look for a unitary transformation $\hat{\mathcal{U}}(t)$, such that

$$
\hat{H}_{\mathrm{eff}}=\hat{\mathcal{U}}(t) \hat{H}(t) \hat{\mathcal{U}}^{\dagger}(t)+i\left(\frac{\partial \hat{\mathcal{U}}(t)}{\partial t}\right) \hat{\mathcal{U}}^{\dagger}(t)
$$

defines a time-independent effective Hamiltonian. The unknown unitary transformation is taken in the form

$$
\hat{\mathcal{U}}(t)=e^{i\left[\alpha(t) \hat{H}_{0}+\beta(t) \hat{H}_{1}\right]} e^{i \gamma(t) \hat{V}},
$$

where the operator $\hat{H}_{1}$ is introduced in Eq. (B6).

Using the identities (B7) and (B8) together with the ansatz (E3), we find that the effective Hamiltonian in Eq. (E2) takes the simple form

$$
\begin{aligned}
\hat{H}_{\mathrm{eff}}= & \hat{H}_{0}(\cos \gamma-\omega \xi \beta+\beta \dot{\gamma}-\dot{\alpha})+\hat{V}(\omega \xi-\dot{\gamma}) \\
& +\hat{H}_{1}(-\sin \gamma+\omega \xi \alpha-\alpha \dot{\gamma}-\dot{\beta}),
\end{aligned}
$$

where the three time-periodic functions $\alpha, \beta$, and $\gamma$ are still to be determined. Imposing that $\hat{H}_{\text {eff }}$ should be time independent,

$$
\hat{H}_{\text {eff }}=c_{1} \hat{H}_{0}+c_{2} \hat{H}_{1}+c_{0} \hat{V}
$$

leads to the relations

$$
\begin{aligned}
& \alpha(t)=\alpha_{0}+\int_{0}^{t} \cos \left[\omega \int_{0}^{t^{\prime}} \xi\left(t^{\prime \prime}\right) d t^{\prime \prime}-\gamma_{0}\right] d t^{\prime}-c_{1} t, \\
& \beta(t)=\beta_{0}+\int_{0}^{t} \sin \left[\omega \int_{0}^{t^{\prime}} \xi\left(t^{\prime \prime}\right) d t^{\prime \prime}-\gamma_{0}\right] d t^{\prime}-c_{2} t, \\
& \gamma(t)=\gamma_{0}+\omega \int_{0}^{t} \xi\left(t^{\prime}\right) d t^{\prime}-c_{0} t,
\end{aligned}
$$

where we introduce constants of integration. The time periodicity of $\gamma(t)$ is satisfied by setting $c_{0}=0$, while imposing the periodicity of the two other functions $\alpha$ and $\beta$ requires further developments. For the sake of clarity, let us consider the driving $x_{0}(t)=\zeta \cos (\omega t+\phi)$, such that

$$
\xi(t)=\xi_{0} \cos (\omega t+\phi), \quad \xi_{0}=-m a \omega \zeta .
$$

In this case, the time periodicity of $\alpha(t)$ and $\beta(t)$ in Eq. (E4) is satisfied for

$$
c_{1}=\mathcal{J}_{0}\left(\xi_{0}\right) \cos \gamma_{1}, \quad c_{2}=-\mathcal{J}_{0}\left(\xi_{0}\right) \sin \gamma_{1},
$$


where $\gamma_{1}=\gamma_{0}-\xi_{0} \sin \phi$, and where $\mathcal{J}_{0}\left(\xi_{0}\right)$ is the Bessel function of the first kind in Eq. (B10). The corresponding effective Hamiltonian reads

$$
\hat{H}_{\text {eff }}=\mathcal{J}_{0}\left(\xi_{0}\right)\left(\hat{H}_{0} \cos \gamma_{1}-\hat{H}_{1} \sin \gamma_{1}\right),
$$

where we note that the operators $\hat{H}_{0,1}$ are related by a gauge transformation.

Next, we rewrite the evolution operator in Eq. (E3) in the more usual form [Eq. (C2)]

$$
\begin{aligned}
\hat{\mathcal{U}}(t)= & e^{i \hat{K}(t)}, \\
\hat{K}(t)= & \hat{H}_{0}\left\{\alpha(t) \mathfrak{c}_{1}+\beta(t) \mathfrak{c}_{2}\right\}+\hat{H}_{1}\left\{\beta(t) \mathfrak{c}_{1}-\alpha(t) \mathfrak{c}_{2}\right\} \\
& +\gamma(t) \hat{V},
\end{aligned}
$$

where we use the commutators in Eq. (B7), and where the constants $\mathfrak{c}_{1,2}$ can be deduced from the $\mathrm{BCH}$ Trotter expansion in Eq. (9). From Eq. (E8), we find that the function $\gamma(t)$ in Eq. (E4) should necessarily have a zero mean value over one period to guarantee that the kick operator $\hat{K}(t)$ satisfies this same constraint. This latter condition sets $\gamma_{1}=0$, which together with Eq. (E7) eventually leads to the familiar renormalization of the hopping amplitude

$$
\hat{H}_{\text {eff }}=\mathcal{J}_{0}\left(\xi_{0}\right) \hat{H}_{0}, \quad \xi_{0}=-m a \omega \zeta,
$$

which reproduces the result presented in Eq. (B9).

The remaining constants $\alpha_{0}, \beta_{0}$, and $\gamma_{0}$ define the initial operator $\hat{\mathcal{U}}(0)$ and thus complete the determination of the unitary transformation $\hat{\mathcal{U}}(t)$. The condition $\gamma_{1}=0$ implies $\gamma_{0}=\xi_{0} \sin \phi$, such that setting $\alpha_{0}=\beta_{0}$ yields

$$
\hat{U}(0)=e^{i \xi_{0} \sin \phi \hat{V}},
$$

which determines the kick operator $\hat{K}(0)=\xi_{0} \sin \phi \hat{V}$. This latter result illustrates the fact that the initial phase of the lattice modulation $\phi$ has an impact on the initial kick given to the system but has no consequence on the effective Hamiltonian [19].

\section{APPENDIX F: $N$-STEP SEQUENCE: DERIVATION OF THE GENERAL EXPRESSION IN EQ. (31)}

We derive the general expressions in Eq. (31) for the effective Hamiltonian $\hat{H}_{\text {eff }}$ and initial-kick operator $\hat{K}(0)$, in the general case presented in Sec. IV, where the system is characterized by the repeated pulse sequence

$\gamma_{N}=\left\{\hat{H}_{0}+\hat{V}_{1}, \hat{H}_{0}+\hat{V}_{2}, \hat{H}_{0}+\hat{V}_{3}, \ldots, \hat{H}_{0}+\hat{V}_{N}\right\}$,

with $N$ an arbitrary integer. We write the Hamiltonian as

$$
\hat{H}(t)=\hat{H}_{0}+\sum_{j \neq 0} \hat{V}^{(j)} e^{i \omega j t},
$$

$$
\hat{V}^{(j)}=\frac{1}{2 \pi i} \sum_{m=1}^{N} \frac{1}{j} e^{-i 2 \pi j m / N}\left(e^{i j(2 \pi / N)}-1\right) \hat{V}_{m},
$$

where we use the Fourier series in Eq. (28). Applying Eqs. (16) and (17), and presently restricting ourselves to the first-order terms, yields

$$
\begin{aligned}
\hat{H}_{\mathrm{eff}} & =\hat{H}_{0}+\frac{i}{2 \pi^{2} \omega} \sum_{m, n=1}^{N}\left[\hat{V}_{m}, \hat{V}_{n}\right] \mathcal{C}_{m, n}+\mathcal{O}\left(1 / \omega^{2}\right), \\
\hat{K}(0) & =-\frac{2}{\pi \omega} \sum_{m=1}^{N} \hat{V}_{m} \tilde{\mathcal{C}}_{m}+\mathcal{O}\left(1 / \omega^{2}\right), \\
\mathcal{C}_{m, n} & =\sum_{j=1}^{\infty} \frac{1}{j^{3}} \sin \left(\frac{2 \pi j}{N}(n-m)\right)\left[1-\cos \left(\frac{2 \pi j}{N}\right)\right], \\
\tilde{\mathcal{C}}_{m} & =\sum_{j=1}^{\infty} \frac{1}{j^{2}} \sin \left(\frac{\pi j}{N}(2 m-1)\right) \sin \left(\frac{\pi j}{N}\right) .
\end{aligned}
$$

These expressions can be simplified using the formulas [91]

$$
\begin{array}{ll}
\sum_{k=1}^{\infty} \frac{\sin k x}{k^{3}}=\frac{\pi^{2} x}{6}-\frac{\pi x^{2}}{4}+\frac{\pi^{3}}{12}, & 0 \leq x \leq 2 \pi, \\
\sum_{k=1}^{\infty} \frac{\cos k x}{k^{2}}=\frac{\pi^{2}}{6}-\frac{\pi x}{2}+\frac{\pi^{2}}{4}, & 0 \leq x \leq 2 \pi,
\end{array}
$$

yielding the first-order terms presented in Eqs. (31).

A similar calculation allows us to evaluate the effective Hamiltonian's second-order terms. For the sake of simplicity, we restrict ourselves to the second-order term

$$
\hat{H}_{\mathrm{eff}}^{(2)}=\frac{1}{2 \omega^{2}} \sum_{j=1}^{\infty} \frac{1}{j^{2}}\left(\left[\left[\hat{V}^{(j)}, \hat{H}_{0}\right], \hat{V}^{(-j)}\right]+\text { H.c. }\right),
$$

noticing that the harmonic-mixing terms [i.e., the second line in Eq. (C10)] do not contribute for the sequences treated in this work. Using the expansion in Eq. (F3) for the $V^{(j)}$ operators yields

$$
\begin{aligned}
& \hat{H}_{\mathrm{eff}}^{(2)}=\frac{1}{2 \pi^{2} \omega^{2}} \sum_{m<n=2}^{N} \hat{C}_{m, n} \mathcal{D}_{m, n}+\frac{1}{4 \pi^{2} \omega^{2}} \sum_{m=1}^{N} \hat{C}_{m, m} \mathcal{D}_{m, m}, \\
& \mathcal{D}_{m, n}=\sum_{j=1}^{\infty} \frac{1}{j^{4}} \cos \left(\frac{2 \pi j}{N}(n-m)\right)\left[1-\cos \left(\frac{2 \pi j}{N}\right)\right],
\end{aligned}
$$

where we introduce the commutators

$$
\hat{C}_{m, n}=\left[\left[\hat{V}_{m}, \hat{H}_{0}\right], \hat{V}_{n}\right]+\left[\left[\hat{V}_{n}, \hat{H}_{0}\right], \hat{V}_{m}\right] .
$$

Using the formula [91] 


$$
\sum_{k=1}^{\infty} \frac{\cos k x}{k^{4}}=\frac{\pi^{4}}{90}-\frac{\pi^{2} x^{2}}{12}+\frac{\pi x^{3}}{12}-\frac{x^{4}}{48}, \quad 0 \leq x \leq 2 \pi
$$

one finds simple expressions for the coefficients in Eq. (F6):

$$
\begin{aligned}
& \mathcal{D}_{m, n}=\frac{\pi^{4}}{3 N^{4}}\left[1+N^{2}-6 N(n-m)+6(n-m)^{2}\right](\text { for } m<n), \\
& \mathcal{D}_{m, m}=\frac{\pi^{4}}{3 N^{4}}(N-1)^{2}(\text { for } m=n) .
\end{aligned}
$$

Inserting these coefficients into Eq. (F6) yields the final result presented in Eq. (31).

\section{APPENDIX G: EFFECTIVE HAMILTONIANS FOR GENERAL SEQUENCES WITH $N=3,4$ DIFFERENT STEPS}

In this Appendix, we apply the expression in Eq. (31) to general sequences with $N=3,4$ different steps. The case $N=2$ is fully treated in the main text and in Appendix D.

\section{The case $N=3$}

We first consider a general sequence with three different repeated steps: $\gamma_{3}=\left\{\hat{H}_{0}+\hat{V}_{1}, \hat{H}_{0}+\hat{V}_{2}, \hat{H}_{0}+\hat{V}_{3}\right\}$. In this case, the expressions in Eq. (31) yield

$$
\begin{aligned}
\hat{H}_{\mathrm{eff}}= & \hat{H}_{0}+\frac{i \pi}{27 \omega}\left(\left[\hat{V}_{1}, \hat{V}_{2}\right]+\left[\hat{V}_{2}, \hat{V}_{3}\right]+\left[\hat{V}_{3}, \hat{V}_{1}\right]\right) \\
& +\frac{\pi^{2}}{243 \omega^{2}}\left(\hat{C}_{1,1}+\hat{C}_{2,2}+\hat{C}_{3,3}-\hat{C}_{1,2}-\hat{C}_{2,3}-\hat{C}_{1,3}\right) \\
& +\mathcal{O}\left(1 / \omega^{3}\right) \\
\hat{K}(0)= & \frac{2 \pi}{9 \omega}\left(-\hat{V}_{1}+\hat{V}_{3}\right)+\mathcal{O}\left(1 / \omega^{2}\right),
\end{aligned}
$$

where the commutators $\hat{C}_{m, n}$ are defined in Eq. (F7). In contrast with the case $N=2$, a proper choice of the operators $\hat{H}_{0}$ and $\hat{V}_{1,2,3}$ can potentially lead to nontrivial effects that are first order in $(1 / \omega)$. This scenario was considered by Kitagawa et al. in Ref. [52] to realize the Haldane model [38] in a honeycomb lattice with pulsed hopping terms.

\section{The case $N=4$}

We now consider the case of four-step sequences, which are principally explored in this work, and which have also been the basis of several proposals $[15,25,26]$. In this case, the expressions in Eq. (31) yield

$$
\begin{aligned}
\hat{H}_{\mathrm{eff}}= & \hat{H}_{0}+\frac{i \pi}{32 \omega}\left(\left[\hat{V}_{1}, \hat{V}_{2}\right]+\left[\hat{V}_{2}, \hat{V}_{3}\right]+\left[\hat{V}_{3}, \hat{V}_{4}\right]+\left[\hat{V}_{4}, \hat{V}_{1}\right]\right) \\
& +\frac{\pi^{2}}{256 \omega^{2}}\left[\frac{3}{4}\left(\hat{C}_{1,1}+\hat{C}_{2,2}+\hat{C}_{3,3}+\hat{C}_{4,4}\right)\right. \\
& \left.-\frac{1}{6}\left(\hat{C}_{1,2}+\hat{C}_{2,3}+\hat{C}_{3,4}+\hat{C}_{1,4}\right)-\frac{7}{6}\left(\hat{C}_{1,3}+\hat{C}_{2,4}\right)\right] \\
& +\mathcal{O}\left(1 / \omega^{3}\right), \\
\hat{K}(0)= & \frac{\pi}{16 \omega}\left(-3 \hat{V}_{1}-\hat{V}_{2}+\hat{V}_{3}+3 \hat{V}_{4}\right)+\mathcal{O}\left(1 / \omega^{2}\right), \quad(\mathrm{G} 2)
\end{aligned}
$$

where we note the absence of commutators $\left[\hat{V}_{1}, \hat{V}_{3}\right]$ and $\left[\hat{V}_{2}, \hat{V}_{4}\right]$ in $\hat{H}_{\text {eff }}$, and where the commutators $\hat{C}_{m, n}$ are defined in Eq. (F7). As for the case $N=3$, a proper choice of the operators $\hat{H}_{0}$ and $\hat{V}_{1,2,3,4}$ can potentially lead to nontrivial effects that are first order in $(1 / \omega)$.

Let us now focus on the $\alpha$ sequence in Eq. (32), introduced in Sec. VA.

First, we note that the third-order corrections are easily obtained when the $\alpha$ sequence in Eq. (32) is approximated by the smooth single-harmonic driving $\hat{V}(t)=\hat{A} \cos (\omega t)+$ $\hat{B} \sin (\omega t)$. Pushing the perturbative expansion of Appendix $\mathrm{C}$ to the next order, we find

$$
\begin{aligned}
\hat{H}_{\mathrm{eff}}= & \hat{H}_{0}+\frac{i}{2 \omega}[\hat{A}, \hat{B}]+\frac{1}{4 \omega^{2}}\left(\left[\left[\hat{A}, \hat{H}_{0}\right], \hat{A}\right]+\left[\left[\hat{B}, \hat{H}_{0}\right], \hat{B}\right]\right) \\
& +\frac{i}{4 \omega^{3}}\left(\left[\hat{A},\left[\left[\hat{B}, \hat{H}_{0}\right], \hat{H}_{0}\right]\right]-\left[\hat{B},\left[\left[\hat{A}, \hat{H}_{0}\right], \hat{H}_{0}\right]\right]\right) \\
& \left.+\frac{i}{16 \omega^{3}}([[\hat{A},[\hat{A}, \hat{B}]], \hat{A}]+[[\hat{B},[\hat{A}, \hat{B}]], \hat{B})]\right) \\
& +\mathcal{O}\left(1 / \omega^{4}\right) .
\end{aligned}
$$

The third-order corrections associated with the original square-wave sequence $\alpha$ [Eq. (32)] can also be evaluated through the alternative perturbative method developed in Appendix K, reading [Eq. (K9)]

$$
\hat{H}_{\mathrm{eff}}^{(3)} / \omega^{3}=+\frac{i \pi^{3}}{768 \omega^{3}}[[\hat{B},[\hat{A}, \hat{B}]], \hat{B}],
$$

where we note that $\pi^{3} / 768 \approx 1 / 16$, in agreement with Eq. (G3). Note that this method disregards the terms

$$
\left[\hat{A},\left[\left[\hat{B}, \hat{H}_{0}\right], \hat{H}_{0}\right]\right], \quad\left[\hat{B},\left[\left[\hat{A}, \hat{H}_{0}\right], \hat{H}_{0}\right]\right], \quad[[\hat{A},[\hat{A}, \hat{B}]], \hat{A}]
$$

at this order of the computation; indeed, the alternative approach implicitly assumes that these terms will contribute to higher orders in $1 / \omega$ (Appendix K). However, we note that the expression in Eq. (G4) applies to the SOC scheme analyzed in Sec. VII B 1, for which the neglected terms in Eq. (G5) all vanish identically. The effects 
associated with the third-order corrections (G4) are illustrated for this specific scheme in Fig. 4.

\section{APPENDIX H: TIGHT-BINDING OPERATORS IN 2D LATTICE SYSTEMS AND USEFUL COMMUTATORS}

In this Appendix, we set a dictionary that translates common operators defined in the bulk $(\hat{x}, \hat{p}, \ldots)$ into their lattice analogues. Here, the lattice framework is treated using a single-band tight-binding approximation. We also provide commutators of these operators, which are useful for the calculations presented in Secs. VI and VII.

In a single-band tight-binding description, the hopping Hamiltonian is taken in the form

$$
\begin{aligned}
\hat{H}_{0} & =\hat{T}_{x}+\hat{T}_{y}, \\
& =-J \sum_{m, n} \hat{a}_{m+1, n}^{\dagger} \hat{a}_{m, n}+\hat{a}_{m, n+1}^{\dagger} \hat{a}_{m, n}+\text { H.c. }
\end{aligned}
$$

where the operators $\hat{T}_{x, y}$ denote hopping along the $(x, y)$ directions with amplitude $J, \hat{a}_{m, n}^{\dagger}$ creates a particle at lattice site $\boldsymbol{x}=(m a, n a)$, and $a$ is the lattice spacing. The indices $(m, n)$ are integers. If we take the continuum limit $a \rightarrow 0$ (or equivalently, if we expand the momentum around the bottom of the band), we recover the usual kinetic energy term

$$
\hat{T}_{x}+\hat{T}_{y} \equiv \frac{1}{2 m^{*}}\left(\hat{p}_{x}^{2}+\hat{p}_{y}^{2}\right), \quad m^{*}=1 /\left(2 J a^{2}\right),
$$

where we introduce the effective mass $m^{*}$. We exploit this bulk-lattice analogy and use the following notation to denote lattice operators:

$$
\frac{\bar{p}_{x}^{2}}{2 m^{*}} \equiv \hat{T}_{x}=-J \sum_{m, n} \hat{a}_{m+1, n}^{\dagger} \hat{a}_{m, n}+\text { H.c. }
$$

\section{One-component lattices}

Table I defines all the lattice operators used in Sec. VI.

\section{TABLE I.}

\begin{tabular}{lc}
\hline \hline Symbol & Tight-binding operator \\
\hline $\bar{p}_{x}^{2} / 2 m^{*}$ & $-J \sum_{m, n} \hat{a}_{m+1, n}^{\dagger} \hat{a}_{m, n}+$ H.c. \\
$\bar{p}_{y}^{2} / 2 m^{*}$ & $-J \sum_{m, n} \hat{a}_{m, n+1}^{\dagger} \hat{a}_{m, n}+$ H.c. \\
$\bar{x}$ & $a \sum_{m, n} m \hat{a}_{m, n}^{\dagger} \hat{a}_{m, n}$ \\
$\bar{x} \bar{y}$ & $a^{2} \sum_{m, n} m n \hat{a}_{m, n}^{\dagger} \hat{a}_{m, n}$ \\
$\bar{p}_{x}$ & $i / 2 a \sum_{m, n} \hat{a}_{m+1, n}^{\dagger} \hat{a}_{m, n}-$ H.c. \\
$\bar{L}_{z}=\bar{x} \bar{p}_{y}-\bar{y} \bar{p}_{x}$ & $i / 2 \sum_{m, n} m \hat{a}_{m, n+1}^{\dagger} \hat{a}_{m, n}-n \hat{a}_{m+1, n}^{\dagger} \hat{a}_{m, n}-$ H.c. \\
$\bar{x}^{2}+\bar{y}^{2}$ & $a^{2} / 2 \sum_{m, n} n^{2} \hat{a}_{m+1, n}^{\dagger} \hat{a}_{m, n}+m^{2} \hat{a}_{m, n+1}^{\dagger} \hat{a}_{m, n}+$ H.c. \\
\hline \hline
\end{tabular}

TABLE II.

\begin{tabular}{ll}
\hline \hline Commutator & \multicolumn{1}{c}{ Result } \\
\hline$\left[\bar{p}_{x}^{2} / 2 m^{*}-\bar{p}_{y}^{2} / 2 m^{*}, \bar{x} \bar{y}\right]$ & $=2 i J a^{2} \bar{L}_{z}=i \bar{L}_{z} / m^{*}$ \\
{$\left[\bar{p}_{x}^{2} / 2 m^{*}+\bar{p}_{y}^{2} / 2 m^{*}, \bar{x} \bar{y}\right]$} & $=-2 i J a^{2}\left(\bar{x} \bar{p}_{y}+\bar{y} \bar{p}_{x}\right)$ \\
$i\left[\bar{x} \bar{p}_{y}+\bar{y} \bar{p}_{x}, \bar{x} \bar{y}\right]$ & $=\bar{x}^{2}+\bar{y}^{2}$ \\
{$\left[\bar{p}_{\mu}^{2} / 2 m^{*}, \bar{\mu}\right], \mu=x, y$} & $=-i \bar{p}_{\mu} / m^{*}$ \\
{$\left[\bar{p}_{\mu}, \bar{\mu}\right] / a^{2} m^{*}, \mu=x, y$} & $=i \bar{p}_{\mu}^{2} / 2 m^{*}$ \\
\hline \hline
\end{tabular}

Table II presents the nonzero commutators required to evaluate $[\hat{A}, \hat{B}]$ and $\left[\left[\hat{H}_{0}, \hat{B}\right], \hat{B}\right]$ in Sec. VI, using the lattice operators defined in Table I.

In particular, setting

$\hat{H}_{0}=\bar{p}_{\mu}^{2} / 2 m^{*}, \quad \hat{V}=\bar{\mu} / a, \quad \hat{H}_{1}=\bar{p}_{\mu} / a m^{*}, \quad \mu=x, y$, we recover the cyclic relations Eqs. (B7), such that Eq. (B8) yields

$e^{i \gamma \bar{\mu} / a}\left(\bar{p}_{\mu}^{2} / 2 m^{*}\right) e^{-i \gamma \bar{\mu} / a}=\left(\bar{p}_{\mu}^{2} / 2 m^{*}\right) \cos \gamma-\left(\bar{p}_{\mu} / a m^{*}\right) \sin \gamma$.

\section{Two-component lattices}

In order to set the notations, we write the two-component tight-binding Hamiltonian in the form

$$
\begin{aligned}
\hat{H}_{0} & =\hat{T}_{x}+\hat{T}_{y} \\
& =-J \sum_{m, n} \hat{\Psi}_{m+1, n}^{\dagger} \hat{\Psi}_{m, n}+\hat{\Psi}_{m, n+1}^{\dagger} \hat{\Psi}_{m, n}+\text { H.c., }
\end{aligned}
$$

where $\Psi_{m, n}^{\dagger}=\left(\hat{a}_{m, n}^{\dagger}, \hat{b}_{m, n}^{\dagger}\right)$ contains the operators that create a particle at lattice site $\boldsymbol{x}=(m a, n a)$ in the state $\sigma= \pm$. Table III defines all the lattice operators used in Sec. VII.

Table IV presents the nonzero commutators required to evaluate the effective Hamiltonians in Sec. VII, using the lattice operators defined in Table III.

TABLE III.

\begin{tabular}{ll}
\hline \hline Symbol & \multicolumn{1}{c}{ Tight-binding operator } \\
\hline $\bar{p}_{x}^{2} / 2 m^{*}$ & $-J \sum_{m, n} \hat{\Psi}_{m+1, n}^{\dagger} \hat{\Psi}_{m, n}+$ H.c. \\
$\bar{p}_{y}^{2} / 2 m^{*}$ & $-J \sum_{m, n} \hat{\Psi}_{m, n+1}^{\dagger} \hat{\Psi}_{m, n}+$ H.c. \\
$\bar{x} \hat{\sigma}_{x}$ & $a \sum_{m, n} m \hat{\Psi}_{m, n}^{\dagger} \hat{\sigma}_{x} \hat{\Psi}_{m, n}$ \\
$\bar{y} \hat{\sigma}_{y}$ & $a \sum_{m, n} n \hat{\Psi}_{m, n}^{\dagger} \hat{\sigma}_{y} \hat{\Psi}_{m, n}$ \\
$\bar{p}_{x} \hat{\sigma}_{x}$ & $i / 2 a \sum_{m, n} \hat{\Psi}_{m+1, n}^{\dagger} \hat{\sigma}_{x} \hat{\Psi}_{m, n}-$ H.c. \\
$\bar{p}_{y} \hat{\sigma}_{y}$ & $i / 2 a \sum_{m, n} \hat{\Psi}_{m, n+1}^{\dagger} \hat{\sigma}_{y} \hat{\Psi}_{m, n}-$ H.c. \\
$\bar{L}_{z} \hat{\sigma}_{z}=\left(\bar{x} \bar{p}_{y}-\bar{y} \bar{p}_{x}\right) \hat{\sigma}_{z}$ & $i / 2 \sum_{m, n}\left(m \hat{\Psi}_{m, n+1}^{\dagger}-n \hat{\Psi}_{m+1, n}^{\dagger}\right) \hat{\sigma}_{z} \hat{\Psi}_{m, n}$ \\
& - H.c. \\
$\left(\bar{x} \bar{p}_{y}+\bar{y} \bar{p}_{x}\right) \hat{\sigma}_{z}$ & $i / 2 \sum_{m, n}\left(m \hat{\Psi}_{m, n+1}^{\dagger}+n \hat{\Psi}_{m+1, n}^{\dagger}\right) \hat{\sigma}_{z} \hat{\Psi}_{m, n}$ \\
& - H.c. \\
\hline \hline
\end{tabular}


TABLE IV.

\begin{tabular}{|c|c|}
\hline Commutator & Result \\
\hline$\overline{\left[\bar{p}_{x}^{2} / 2 m^{*}, \bar{x} \hat{\sigma}_{x}\right]}$ & $=\left(-i / m^{*}\right) \bar{p}_{x} \hat{\sigma}_{x}$ \\
\hline$\left[\bar{p}_{x} \hat{\sigma}_{x}, \bar{x} \hat{\sigma}_{x}\right]$ & $=\left(i a^{2} / 2\right) \bar{p}_{x}^{2}$ \\
\hline$\rightarrow\left[\left[\bar{p}_{x}^{2} / 2 m^{*}, \bar{x} \hat{\sigma}_{x}\right], \bar{x} \hat{\sigma}_{x}\right] / a^{2}$ & $=\bar{p}_{x}^{2} / 2 m^{*}$ \\
\hline$\left[\bar{p}_{y}^{2} / 2 m^{*}, \bar{y} \hat{\sigma}_{y}\right]$ & $=\left(-i / m^{*}\right) \bar{p}_{y} \hat{\sigma}_{y}$ \\
\hline$\left[\bar{p}_{y} \hat{\sigma}_{y}, \bar{y} \hat{\sigma}_{y}\right]$ & $=\left(i a^{2} / 2\right) \hat{p}_{y}^{2}$ \\
\hline$\rightarrow\left[\left[\bar{p}_{y}^{2} / 2 m^{*}, \bar{y} \hat{\sigma}_{y}\right], \bar{y} \hat{\sigma}_{y}\right] / a^{2}$ & $=\bar{p}_{y}^{2} / 2 m^{*}$ \\
\hline$\left[\bar{p}_{x} \hat{\sigma}_{x}, \bar{y} \hat{\sigma}_{y}\right]+\left[\bar{p}_{y} \hat{\sigma}_{y}, \bar{x} \hat{\sigma}_{x}\right]$ & $=-2 i \bar{L}_{z} \hat{\sigma}_{z}$ \\
\hline$\left[\bar{p}_{y} \hat{\sigma}_{y}, \bar{x} \hat{\sigma}_{x}\right]-\left[\bar{p}_{x} \hat{\sigma}_{x}, \bar{y} \hat{\sigma}_{y}\right]$ & $=-2 i\left(\bar{x} \bar{p}_{y}+\bar{y} \bar{p}_{x}\right) \hat{\sigma}_{z}$ \\
\hline
\end{tabular}

Moreover, it is useful to note the cyclic conditions [see also Eq. (B7)]

$\hat{H}_{0}=\bar{p}_{\mu}^{2} / 2 m^{*}, \quad \hat{V}=\bar{\mu} \hat{\sigma}_{\mu} / a, \quad \hat{H}_{1}=\bar{p}_{\mu} \hat{\sigma}_{\mu} / a m^{*}, \quad \mu=x, y$,

$\left[\hat{H}_{0}, \hat{V}\right]=-i \hat{H}_{1}, \quad\left[\hat{H}_{1}, \hat{V}\right]=i \hat{H}_{0}, \quad\left[\hat{H}_{0}, \hat{H}_{1}\right]=0$.

Using Eqs. (H5) and (B8) yields the useful formulas

$$
\begin{aligned}
e^{i \gamma \bar{\mu} \hat{\sigma}_{\mu} / a}\left(\frac{\bar{p}_{\mu}^{2}}{2 m^{*}}\right) e^{-i \gamma \bar{\mu} \hat{\sigma}_{\mu} / a} & =\frac{\bar{p}_{\mu}^{2}}{2 m^{*}} \cos \gamma-\frac{\bar{p}_{\mu} \hat{\sigma}_{\mu}}{a m^{*}} \sin \gamma, \\
e^{i \gamma \bar{\mu} \hat{\sigma}_{\mu} / a}\left(\frac{\bar{p}_{\mu} \hat{\sigma}_{\mu}}{a m^{*}}\right) e^{-i \gamma \bar{\mu} \hat{\sigma}_{\mu} / a} & =\frac{\bar{p}_{\mu} \hat{\sigma}_{\mu}}{a m^{*}} \cos \gamma-\frac{\bar{p}_{\mu}^{2}}{2 m^{*}} \sin \gamma,
\end{aligned}
$$

which are used several times in this work.

\section{APPENDIX I: EXACT TREATMENT OF THE XA SCHEME}

In this Appendix, we derive the effective Hamiltonians in Eqs. (60) and (61), which are associated with the XA scheme.

\section{Without the lattice}

Following Refs. [25,26], it is convenient to partition the time-evolution operator associated with the XA sequence $(55)$ as $\hat{U}(T)=\hat{U}_{y} \hat{U}_{x}$, where the two subsequences read

$$
\hat{U}_{\mu}=e^{-i\left(\hat{H}_{0}-\kappa \hat{\mu} \hat{\sigma}_{\mu}\right) T / 4} e^{-i\left(\hat{H}_{0}+\kappa \hat{\mu} \hat{\sigma}_{\mu}\right) T / 4}, \quad \mu=x, y .
$$

We first focus on the subsequence characterized by the evolution operator $\hat{U}_{x}$ and perform a unitary transformation in the same spirit as in Eq. (A2):

$$
\psi=\hat{R} \tilde{\psi}=\exp \left(-i \kappa \hat{x} \hat{\sigma}_{x} \int_{0}^{t} f(\tau) d \tau\right) \tilde{\psi}
$$

where $f(t)$ denotes the square function

$$
\begin{aligned}
& f(t)=1 \quad \text { for } \mathrm{t} \in[0, T / 4], \\
& f(t)=-1 \text { for } \mathrm{t} \in[T / 4, T / 2] .
\end{aligned}
$$

The transformed state satisfies the Schrödinger equation $i \partial_{t} \tilde{\psi}=\tilde{H}(t) \tilde{\psi}$, with the modified Hamiltonian

$$
\tilde{H}(t)=\hat{R}^{\dagger} \hat{H}_{0} \hat{R}=\frac{1}{2 m}\left\{\hat{p}_{y}^{2}+\left[\hat{p}_{x}-\kappa \hat{\sigma}_{x} \mathfrak{f}(t)\right]^{2}\right\}
$$

where $\mathfrak{f}(t)=\int_{0}^{t} f(\tau) d \tau$. Importantly, the result in Eq. (I3) is exact, and, in particular, it is valid for any $\omega$ or $\lambda_{R} \sim \kappa / \omega$. The evolution operator after half a period thus reads

$$
\hat{U}_{x}=e^{-i \int_{0}^{T / 2} \tilde{H}(t) d t}=e^{-i T\left[\hat{H}_{0} / 2-(\kappa T / 16 m) \hat{p}_{x} \hat{\sigma}_{x}\right]},
$$

which shows the fact that half of the XA sequence (55) has been treated exactly. The next subsequence $(t=T / 2 \rightarrow T)$ can be treated similarly, yielding the exact result

$$
\hat{U}_{y}=e^{-i T\left[\hat{H}_{0} / 2-(\kappa T / 16 m) \hat{p}_{y} \hat{\sigma}_{y}\right]}
$$

Finally, to lowest order in $T=2 \pi / \omega$, the Trotter expansion yields the Rashba SOC Hamiltonian

$$
\begin{aligned}
\hat{U}(T) & =\hat{U}_{y} \hat{U}_{x}=\exp \left(-i \hat{H}_{\mathrm{eff}}^{\mathcal{T}} T\right), \\
\hat{H}_{\mathrm{eff}}^{\mathcal{T}} & =\hat{H}_{0}-\lambda_{R} \hat{\boldsymbol{p}} \cdot \hat{\boldsymbol{\sigma}}+\mathcal{O}\left[\left(\Omega_{\mathrm{SO}} / \omega\right)^{2}\right],
\end{aligned}
$$

where $\lambda_{R}=\pi \kappa / 8 m \omega$, and where we introduce the small dimensionless parameter $\Omega_{\mathrm{SO}} / \omega$ with $\Omega_{\mathrm{SO}} \sim m \lambda_{R}^{2}$; here, we suppose that the momentum $p \sim m \lambda_{R}$ is of the order of the Rashba ring (i.e., the bottom of the Mexican hat).

\section{With the lattice}

A similar calculation allows us to evaluate the evolution operator for the XA sequence (55) defined on the lattice, namely, when substituting the operators in Eq. (55) by their lattice analogues (see Appendix H2).

As in the lattice-free case, we consider the unitary transformation in Eq. (I2). The transformed state satisfies the Schrödinger equation $i \partial_{t} \tilde{\psi}=\tilde{H}(t) \tilde{\psi}$, with the modified Hamiltonian

$\tilde{H}(t)=\frac{1}{2 m^{*}}\left\{\bar{p}_{y}^{2}+\bar{p}_{x}^{2} \cos [a \kappa \mathfrak{f}(t)]\right\}-\frac{1}{a m^{*}} \bar{p}_{x} \hat{\sigma}_{x} \sin [a \kappa \mathfrak{f}(t)]$,

where we use the formula (H6), and where the function $\mathfrak{f}(t)=\int_{0}^{t} f(\tau) d \tau$ was introduced in the last section. The evolution operator after half a period thus reads 


$$
\begin{aligned}
\bar{U}_{x} & =e^{-i \int_{0}^{T / 2} \tilde{H}(t) d t}=\exp \left(-i T \bar{H}_{x}\right), \\
\bar{H}_{x} & =\frac{\bar{p}_{y}^{2}}{4 m^{*}}+\frac{\bar{p}_{x}^{2}}{a \kappa T m^{*}} \sin [a \kappa T / 4]-\frac{4}{a^{2} \kappa T m^{*}} \bar{p}_{x} \hat{\sigma}_{x} \sin ^{2}[a \kappa T / 8] .
\end{aligned}
$$

A similar calculation can be performed for the next subsequence, yielding

$$
\begin{aligned}
\bar{U}_{y} & =\exp \left(-i T \hat{H}_{y}\right), \\
\bar{H}_{y} & =\frac{\bar{p}_{x}^{2}}{4 m^{*}}+\frac{\bar{p}_{y}^{2}}{a \kappa T m^{*}} \sin [a \kappa T / 4]-\frac{4}{a^{2} \kappa T m^{*}} \bar{p}_{y} \hat{\sigma}_{y} \sin ^{2}[a \kappa T / 8] .
\end{aligned}
$$

We point out that the expressions (I7) and (I8) are exact and that they constitute the direct lattice analogues of Eqs. (I4) and (I5). The Trotter expansion then yields the evolution operator after one period

$$
\begin{aligned}
\hat{U}(T)= & \bar{U}_{y} \bar{U}_{x}=\exp \left(-i \hat{H}_{\mathrm{eff}}^{\mathcal{T}} T\right), \\
\bar{H}_{\mathrm{eff}}^{\mathcal{T}}= & \frac{\bar{p}^{2}}{2 m^{*}}\left\{\frac{1}{2}+\frac{1}{2} \operatorname{sinc}\left(4 a m^{*} \lambda_{R}\right)\right\} \\
& +\overline{\boldsymbol{p}} \cdot \hat{\boldsymbol{\sigma}}\left\{\frac{\cos \left(4 a m^{*} \lambda_{R}\right)-1}{8\left(a m^{*}\right)^{2} \lambda_{R}}\right\},
\end{aligned}
$$

where $\lambda_{R}=\pi \kappa / 8 m^{*} \omega$ and $m^{*}=1 / 2 J a^{2}$ is the effective mass. Note that we recover exactly the result in Eq. (I6) for weak driving $\lambda_{R}<a J / 2$ and by taking the continuum limit. However, in the lattice framework, the maximum value of the effective Rashba SOC strength is limited. In particular, we find that this maximum value is obtained for $\lambda_{R}=\pi / 4 a m^{*}=a J(\pi / 2)$.

\section{APPENDIX J: EXACT TREATMENT OF THE $x y$ SCHEME}

In this Appendix, we derive the effective Hamiltonian in Eq. (66). We consider the $\alpha$-type $x y$ sequence [Eq. (51)]

$$
\left\{\frac{\bar{p}_{y}^{2}}{m}-\kappa \bar{x} \bar{\sigma}_{x}, \frac{\bar{p}_{y}^{2}}{m}-\kappa \bar{y} \bar{\sigma}_{y}, \frac{\bar{p}_{x}^{2}}{m}+\kappa \bar{x} \bar{\sigma}_{x}, \frac{\bar{p}_{x}^{2}}{m}+\kappa \bar{y} \bar{\sigma}_{y}\right\}
$$

and consider the special regime where $\kappa T=4 \pi / a$, that is, $\lambda_{R}=\pi \kappa / 8 m^{*} \omega=(\pi / 2) a J$. Furthermore, we consider that the system is set on a lattice, such that the operators in Eq. (J1) correspond to the lattice operators defined in Appendix H 2.

Here, in contrast with the analysis performed in Appendix I, we split the evolution operator $\bar{U}(T)$ corresponding to the sequence (J1) into its four primitive parts

$$
\begin{aligned}
\bar{U}(T)= & e^{-(i T / 4)\left(\bar{H}_{0}-\bar{B}\right)} e^{-(i T / 4)\left(\bar{H}_{0}-\hat{A}\right)} \\
& \times e^{-(i T / 4)\left(\bar{H}_{0}+\bar{B}\right)} e^{-(i T / 4)\left(\bar{H}_{0}+\bar{A}\right)} \\
= & \bar{U}_{-B} \bar{U}_{-A} \bar{U}_{+B} \bar{U}_{+A},
\end{aligned}
$$

and we analyze each operator $\bar{U}_{ \pm A, B}$ separately.

The operators $\bar{U}_{+A}$ and $\bar{U}_{-B}$ are readily simplified: Setting $\lambda_{R}=(\pi / 2) a J$, we directly obtain the factorized expressions

$$
\bar{U}_{+A}=e^{-i T \bar{p}_{y}^{2} / 4 m^{*}} e^{i \pi \bar{x} / a}, \quad \bar{U}_{-B}=e^{-i T \bar{p}_{x}^{2} / 4 m^{*}} e^{i \pi \bar{y} / a},
$$

where we use the simplification

$$
\exp \left(i \pi \bar{\mu} \bar{\sigma}_{\mu} / a\right)=\exp (i \pi \bar{\mu} / a), \quad \mu=x, y
$$

The latter is due to the fact that $\exp \left(i \pi m \hat{\sigma}_{x}\right)=(-1)^{m}$ for $m \in \mathbb{Z}$. The property (J4) is thus specific to the lattice operators $\bar{x} \bar{\sigma}_{x}$ and $\bar{y} \bar{\sigma}_{y}$ defined in Appendix H 2 .

The two other operators $\bar{U}_{-A}$ and $\bar{U}_{+B}$ require more care, as they are both of the form $\exp (X+Y)$ with $[X, Y] \neq 0$. The factorization can be performed through the Zassenhaus formula [77]

$$
e^{X+Y}=e^{X} e^{Y} e^{-(1 / 2)[X, Y]} e^{(1 / 3)[Y,[X, Y]]+(1 / 6)[X,[X, Y]]} \ldots .
$$

We find that the latter formula takes an elegant form when the operators satisfy the following cyclic relations:

$$
[X, Y]=Z, \quad[X, Z]=\mathfrak{a} Y, \quad[Y, Z]=0 .
$$

Indeed, under the conditions (J6), we find the exact result $e^{X+Y}=e^{X} \exp \{Y \sinh (\sqrt{\mathfrak{a}}) / \sqrt{\mathfrak{a}}-Z[-1+\cosh (\sqrt{\mathfrak{a}})] / \mathfrak{a}\}$.

Using the latter formula together with the cyclic conditions in Eq. (H5) yields

$\bar{U}_{-A}=e^{i \pi \bar{x} / a} e^{i T \lambda_{R}^{*} \bar{p}_{x} \bar{\sigma}_{x}}, \quad \bar{U}_{+B}=e^{i \pi \bar{y} / a} e^{-i T \lambda_{R}^{*} \bar{p}_{y} \bar{\sigma}_{y}}$,

where $\lambda_{R}^{*}=(2 / \pi) a J$ was introduced in Eq. (62).

We now have at our disposal factorized forms (J3)-(J7) for the four operators $\bar{U}_{ \pm A, B}$, which can be inserted into the evolution operator in Eq. (J2). Noting that

$$
\exp (-i \pi \bar{x} / a)\left(\bar{p}_{x} \bar{\sigma}_{x}\right) \exp (i \pi \bar{x} / a)=-\bar{p}_{x} \bar{\sigma}_{x},
$$

which directly results from Eq. (H6), we obtain the exact result

$$
\bar{U}(T)=e^{-i T \bar{p}_{x}^{2} / 4 m^{*}} e^{-i T \lambda_{R}^{*} \bar{p}_{x} \bar{\sigma}_{x}} e^{-i T \lambda_{R}^{*} \bar{p}_{y} \bar{\sigma}_{y}} e^{-i T \bar{p}_{y}^{2} / 4 m^{*}} .
$$

Applying the Trotter expansion to minimal order, we finally find the effective Rashba Hamiltonian announced in Eq. (66): 


$$
\begin{aligned}
\bar{U}(T) & =\bar{U}_{-B} \bar{U}_{-A} \bar{U}_{+B} \bar{U}_{+A}=\exp \left(-i \bar{H}_{\mathrm{eff}}^{\mathcal{T}} T\right) \\
\bar{H}_{\mathrm{eff}}^{\mathcal{T}} & =\frac{1}{2}\left(\frac{\bar{p}^{2}}{2 m^{*}}\right)+\lambda_{R}^{*} \overline{\boldsymbol{p}} \cdot \hat{\boldsymbol{\sigma}}+\mathcal{O}\left[\left(\Omega_{\mathrm{SO}} / \omega\right)^{2}\right]
\end{aligned}
$$

\section{APPENDIX K: AN ALTERNATIVE PERTURBATIVE APPROACH}

In this Appendix, we propose an alternative perturbative approach that is specifically dedicated to the general timedependent problem

$$
\hat{H}(t)=\hat{H}_{0}+\hat{V}(t)=\hat{H}_{0}+\hat{\mathcal{A}} f(t)+\omega \hat{\mathcal{B}} g(t),
$$

where the functions $f$ and $g$ are time periodic with a zero mean value over one period $T=2 \pi / \omega$. As motivated in the main text, the following method is particularly suited for problems in which $\hat{H}_{0} \sim \hat{\mathcal{A}} \sim \Omega \ll \omega$ and $\hat{\mathcal{B}} \sim 1$. Following the general approach of Appendix $C$, we write the effective Hamiltonian and kick operators as

$$
\hat{H}_{\mathrm{eff}}=e^{i \hat{K}(t)} \hat{H}(t) e^{-i \hat{K}(t)}+i\left(\frac{\partial e^{i \hat{K}(t)}}{\partial t}\right) e^{-i \hat{K}(t)}
$$

Since the time-dependent Hamiltonian in Eq. (K1) now contains a term that is proportional to the frequency $\omega$, the perturbative expansion in Eq. (C6) must be slightly modified to include a term $\hat{K}^{(0)}(t)$ :

$$
\hat{H}_{\text {eff }}=\sum_{n=0}^{\infty} \frac{1}{\omega^{n}} \hat{H}_{\text {eff }}^{(n)}, \quad \hat{K}(t)=\sum_{n=0}^{\infty} \frac{1}{\omega^{n}} \hat{K}^{(n)}(t) .
$$

Using the expansion formulas in Eqs. (C7) and (C8), we first identify the terms that are proportional to $\omega$ in Eq. (K2), which simply yields

$$
\hat{K}^{(0)}(t)=\hat{\mathcal{B}} G(t), \quad G(t)=\omega \int^{t} g(\tau) d \tau,
$$

where we impose that $G(t)$ should have a zero mean value over one period $\overline{G(t)}=(1 / T) \int_{0}^{T} G(t) d t=0$, as required for the kick operator in this formalism. Then, the first-order equation reads

$$
\begin{aligned}
\hat{H}_{\mathrm{eff}}^{(0)}= & \hat{H}_{0}+\hat{\mathcal{A}} f+i\left[\hat{K}^{(0)}, \hat{H}_{0}+\hat{\mathcal{A}} f\right] \\
& -\frac{1}{2}\left[\hat{K}^{(0)},\left[\hat{K}^{(0)}, \hat{H}_{0}+\hat{\mathcal{A}} f\right]\right] \\
& -\frac{i}{6}\left[\hat{K}^{(0)},\left[\hat{K}^{(0)},\left[\hat{K}^{(0)}, \hat{H}_{0}+\hat{\mathcal{A}} f\right]\right]\right] \\
& +\cdots+\hat{\mathcal{R}}\left(\hat{\mathcal{B}}, \hat{K}^{(1)}\right) \\
= & \exp (i G(t) \hat{\mathcal{B}})\left\{\hat{H}_{0}+\hat{\mathcal{A}} f(t)\right\} \exp (-i G(t) \hat{\mathcal{B}}) \\
& +\hat{\mathcal{R}}\left(\hat{\mathcal{B}}, \hat{K}^{(1)}\right),
\end{aligned}
$$

where the many terms grouped in the operator $\hat{\mathcal{R}}\left(\hat{\mathcal{B}}, \hat{K}^{(1)}\right)$ are used to define the next-order term $\hat{K}^{(1)}$, which will absorb the time-periodic terms with zero average. Taking the time average on both sides of Eq. (K5) finally yields

$$
\begin{aligned}
\hat{H}_{\text {eff }}= & \overline{\exp (i G(t) \hat{\mathcal{B}})\left\{\hat{H}_{0}+\hat{\mathcal{A}} f(t)\right\} \exp (-i G(t) \hat{\mathcal{B}})}+\mathcal{O}(1 / \omega), \\
= & \hat{H}_{0}+i \overline{G f}[\hat{\mathcal{B}}, \hat{\mathcal{A}}]-\frac{1}{2} \overline{G^{2}}\left[\hat{\mathcal{B}},\left[\hat{\mathcal{B}}, \hat{H}_{0}\right]\right] \\
& -\frac{1}{2} \overline{G^{2} f}[\hat{\mathcal{B}},[\hat{\mathcal{B}}, \hat{\mathcal{A}}]]-\frac{i}{6} \overline{G^{3}}\left[\hat{\mathcal{B}}\left[\hat{\mathcal{B}},\left[\hat{\mathcal{B}}, \hat{H}_{0}\right]\right]\right] \\
& -\frac{i}{6} \overline{G^{3} f}[\hat{\mathcal{B}}[\hat{\mathcal{B}},[\hat{\mathcal{B}}, \hat{\mathcal{A}}]]]+\cdots+\mathcal{O}(1 / \omega),
\end{aligned}
$$

which provides the effective Hamiltonian to lowest order in $1 / \omega$.

We now apply the expression in Eq. (K6) to the $\alpha$ pulse sequence introduced in Eq. (32):

$$
\alpha:\left\{\hat{H}_{0}+\hat{A}, \hat{H}_{0}+\hat{B}, \hat{H}_{0}-\hat{A}, \hat{H}_{0}-\hat{B}\right\} .
$$

The time-dependent Hamiltonian is of the form

$$
\hat{H}(t)=\hat{H}_{0}+\hat{V}(t)=\hat{H}_{0}+\hat{A} f(t)+\hat{B} g(t),
$$

where $f(t)$ and $g(t)$ are the square-wave functions associated with the sequence (K7). Comparing Eq. (K8) with the notations introduced in Eq. (K1), we note that $\hat{A}=\hat{A}$ and $\hat{\mathcal{B}}=\hat{B} / \omega$. Using the square-wave functions, we find

$$
\begin{array}{ll}
\overline{G f}=-\frac{\pi}{8}, & \overline{G^{2}}=\frac{\pi^{2}}{24}, \quad \overline{G^{2} f}=0, \\
\overline{G^{3}} & =0, \quad \overline{G^{3} f}=-\frac{\pi^{3}}{128} .
\end{array}
$$

Then, substituting $\hat{\mathcal{A}} \rightarrow \hat{A}$ and $\omega \hat{\mathcal{B}} \rightarrow \hat{B}$ into the formula (K6) reads

$$
\begin{aligned}
\hat{H}_{\mathrm{eff}}= & \hat{H}_{0}+\frac{i \pi}{8 \omega}[\hat{A}, \hat{B}] \\
& +\frac{\pi^{2}}{48 \omega^{2}}\left[\left[\hat{B}, \hat{H}_{0}\right], \hat{B}\right] \\
& +\frac{i \pi^{3}}{768 \omega^{3}}[[\hat{B},[\hat{A}, \hat{B}]], \hat{B}]+\cdots,
\end{aligned}
$$

which indeed provides the third-order term announced in Eq. (G4).

Finally, we consider the smooth single-harmonic driving

$$
\hat{H}(t)=\hat{H}_{0}+\hat{V}(t)=\hat{H}_{0}+\hat{A} \cos (\omega t)+\hat{B} \sin (\omega t),
$$

which approximates the $\alpha$ pulse sequence. In this case, $f(t)=\cos (\omega t)$ and $g(t)=\sin (\omega t)$, so that 


$$
\begin{array}{ll}
\overline{G f}=-\frac{1}{2}, & \overline{G^{2}}=\frac{1}{2}, \quad \overline{G^{2} f}=0, \\
\overline{G^{3}}=0, & \overline{G^{3} f}=-\frac{3}{8} .
\end{array}
$$

Then, substituting $\hat{\mathcal{A}} \rightarrow \hat{A}$ and $\omega \hat{\mathcal{B}} \rightarrow \hat{B}$ into the formula (K6) reads

$$
\begin{aligned}
\hat{H}_{\mathrm{eff}}= & \hat{H}_{0}+\frac{i}{2 \omega}[\hat{A}, \hat{B}] \\
& +\frac{1}{4 \omega^{2}}\left[\left[\hat{B}, \hat{H}_{0}\right], \hat{B}\right]+\frac{i}{16 \omega^{3}}[[\hat{B},[\hat{A}, \hat{B}]], \hat{B}]+\cdots,
\end{aligned}
$$

so that we partially recover the result in Eq. (G3). Indeed, the present method disregards the terms

$$
\begin{aligned}
& {\left[\left[\hat{A}, \hat{H}_{0}\right], \hat{A}\right], \quad\left[\hat{A},\left[\left[\hat{B}, \hat{H}_{0}\right], \hat{H}_{0}\right]\right],} \\
& {\left[\hat{B},\left[\left[\hat{A}, \hat{H}_{0}\right], \hat{H}_{0}\right]\right], \quad[[\hat{A},[\hat{A}, \hat{B}]], \hat{A}]}
\end{aligned}
$$

at this order of the computation, as it implicitly attributes different orders to the operators $\hat{H}_{0} \sim \hat{A}$ and $\hat{B} \sim \omega$; see Eq. (K1). Let us finally illustrate this last aspect based on the example presented in Sec. VIII A 1, which was based on the $\alpha$ sequence, and for which we found that the operators satisfied

$$
\hat{H}_{0} \sim \hat{A} \sim \Omega \sim \kappa / m \omega, \quad \hat{B} \sim \omega, \quad \Omega \ll \omega,
$$

according to the lowest Landau level characteristics. In this case, we indeed find that the terms identified by the present perturbative method and given in Eqs. (K9)-(K11) are all of the same order of $\Omega$, whereas the neglected terms in Eq. (K12) are all of the order $\Omega^{3} / \omega^{2} \ll \Omega$. In particular, this hierarchy illustrates the manner by which the present approach (potentially) allows us to partially resum the infinite series inherent to the formalism of Sec. II A, and hence, to guarantee the convergence of the perturbative expansion.

[1] X. L. Qi and S. C. Zhang, Topological Insulators and Superconductors, Rev. Mod. Phys. 83, 1057 (2011); M. Hasan and C. Kane, Colloquium: Topological Insulators, Rev. Mod. Phys. 82, 3045 (2010).

[2] P. A. Lee, N. Nagaosa, and X. G. Wen, Doping a Mott Insulator: Physics of High-Temperature Superconductivity, Rev. Mod. Phys. 78, 17 (2006).

[3] K. K. Gomes, W. Mar, W. Ko, F. Guinea, and H. C. Manoharan, Designer Dirac Fermions and Topological Phases in Molecular Graphene, Nature (London) 483, 306 (2012).

[4] M. Polini, F. Guinea, M. Lewenstein, H. C. Manoharan, and V. Pellegrini, Artificial Graphene as a Tunable Dirac Material, Nat. Nanotechnol. 8, 625 (2013).

[5] E. Kalesaki, C. Delerue, C. Morais Smith, W. Beugeling, G. Allan, and D. Vanmaekelbergh, Dirac Cones, Topological
Edge States, and Nontrivial Flat Bands in Two-Dimensional Semiconductors with a Honeycomb Nanogeometry, Phys. Rev. X 4, 011010 (2014).

[6] I. Carusotto and C. Ciuti, Quantum Fluids of Light, Rev. Mod. Phys. 85, 299 (2013).

[7] M. Hafezi, E. A. Demler, M. D. Lukin, and J. M. Taylor, Robust Optical Delay Lines with Topological Protection, Nat. Phys. 7, 907 (2011); M. Hafezi, S. Mittal, J. Fan, A. Migdall, and J. M. Taylor, Imaging Topological Edge States in Silicon Photonics, Nat. Photonics 7, 1001 (2013).

[8] M. C. Rechtsman, J. M. Zeuner, Y. Plotnik, Y. Lumer, D. Podolsky, F. Dreisow, S. Nolte, M. Segev, and A. Szameit, Photonic Floquet Topological Insulators, Nature (London) 496, 196 (2013).

[9] T. Jacqmin, I. Carusotto, I. Sagnes, M. Abbarchi, D. D. Solnyshkov, G. Malpuech, E. Galopin, A. Lemaitre, J. Bloch, and A. Amo, Direct Observation of Dirac Cones and a Flatband in a Honeycomb Lattice for Polaritons, Phys. Rev. Lett. 112, 116402 (2014).

[10] L. Tarruell, D. Greif, T. Uehlinger, G. Jotzu, and T. Esslinger, Creating, Moving and Merging Dirac Points with a Fermi Gas in a Tunable Honeycomb Lattice, Nature (London) 483, 302 (2012).

[11] J. Dalibard, F. Gerbier, G. Juzeliūnas, and P. Öhberg, Colloquium: Artificial Gauge Potentials for Neutral Atoms, Rev. Mod. Phys. 83, 1523 (2011).

[12] N. Goldman, G. Juzeliūnas, P. Öhberg, and I. B. Spielman, Light-Induced Gauge Fields for Ultracold Atoms, arXiv:1308.6533v1.

[13] H. Zhai, Spin-Orbit Coupled Quantum Gases, Int. J. Mod. Phys. B 26, 1230001 (2012); Degenerate Quantum Gases with Spin-Orbit Coupling, arXiv:1403.8021.

[14] M. A. H. Vozmediano, M. I. Katsnelson, and F. Guinea, Gauge Fields in Graphene, Phys. Rep. 496, 109 (2010); F. Guinea, M. I. Katsnelson, and A. K. Geim, Energy Gaps and a Zero-Field Quantum Hall Effect in Graphene by Strain Engineering, Nat. Phys. 6, 30 (2010); N. Levy, S. A. Burke, K. L. Meaker, M. Panlasigui, A. Zettl, F. Guinea, A. H. C. Neto, and M. F. Crommie, Strain-Induced Pseudomagnetic Fields Greater Than 300 Tesla in Graphene Nanobubbles, Science 329, 544 (2010); P. San-Jose, J. Gonzalez, and F. Guinea, Non-Abelian Gauge Potentials in Graphene Bilayers, Phys. Rev. Lett. 108, 216802 (2012).

[15] A. S. Sorensen, E. Demler, and M. D. Lukin, Fractional Quantum Hall States of Atoms in Optical Lattices, Phys. Rev. Lett. 94, 086803 (2005).

[16] L.-K. Lim, C. Morais Smith, and A. Hemmerich, StaggeredVortex Superfluid of Ultracold Bosons in an Optical Lattice, Phys. Rev. Lett. 100, 130402 (2008).

[17] A. Hemmerich, Effective Time-Independent Description of Optical Lattices with Periodic Driving, Phys. Rev. A 81, 063626 (2010).

[18] A. Eckardt, P. Hauke, P. Soltan-Panahi, C. Becker, K. Sengstock, and M. Lewenstein, Frustrated Quantum Antiferromagnetism with Ultracold Bosons in a Triangular Lattice, Europhys. Lett. 89, 10010 (2010).

[19] C. E. Creffield and F. Sols, Directed Transport in Driven Optical Lattices by Gauge Generation, Phys. Rev. A 84, 023630 (2011). 
[20] A. Bermudez, T. Schaetz, and D. Porras, Synthetic Gauge Fields for Vibrational Excitations of Trapped Ions, Phys. Rev. Lett. 107, 150501 (2011).

[21] A. R. Kolovsky, Creating Artificial Magnetic Fields for Cold Atoms by Photon-Assisted Tunneling, Europhys. Lett. 93, 20003 (2011); C. E. Creffield and F. Sols, Comment on "Creating Artificial Magnetic Fields for Cold Atoms by Photon-Assisted Tunneling by Kolovsky A. R., ” Europhys. Lett. 101, 40001 (2013).

[22] J. Struck, C. Ölschläger, M. Weinberg, P. Hauke, J. Simonet, A. Eckardt, M. Lewenstein, K. Sengstock, and P. Windpassinger, Tunable Gauge Potential for Neutral and Spinless Particles in Driven Optical Lattices, Phys. Rev. Lett. 108, 225304 (2012).

[23] P. Hauke et al., Non-Abelian Gauge Fields and Topological Insulators in Shaken Optical Lattices, Phys. Rev. Lett. 109, 145301 (2012).

[24] A. Bermudez, T. Schaetz, and D. Porras, Photon-AssistedTunneling Toolbox for Quantum Simulations in Ion Traps, New J. Phys. 14, 053049 (2012).

[25] B. M. Anderson, I. B. Spielman, and G. Juzeliunas, Magnetically Generated Spin-Orbit Coupling for Ultracold Atoms, Phys. Rev. Lett. 111, 125301 (2013).

[26] Zhi-Fang Xu, Li You, and M. Ueda, Atomic Spin-Orbit Coupling Synthesized with Magnetic-Field-Gradient Pulses, Phys. Rev. A 87, 063634 (2013).

[27] W. Zheng and H. Zhai, Floquet Topological States in Shaking Optical Lattices, arXiv:1402.4034v1.

[28] S. K. Baur, M. H. Schleier-Smith, and N. R. Cooper, Dynamic Optical Superlattices with Topological Bands, Phys. Rev. A 89, 051605(R) (2014).

[29] C. E. Creffield and F. Sols, Generation of Uniform Synthetic Magnetic Fields by Split Driving of an Optical Lattice, arXiv:1403.5915v1.

[30] D. R. Hofstadter, Energy Levels and Wave Functions of Bloch Electrons in Rational and Irrational Magnetic Fields, Phys. Rev. B 14, 2239 (1976).

[31] M. Aidelsburger, M. Atala, S. Nascimbène, S. Trotzky, Y.-A. Chen, and I. Bloch, Experimental Realization of Strong Effective Magnetic Fields in an Optical Lattice, Phys. Rev. Lett. 107, 255301 (2011).

[32] M. Aidelsburger, M. Atala, M. Lohse, J. T. Barreiro, B. Paredes, and I. Bloch, Realization of the Hofstadter Hamiltonian with Ultracold Atoms in Optical Lattices, Phys. Rev. Lett. 111, 185301 (2013).

[33] H. Miyake, G. A. Siviloglou, C. J. Kennedy, W. C. Burton, and W. Ketterle, Realizing the Harper Hamiltonian with Laser-Assisted Tunneling in Optical Lattices, Phys. Rev. Lett. 111, 185302 (2013).

[34] M. Aidelsburger, M. Lohse, C. Schweizer, M. Atala, J. T. Barreiro, S. Nascimbène, N. R. Cooper, I. Bloch, and N. Goldman, Revealing the Topology of Hofstadter Bands with Ultracold Bosonic Atoms, arXiv:1407.4205.

[35] J. Struck, C. Ölschläger, R. Le Targat, P. Soltan-Panahi, A. Eckardt, M. Lewenstein, P. Windpassinger, and K. Sengstock, Quantum Simulation of Frustrated Classical Magnetism in Triangular Optical Lattices, Science 333, 996 (2011).

[36] J. Struck, M. Weinberg, C. Ölschläger, P. Windpassinger, J. Simonet, K. Sengstock, R. Höppner, P. Hauke, A. Eckardt, M. Lewenstein, and L. Mathey, Engineering Ising- $X Y$
Spin-Models in a Triangular Lattice Using Tunable Artificial Gauge Fields, Nat. Phys. 9, 738 (2013).

[37] G. Jotzu, M. Messer, R. Desbuquois, M. Lebrat, T. Uehlinger, D. Greif, T. Esslinger, Experimental Realisation of the Topological Haldane Model, arXiv:1406.7874.

[38] F. D. M. Haldane, Model for a Quantum Hall Effect without Landau Levels: Condensed-Matter Realization of the Parity Anomaly, Phys. Rev. Lett. 61, 2015 (1988).

[39] J. Cayssol, B. Dóra, F. Simon, and R. Moessner, Floquet Topological Insulators, Phys. Status Solidi RRL 7, 101 (2013).

[40] N. H. Lindner, G. Refael, and V. Galitski, Floquet Topological Insulator in Semiconductor Quantum Wells, Nat. Phys. 7, 490 (2011).

[41] N. H. Lindner, D. L. Bergman, G. Refael, and V. Galitski, Topological Floquet Spectrum in Three Dimensions via a Two-Photon Resonance, Phys. Rev. B 87, 235131 (2013).

[42] H. L. Calvo, H. M. Pastawski, S. Roche, and L. E. F. Foa Torres, Tuning Laser-Induced Band Gaps in Graphene, Appl. Phys. Lett. 98, 232103 (2011).

[43] Y. H. Wang, H. Steinberg, P. Jarillo-Herrero, and N. Gedik, Observation of Floquet-Bloch States on the Surface of a Topological Insulator, Science 342, 453 (2013).

[44] P. Delplace, A. Gómez-León, and G. Platero, Merging of Dirac Points and Floquet Topological Transitions in ac-Driven Graphene, Phys. Rev. B 88, 245422 (2013).

[45] A. G. Grushin, A. Gómez-León, and T. Neupert, Floquet Fractional Chern Insulators, Phys. Rev. Lett. 112, 156801 (2014).

[46] A. Gómez-León, P. Delplace, and G. Platero, Engineering Quantum Anomalous Hall Plateaus and Anti-Chiral States with ac Fields, Phys. Rev. B 89, 205408 (2014).

[47] L. Jiang, T. Kitagawa, J. Alicea, A. R. Akhmerov, D. Pekker, G. Refael, J. Ignacio Cirac, E. Demler, M. D. Lukin, and P. Zoller, Majorana Fermions in Equilibrium and in Driven Cold-Atom Quantum Wires, Phys. Rev. Lett. 106, 220402 (2011).

[48] G. Liu, N. Hao, S.-L. Zhu, and W. M. Liu, Topological Superfluid Transition Induced by a Periodically Driven Optical Lattice, Phys. Rev. A 86, 013639 (2012).

[49] Q.-J. Tong, J.-H. An, J. Gong, H.-G. Luo, and C. H. Oh, Generating Many Majorana Modes via Periodic Driving: A Superconductor Model, Phys. Rev. B 87, 201109(R) (2013).

[50] M. Thakurathi, A. A. Patel, D. Sen, and A. Dutta, Floquet Generation of Majorana End Modes and Topological Invariants, Phys. Rev. B 88, 155133 (2013).

[51] Z.-B. Wang, H. Jiang, H. Liu, and X. C. Xie, Floquet Majorana Fermions in Driven Hexagonal Lattice Systems, arXiv:1404.3029.

[52] T. Kitagawa, E. Berg, M. Rudner, and E. Demler, Topological Characterization of Periodically Driven Quantum Systems, Phys. Rev. B 82, 235114 (2010).

[53] E. Suárez Morell and L. E. F. Foa Torres, Radiation Effects on the Electronic Properties of Bilayer Graphene, Phys. Rev. B 86, 125449 (2012).

[54] M. S. Rudner, N. H. Lindner, E. Berg, and M. Levin, Anomalous Edge States and the Bulk-Edge Correspondence for Periodically Driven Two-Dimensional Systems, Phys. Rev. X 3, 031005 (2013). 
[55] A. Gómez-León and G. Platero, Floquet-Bloch Theory and Topology in Periodically Driven Lattices, Phys. Rev. Lett. 110, 200403 (2013).

[56] M. Lababidi, I. I. Satija, and E. Zhao, Counter-propagating Edge Modes and Topological Phases of a Kicked Quantum Hall System, Phys. Rev. Lett. 112, 026805 (2014).

[57] P. M. Perez-Piskunow, G. Usaj, C. A. Balseiro, and L. E. F. Foa Torres, Floquet Chiral Edge States in Graphene, Phys. Rev. B 89, 121401(R) (2014).

[58] M. D. Reichl and E. J. Mueller, Floquet Edge States with Ultracold Atoms, arXiv:1404.3217.

[59] S. Rahav, I. Gilary, and S. Fishman, Effective Hamiltonians for Periodically Driven Systems, Phys. Rev. A 68, 013820 (2003).

[60] M. M. Maricq, Application of Average Hamiltonian Theory to the NMR of Solids, Phys. Rev. B 25, 6622 (1982).

[61] T. P. Grozdanov and M. J. Rakovic, Quantum System Driven by Rapidly Varying Periodic Perturbation, Phys. Rev. A 38, 1739 (1988).

[62] P. Avan, C. Cohen-Tannoudji, J. Dupont-Roc, and C. Fabre, Effect of High Frequency Irradiation on the Dynamical Properties of Weakly Bound Electrons, J. Phys. (Paris) 37, 993 (1976).

[63] C. F. Foot, Atomic Physics (Oxford University Press, New York, 2005).

[64] A. Eckardt, T. Jinasundera, C. Weiss, and M. Holthaus, Analog of Photon-Assisted Tunneling in a Bose-Einstein Condensate, Phys. Rev. Lett. 95, 200401 (2005).

[65] H. Lignier, C. Sias, D. Ciampini, Y. Singh, A. Zenesini, O. Morsch, and E. Arimondo, Dynamical Control of MatterWave Tunneling in Periodic Potentials, Phys. Rev. Lett. 99, 220403 (2007).

[66] A. Eckardt and M. Holthaus, ac-Induced Superfluidity, Europhys. Lett. 80, 50004 (2007).

[67] A. Eckardt, M. Holthaus, H. Lignier, A. Zenesini, D. Ciampini, O. Morsch, and E. Arimondo, Exploring Dynamic Localization with a Bose-Einstein Condensate, Phys. Rev. A 79, 013611 (2009).

[68] K. Kudo and T.S. Monteiro, Theoretical Analysis of Super-Bloch Oscillations, Phys. Rev. A 83, 053627 (2011).

[69] D. Jaksch and P. Zoller, Creation of Effective Magnetic Fields in Optical Lattices: The Hofstadter Butterfly for Cold Neutral Atoms, New J. Phys. 5, 56 (2003).

[70] F. Gerbier and J. Dalibard, Gauge Fields for Ultracold Atoms in Optical Superlattices, New J. Phys. 12, 033007 (2010).

[71] F. Guinea, Spin-Orbit Coupling in a Graphene Bilayer and in Graphite, New J. Phys. 12, 083063 (2010).

[72] C. L. Kane and E. J. Mele, Quantum Spin Hall Effect in Graphene, Phys. Rev. Lett. 95, 226801 (2005).

[73] J. D. Sau, R. Sensarma, S. Powell, I. B. Spielman, and S. Das Sarma, Chiral Rashba Spin Textures in Ultracold Fermi Gases, Phys. Rev. B 83, 140510(R) (2011).
[74] X.-L. Qi, Y.-S. Wu, and S.-C. Zhang, Topological Quantization of the Spin Hall Effect in Two-Dimensional Paramagnetic Semiconductors, Phys. Rev. B 74, 085308 (2006).

[75] W. Beugeling, N. Goldman, and C. Morais Smith, Topological Phases in a Two-Dimensional Lattice: Magnetic Field versus Spin-Orbit Coupling, Phys. Rev. B 86, 075118 (2012).

[76] Z. Qiao, S. A. Yang, W. Feng, W.-K. Tse, J. Ding, Y. Yao, J. Wang, and Q. Niu, Quantum Anomalous Hall Effect in Graphene from Rashba and Exchange Effects, Phys. Rev. B 82, 161414(R) (2010).

[77] F. Casas, A. Murua, and M. Nadinic, Efficient Computation of the Zassenhaus Formula, Comput. Phys. Commun. 183, 2386 (2012).

[78] N. R. Cooper (private communication).

[79] A. Lazarides, A. Das, and R. Moessner, Periodic Thermodynamics of Isolated Quantum Systems, Phys. Rev. Lett. 112, 150401 (2014).

[80] M. Langemeyer and M. Holthaus, Energy Flow in Periodic Thermodynamics, Phys. Rev. E 89, 012101 (2014).

[81] L. D’Alessio and A. Polkovnikov, Many-Body Energy Localization Transition in Periodically Driven Systems, Ann. Phys. (Amsterdam) 333, 19 (2013).

[82] S. Choudhury and E. J. Mueller, Stability of a Floquet BoseEinstein Condensate in a One-Dimensional Optical Lattice, arXiv:1405.1398.

[83] M. Esposito, U. Harbola, and S. Mukamel, Nonequilibrium Fluctuations, Fluctuation Theorems, and Counting Statistics in Quantum Systems, Rev. Mod. Phys. 81, 1665 (2009).

[84] O. Gamel and D. F. V. James, Time-Averaged Quantum Dynamics and the Validity of the Effective Hamiltonian Model, Phys. Rev. A 82, 052106 (2010).

[85] A. Rapp, X. Deng, and L. Santos, Ultracold Lattice Gases with Periodically Modulated Interactions, Phys. Rev. Lett. 109, 203005 (2012).

[86] M. Di Liberto, C. E. Creffield, G. I. Japaridze, and C. Morais Smith, Quantum Simulation of Correlated-Hopping Models with Fermions in Optical Lattices, Phys. Rev. A 89, 013624 (2014).

[87] H. M. Price and N. R. Cooper, Mapping the Berry Curvature from Semiclassical Dynamics in Optical Lattices, Phys. Rev. A 85, 033620 (2012).

[88] D. A. Abanin, T. Kitagawa, I. Bloch, and E. Demler, Interferometric Approach to Measuring Band Topology in 2D Optical Lattices, Phys. Rev. Lett. 110, 165304 (2013).

[89] A. Dauphin and N. Goldman, Extracting the Chern Number from the Dynamics of a Fermi Gas: Implementing a Quantum Hall Bar for Cold Atoms, Phys. Rev. Lett. 111, 135302 (2013).

[90] D. Xiao, M. C. Chang, and Q. Niu, Berry Phase Effects on Electronic Properties, Rev. Mod. Phys. 82, 1959 (2010).

[91] I. S. Gradshteyn and I. M. Ryzhik, Table of Integrals, Series, and Products (Academic, New York, 2007) 7th ed. 